\title{
A Review of the Active Management of Norway's Government Pension Fund Global
}

Citation for published version (APA):

Bauer, R., Christiansen, C., \& Doskeland, T. (2022). A Review of the Active Management of Norway's Government Pension Fund Global. Norwegian Ministry of Finance.

Document status and date:

Published: 03/01/2022

Document Version:

Publisher's PDF, also known as Version of record

\section{Please check the document version of this publication:}

- A submitted manuscript is the version of the article upon submission and before peer-review. There can be important differences between the submitted version and the official published version of record.

People interested in the research are advised to contact the author for the final version of the publication, or visit the DOI to the publisher's website.

- The final author version and the galley proof are versions of the publication after peer review.

- The final published version features the final layout of the paper including the volume, issue and page numbers.

Link to publication

\footnotetext{
General rights rights.

- You may freely distribute the URL identifying the publication in the public portal. please follow below link for the End User Agreement:

www.umlib.nl/taverne-license

Take down policy

If you believe that this document breaches copyright please contact us at:

repository@maastrichtuniversity.nl

providing details and we will investigate your claim.
}

Copyright and moral rights for the publications made accessible in the public portal are retained by the authors and/or other copyright owners and it is a condition of accessing publications that users recognise and abide by the legal requirements associated with these

- Users may download and print one copy of any publication from the public portal for the purpose of private study or research.

- You may not further distribute the material or use it for any profit-making activity or commercial gain

If the publication is distributed under the terms of Article $25 \mathrm{fa}$ of the Dutch Copyright Act, indicated by the "Taverne" license above, 


\title{
A Review of the Active Management of Norway's Government Pension Fund Global
}

\author{
Rob Bauer ${ }^{1}$, Charlotte Christiansen $^{2}$, and Trond Døskeland ${ }^{3}$ \\ ${ }^{1}$ Department of Finance, Maastricht University School of Business and Economics, Netherlands, \\ Email: r.bauer@maastrichtuniversity.nl \\ ${ }^{2}$ CREATES, Department of Economics and Business Economics, Aarhus University, Denmark \\ Email: cchristiansen@econ.au.dk \\ ${ }^{3}$ Department of Business and Management Science, NHH Norwegian School of Economics, Norway, \\ Email: trond.doskeland@nhh.no
}

January 3, 2022 


\section{Acknowledgments}

We have received helpful comments from the Ministry of Finance (MoF) and Norges Bank Investment Management (NBIM). We circulated a previous version (dated December 6, 2021) of this report to NBIM, the MoF, and their expert panel (Gerlof de Vrij and Sung Cheng Chih). We presented that version to the MoF and their expert panel on December 13, 2021. We have also benefited from several meetings with NBIM during the work on the report and received helpful input from various external stakeholders: B. Espen Eckbo, Espen Henriksen, Halvor Hoddevik, Knut Kjær, Richard Priestley, Espen Sirnes, and Karin Thorburn. We thank PhD candidate André Wattø Sjuve from NHH for replicating our calculations and John Morris (Editide) for excellent proofreading.

The views expressed in this report are solely the responsibility of the authors. 


\section{Executive summary}

In this review, we evaluate the active management by Norges Bank Investment Management (NBIM) of the Norwegian Government Pension Fund Global (the Fund). In this executive summary, we provide an overview of the main observations and suggestions. More details on the quantitative and qualitative analysis, the methodology, and our observations and suggestions can be found in the full version of the report.

\section{Introduction to the Fund}

The Norwegian government founded the Fund in 1986 to accumulate surplus from the Norwegian oil revenues, and capital injections into the Fund started in 1996. The Fund's assets under management (AUM) have grown steadily since then due to new capital injections, but predominantly due to high financial returns on the Fund's investments.

Initially, the Fund's investments were in government fixed-income securities. In January 1998, the Fund began to invest in equity, starting with a target portfolio of $40 \%$ equity and $60 \%$ fixed income. The allocation to equity has continued to grow. Today, the target is $70 \%$. In 2010 , the fund added unlisted real estate to the portfolio. In the summer of 2021, NBIM made its first renewable-infrastructure investments.

In most of the analysis, we use data from January 1998 through September 2021. As requested in the mandate, we additionally examine the most recent period (January 2017 to September 2021). We use USD returns for our calculations. For the analysis in monetary terms, we use the AUM measured in NOK. This review is the first to study in detail the investment strategies within asset classes (substrategies). For these subportfolios, the sample period is shorter (January 2013 to September 2021).

The Ministry of Finance (MoF) designed and provided the benchmark for the overall performance evaluation of the Fund. In our substrategy performance evaluation, we also use the internal benchmarks provided by NBIM.

\section{Performance evaluation}

In our evaluation of the Fund's performance, we first consider the performance evaluation without any risk adjustments. To this end, we use the mean active returns and value added of the Fund and its various strategies. We integrate management costs in this analysis and show gross and net active returns and value added.

Since 1998, the Fund's annualized average net total return has been close to seven percent (7.03\%). Since the benchmark has shown an annualized return of $6.83 \%$, the average net active annualized return at the total Fund level is positive, but small: $0.20 \%$. However, our analysis also shows that the corresponding $p$-value is high (.36), implying that the documented active returns at the total Fund level are not very likely to be different from zero. The Fund resembles an index fund in that its returns are close to its benchmark.

At the total equity level, the annualized mean active returns are $0.36 \%$ with a $p$-value of .06 . In this setting without risk adjustment, equity portfolios significantly create value before costs: Gross active 
returns are $0.47 \%$ ( $p$-value of .01). At the fixed-income management level, net (gross) active returns are smaller: $0.13 \%(0.17 \%)$ with very high $p$-values. We are hesitant to draw any conclusions about the real-estate portfolio as we have reservations on the benchmarking process and data properties. The total accumulated value added for the Fund for the full sample period is NOK 228 billion before and NOK 170 billion after costs. Furthermore, we find that a large part of the value added for the total portfolio can be attributed to active management in equity.

We find some evidence of value-adding equity strategies. Security selection strategies, net of costs, show positive active returns: $1.27 \%$ with a $p$-value of .04 . The break-up into internal and externalsecurity-selection strategies shows that this positive result is likely caused by the net positive active return of external management strategies (2.15\% with a $p$-value of .00). Although we document a positive net active return of $0.93 \%$ for internal-security-selection strategies, again before risk-adjustment, the corresponding $p$-value (.20) shows that this outperformance is not statistically significant. The enhanced indexing investment strategies (asset positioning and security lending) provide small (in total $0.20 \%$ ) but consistently positive average active returns (with $p$-values close to zero). The value added from these strategies is comparable to that from security selection due to a large capital base. The third equity substrategy, fund allocation, is very difficult to assess as it is an amalgam of many diverse strategies. Nonetheless, it is safe to conclude that equity-fund allocation strategies do not provide any positive active returns.

Inspection of the fixed-income strategies shows a similar picture for enhanced indexing strategies (asset positioning and security lending). Net active returns are positive $(0.28 \%)$, and $p$-values are again close to zero. However, net active returns of security selection and allocation strategies do not appear significantly different from zero (given the high $p$-values of .24 and .11, respectively). Although we report the results for the listed and unlisted-real-estate substrategies, we are again very cautious about drawing any strong conclusions.

It is a sheer impossible task to choose a risk model that encompasses the Fund's full set of investment strategies and substrategies and the underlying historical dynamics (such as recent benchmark changes) in the period under investigation. The dynamic nature of the investment and benchmarking process would also require a risk-adjustment procedure able to capture time-varying factor exposures. We feel that this would not reduce but increase complexity. Therefore, it would not contribute positively to the accountability of NBIM to the MoF and the Norwegian public.

For this reason, we use the standard capital asset pricing model (CAPM) as the base case for risk adjustment. Subsequently, we extend this with a well-known and widely applied version of the Fama and French (2015) factor model (the five-factor model extended with two additional factors for fixed income), also advocated by Dahlquist, Polk, Priestley and Ødegaard (2015). This method gives us preliminary quantitative insights, but we are aware of potential model and parameter uncertainty that comes with these performance evaluation models.

Our key conclusion based on risk-adjusted results is that alphas from these regressions are very small at the total Fund level (for the full sample period): $0.06 \%$ with high $p$-values. Compared to the basic performance comparison with the benchmark (active returns), risk-adjusted alphas are now somewhat smaller at the equity-management level: $0.25 \%$ (for both the CAPM and the factor model) with $p$-values larger than .10. At the fixed-income-management level, alphas are again small and not 
significantly different from zero. We observe, though, that alphas are generally higher in the recent sample period (2017-2021).

The risk-adjusted alphas for substrategies are generally very close to the average active returns in the basic performance evaluation without risk adjustment. External security selection and enhanced indexing strategies (asset positioning and security lending) for equity and fixed income have alphas significantly different from zero. We also find that the mean active return of the equity internal security-selection strategy decreases considerably after risk adjustment.

Interestingly, we also find that NBIM does not make full use of its ex-ante tracking-error limit of $1.25 \%$. In the full sample period, the ex-post tracking error at the total Fund level is $0.64 \%$. In the last five years, the ex-post tracking error has declined to a very low level: $0.32 \%$.

\section{A complex structure}

Despite its low active risk profile, NBIM's organization runs more than 200 investment strategies and substrategies. As a review committee, we are the first to provide detailed insights into key substrategies. However, providing a full overview of all substrategies is not possible; the time commitment and resources that come with the review committee's mandate do not allow us to investigate them all in detail.

NBIM has been very helpful in delivering information and providing background on their investments. Moreover, we feel that NBIM reports transparently on its investments to the public. Nonetheless, it has been quite a challenge for us to understand NBIM's investment organization in granular detail, its key investment strategies and substrategies, and the cost allocation to the strategies and substrategies.

It is not always clear to us how the performance information NBIM reports to the public can be mapped to the Fund's investment departments and portfolio-management teams. This makes it very difficult for outsiders to discern which (sub)strategies performed well and, importantly, what can be learned from this for the future.

In our view, the observed complexity reduces outside stakeholders' opportunity to provide meaningful feedback to NBIM. In addition, as previous reviews have documented, we do not have full clarity on how internal benchmarks are constructed for substrategies. This lack of clarity will impact the possibility of meaningfully reviewing substrategies.

Given the observed complexity and many other confounding factors that can be found in the full report, we feel that we must be cautious about giving too much weight to any individual numbers presented.

\section{Potential conflicts of interest in NBIM's mandate}

The total returns delivered by the Fund are largely determined by the choice of the strategic benchmark determined by the MoF. Since its inception in 1998, the annualized average net total return of the Fund is around seven percent, whereas the average net active return is around $0.20 \%$. These numbers show the relative importance of the strategic decision-making process.

NBIM's organization understandably seems to be focused on the key elements of the MoF's mandate: to deliver as large an active return as possible in predominantly public markets within the mandate's risk limit. The mandate also states that NBIM should provide advice to the MoF in matters of 
the Fund's strategic direction. However, the strategic investment direction (including strategic asset allocation) is not determined by NBIM.

Updates to the mandate and resulting benchmark changes will occur from time to time. Good examples are the inclusion and subsequent removal of real estate and emerging-markets fixed income in the benchmark. Changes that involve starting up or winding down illiquid investments involve complexity, organizational impact, and high trading costs.

For example, human capital had been attracted to take on the management of the unlisted-realestate portfolio. However, with the formal removal of real estate from the benchmark in 2017, the MoF indirectly signaled that NBIM will not have the possibility to build up a sizable, long-term stake in unlisted real estate. From that moment onwards, the Fund's exposure to real-estate operations was bound by the mandate's tracking error. This indirect signaling may have a substantial impact on NBIM's future ability to attract and retain key personnel in the context of the unlisted-real-estate and infrastructure teams.

Investing in certain private or complex asset categories, which are not part of the Fund's benchmark, also comes with another major challenge. We illustrate this using the real-estate context. Despite the removal of real estate from the benchmark in 2017, NBIM is allowed to continue investing in unlisted and listed real estate. These investments would be interpreted as active deviations from the mandate's benchmark. Going forward, these investments would compete for parts of the active risk budget with other investment strategies in the Fund. In our conversations with NBIM, we were regularly told that real estate, despite not being part of the benchmark, retains a diversification role.

In our view, this context gives rise to potential future conflicts of interest and inefficiencies in a few dimensions. NBIM, in fact, has two objectives. One is to invest in real estate as a diversifying asset (in the total return and risk space) and the other is to develop real-estate investment strategies as a source of active return versus the mandate's benchmark (in the active return and risk space). Implicitly, this means that the Fund has two key objectives: achieving the highest possible Sharpe ratio and achieving the highest possible information ratio $(I R)$. However, the Fund has just one instrument: the real-estate investment portfolio. This construct seems to be at odds with the Tinbergen rule (transferred from macroeconomics to investments and paraphrased): For each objective, one needs to have one instrument (see Tinbergen, 1952). These conflicting objectives could lead to organizational challenges. From a Sharpe-ratio perspective, it may be wise to continue to invest in real estate, but from an IR perspective, it may be wise to deploy the organizational focus and tracking-error budget on other value-adding strategies.

\section{Active ownership and responsible investments}

In the full report, we also address another question: Does NBIM's mandate regarding active ownership strategies impact the Fund's potential (positive and negative) to provide active returns? Increased and intensified (future) efforts in active ownership strategies, as well as more explicit integration of sustainability-related information into the portfolio-management process, may impact NBIM's potential to create value with active investment strategies. These activities may require a different focus in the investment strategy and, as such, could compete with resources currently deployed in other active investment strategies. 
NBIM's current activities in the field of responsible investments are well documented in its 2020 responsible-investments report (NBIM, 2020j). This report shows that NBIM takes its role as a responsible-asset owner very seriously. NBIM also states in a letter to the MoF (July 2021) that their portfolio managers consider climate risk and climate-related investment opportunities before deciding to invest in individual companies. The MoF's climate-risk expert group expects that NBIM can test and potentially influence the robustness of business models of the companies in which NBIM has invested (see Skancke, Halvorsen, Hanstad and Thornburn, 2021). Active ownership may also contribute to companies channeling more capital to profitable projects in the transition to a low-carbon economy. Active ownership may, according to the expert group, also strengthen financial markets' ability to price climate risks, and they add that failed engagement may eventually lead to divestment from companies.

We analyze how an increased focus on active-ownership strategies could impact NBIM's potential to achieve active returns. First, we discuss how active management and active-ownership strategies may have different objectives, which can lead to conflicts of interest. Second, we discuss the conditions for a successful implementation of active-ownership strategies.

First, targeting companies through active-ownership strategies (engagement, voting, threat of divestments, and more) has the objective to help companies perform better and, as such, is targeted at total returns (in contrast to targeting active returns). Successfully engaging with a company will increase the return of the Fund's portfolio (ceteris paribus), but the active return versus the benchmark will be negatively affected if the company's portfolio weight versus the benchmark weight is negative.

The objective to intensify and extend the active-ownership effort may be at odds with the objective to harvest active returns. It also raises a question: Who decides which objective is to be prioritized in which context? This is another example of having one instrument (the Fund's investment portfolio) and two objectives (active-ownership impact and active return).

The climate-risk expert group's statement that NBIM should be guided by international climate agreements to which Norway has acceded, raises another source of potential conflicts. NBIM's mandate should give guidance on which decisions are to be prioritized. Note that this is again an example of having two objectives (zero emission targets and active returns) and just one instrument (the portfolio).

Second, certain conditions must be met to successfully extend and intensify active-ownership strategies. This could imply deploying many additional resources. This development will materially affect NBIM's budget. If the required budget must be partially financed by redeploying resources in the current budget, it may affect resources spent on other (active-management) activities.

Credibly, effectively, and successfully engaging with company management on material ESG (environmental, social, and governance) issues requires the ability to engage in conversations with company management, sector-specific knowledge of business models, sector-specific and beyond knowledge of ESG material issues, and knowledge of active-ownership strategy tools. This shows that the execution of these strategies requires an investment in or redeployment of human capital. In our view, it is an advantage that NBIM has developed expertise in engaging with companies. The combined knowledge and expertise of the governance team and the internal security-selection teams ensure credible access to company management. We are not sure, though, whether the task related to increased and intensified active ownership in the whole portfolio, as proposed by the climate-risk expert group, can be executed with the current team's capacity and background. 
Moreover, active-ownership strategies require a long-horizon mindset and a greater focus than before on material issues in the ESG space. The same logic holds for an increased emphasis on adequate climate-risk reporting by companies.

In our view, NBIM's leadership team has an important task ahead of it in creating a culture that contributes to successful active-ownership strategies while at the same time running the investment portfolios. That requires clarity on NBIM's mandate regarding ownership strategies going forward. When the MoF decides how to integrate the climate-risk expert group's recommendations into the mandate, it should be clear how the different objectives are resourced and prioritized, which targets should be achieved, and how incentive schemes for key decision makers are compatible with these priorities.

\section{Our key suggestions}

Given our performance analysis and qualitative assessments in the full report, we offer five key suggestions.

- We encourage the MoF to provide NBIM with a mandate with a clear active-return target. The target could serve as guidance for NBIM's strategic decision making regarding its active management. Further, it would give future review committees a better basis for their feedback. We further urge the MoF to investigate why NBIM does not take full advantage of its trackingerror limit. The objective of this study would be to understand why this ex-ante limit is not reached, whether any operational impediments or structural barriers play a role, and whether and how they can be lifted, if relevant.

- We suggest that NBIM improves the communication with external stakeholders on the Fund's active strategies and substrategies, giving access to more granular information on the internal benchmarking process and the cost allocation at the level of strategies, substrategies, and subsubstrategies. This may also involve providing more background on the active-risk appetite.

- We encourage the MoF to reduce the role of factor models in evaluating the Fund's performance and, instead, put more focus and emphasis on the qualitative assessments of the Fund's organization that investigate such modern aspects as governance quality, long-term mindset, and creating the appropriate culture for a successful execution of the strategy. We advise future review committees to evaluate NBIM's active management with simple, understandable, and widely accepted performance-evaluation models.

- If the MoF confirms the removal of real estate from NBIM's benchmark, we seriously doubt the long-term viability of NBIM managing unlisted-real-estate investments. If unlisted real estate is deemed important for diversification purposes by the MoF, we advise restoring real estate to the benchmark.

- We urge the MoF to provide clarity in the mandate on the objectives and prioritization of active ownership strategies, as well as on what parts of this prioritization are NBIM's purview versus which are prescribed in the mandate. 


\section{Contents}

1 Introduction $\quad 11$

1.1 Roadmap of the review . . . . . . . . . . . . . . . . . . . 11

1.2 Performance-evaluation measures . . . . . . . . . . . . . . . . . . . 13

1.3 Literature . . . . . . . . . . . . . . . . . . . . . . . . 14

2 Overview of the Fund $\quad 18$

2.1 Data . . . . . . . . . . . . . . . . . . . . . . . 19

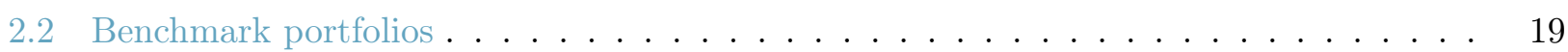

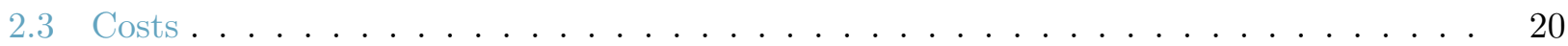

2.4 Realized total return and risk . . . . . . . . . . . . . . . . . . 22

3 Fund portfolio value creation $\quad 25$

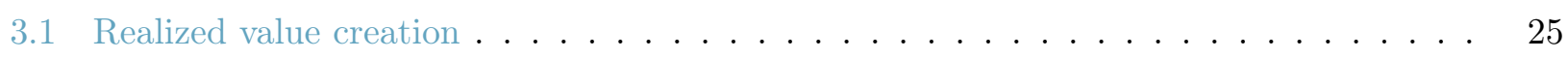

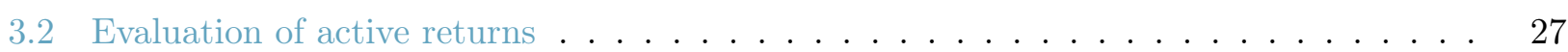

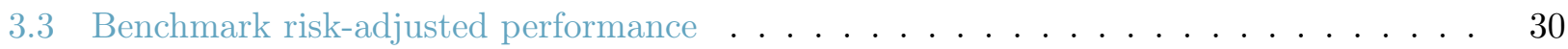

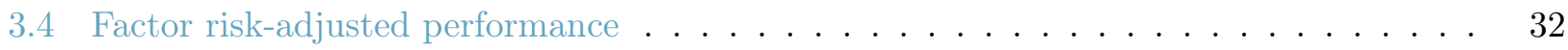

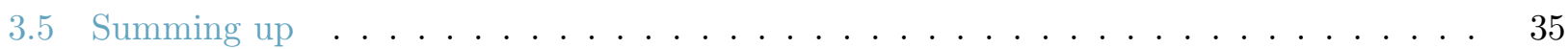

4 Equity value creation $\quad \mathbf{3 7}$

4.1 Realized value creation . . . . . . . . . . . . . . . . . . 37

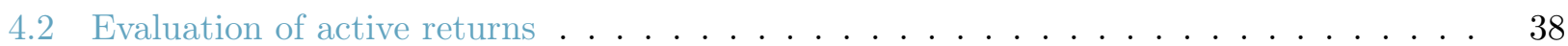

4.3 Benchmark risk-adjusted performance . . . . . . . . . . . . . . . 39

4.4 Factor risk-adjusted performance . . . . . . . . . . . . . . . . . 40

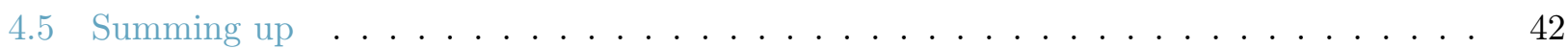

$5 \quad$ Equity strategies $\quad 43$

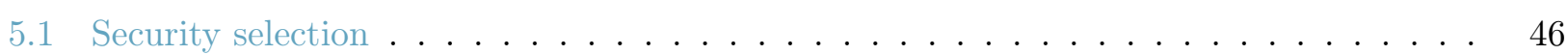

5.2 Enhanced indexing . . . . . . . . . . . . . . . . . . 49

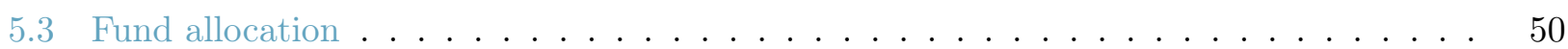

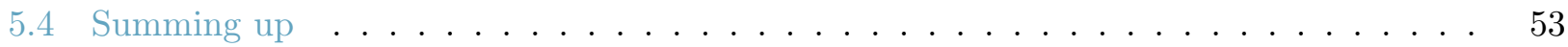

$6 \quad$ Fixed-income value creation $\quad \mathbf{5 4}$

6.1 Realized value creation . . . . . . . . . . . . . . . . . . . 54

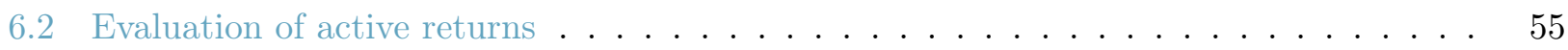

6.3 Benchmark risk-adjusted performance . . . . . . . . . . . . . . 57

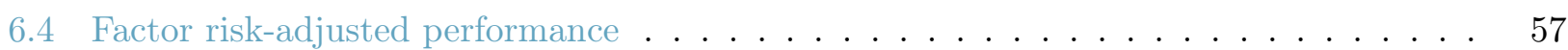

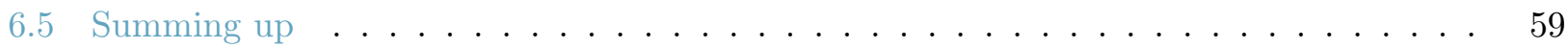

$\begin{array}{lll}7 & \text { Fixed-income strategies } & \mathbf{6 0}\end{array}$

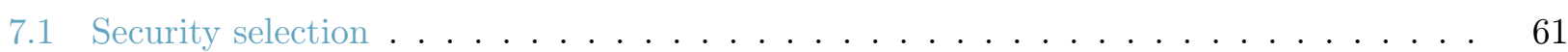

7.2 Enhanced indexing . . . . . . . . . . . . . . . . . 62

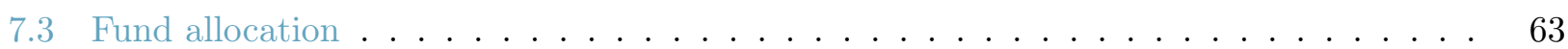




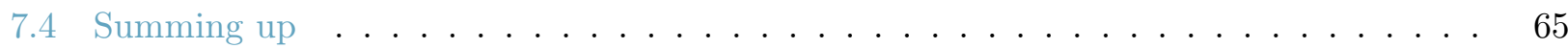

8 Real-estate value creation $\quad \mathbf{6 6}$

8.1 Real-estate portfolio . . . . . . . . . . . . . . . . . . 66

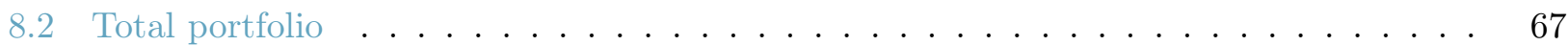

8.3 Unlisted portfolio . . . . . . . . . . . . . . . . . . . . . . 68

8.4 Listed portfolio . . . . . . . . . . . . . . . . . . . . . . . . 69

8.5 Financing model for real-estate portfolio . . . . . . . . . . . . . . . . . 69

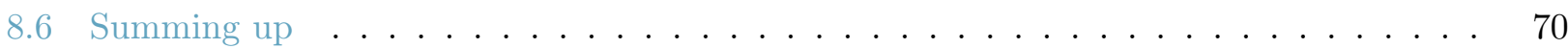

9 Potential for value creation: Observations and suggestions $\quad 72$

9.1 Evaluation of value creation . . . . . . . . . . . . . . . . . . . 72

9.2 Risk-adjusted performance evaluation . . . . . . . . . . . . . . . . 75

9.3 A complex structure . . . . . . . . . . . . . . . . . . . 77

9.4 Potential conflicts of interest in NBIM's mandate . . . . . . . . . . . . . . . 79

9.5 Active ownership and responsible investments . . . . . . . . . . . . . . 82

10 Concluding comments $\quad \mathbf{8 7}$

$\begin{array}{lr}\text { References } & \mathbf{8 9}\end{array}$

$\begin{array}{lr}\text { Appendices } & \mathbf{9 6}\end{array}$

$\begin{array}{lr}\text { A Mandate } & \mathbf{9 6}\end{array}$

$\begin{array}{ll}\text { B Data } & 97\end{array}$

$\begin{array}{lr}\text { C Appendix for Section } 2 & \mathbf{9 8}\end{array}$

$\begin{array}{ll}\text { D Appendix for Section 3 } & 100\end{array}$

$\begin{array}{lll}\text { E } & \text { Appendix for Section } 4 & 101\end{array}$

F Appendix for Section 5 103

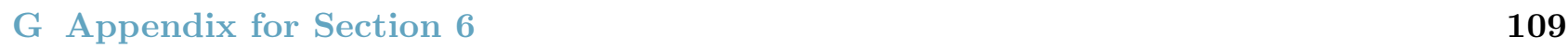

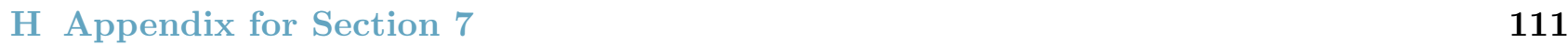

I Appendix for Section 8 $\quad 116$ 


\section{Introduction}

The Norwegian Government Pension Fund Global (the Fund) is a large sovereign wealth fund owned by the Norwegian people. Norway's Ministry of Finance (MoF) has issued a mandate that defines the Fund's targeted asset allocation, active risk limits, and other key attributes (see Ministry of Finance, 2021). Norges Bank Executive Board is formally responsible for the mandate. One of its departments, Norges Bank Investment Management (NBIM), implements the mandate on a day-to-day basis. Chambers, Dimson and Ilmanen (2012, 2021) characterize the investment strategy as the Norway model, an asset-oriented model with investments in traditional liquid assets (see Exhibit 3 in Chambers et al., 2021). Given the moderate ex-ante tracking-error limit (1.25\%) and the low ex-post tracking error we document, the Fund can, to a large extent, be viewed as an (enhanced) index fund.

\section{$1.1 \quad$ Roadmap of the review}

Figure 1 shows the governance structure of the Fund's management. The Norwegian people, represented by parliament and related bodies, can be viewed as the asset owner whose risk appetite, investment beliefs, and preferences determine the Fund's long-term strategy. The MoF sets a mandate consistent with the Fund's strategy that serves as the basis for NBIM's asset-management activities. Every four years, the MoF installs a review committee whose main task is to evaluate NBIM's active-management strategies. The red shaded background in Figure 1 shows the focus area of the active-management review. The complete review committee's mandate is available in Appendix A.

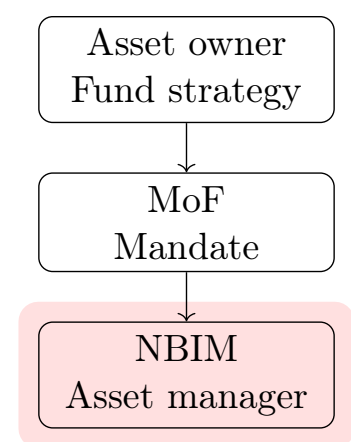

Figure 1: Focus area of the active-management review

Previously, three similar expert groups have worked with similar mandates (see Ang, Goetzmann and Schaefer, 2009; Ang, Brandt and Denison, 2014; Dahlquist and Ødegaard, 2018). ${ }^{1}$ In our opinion, the previous reports and the reporting from NBIM give comprehensive descriptions of NBIM's active management and use state-of-the-art methodologies from the academic literature to evaluate NBIM's performance. We replicate, update, and extend the previous analyses. Instead of organizing the different parts of the report around different evaluation measures (e.g., factor analysis), we structure the report around the Fund's portfolio structure. For example, when examining the equity portfolio, we measure the equity-portfolio performance using several evaluation techniques. Furthermore, in our report, we dig deeper into the Fund's substrategies (e.g., equity-enhanced indexing) and try to identify how important these strategies and substrategies are for the overall outcome. Finally, we evaluate NBIM's active management based on a sample period up to and including September 2021.

\footnotetext{
${ }^{1}$ NBIM also performs self-assessments, see NBIM (2017) and NBIM (2021d) for the last two.
} 
One way of interpreting the Fund's setup is that the MoF has developed a mandate they can delegate to different asset managers. Since the Fund's inception in 1998, NBIM has been the only asset manager asked to execute the mandate.

In our assessment, we compare NBIM's active management with the benchmark portfolio specified in the mandate. NBIM's mandate provides neither an active return target nor an information ratio $(I R)$ target. We as a review committee cannot, therefore, evaluate the size of the active returns. Lacking anything better, we compare the active returns to a threshold of zero.

We employ several quantitative and qualitative analyses. We use the term value creation (destruction) when the NBIM portfolio is performing better (worse) than the benchmark alternative. We can measure value creation in its monetary value in NOK or USD or in percentage of assets under management (AUM), the return. When considering the monetary value, we use the term value added to measure to what extent the NBIM alternative performs better than the benchmark. When we compare the alternatives in the return space, we identify a positive (negative) active return if value is created (destroyed).

We focus on three main questions in the assessment:

- What is the Fund's realized value creation?

- Why and how is this result achieved?

- How can the Fund's value creation be improved?

Based on the three questions above and our interpretation of the review committee's mandate, we develop the outline illustrated in Figure 2. In Section 3, we start by documenting the Fund's realized value creation and assessing the quality of active management at the overall Fund-portfolio level. Since the value-creation process consists of many different investments, it is important to understand the underlying processes. In Section 4, we examine the managed-equity portfolio and in Section 5 the substrategies within that portfolio. In Section 6, we examine the managed fixed-income portfolio and the corresponding substrategies in Section 7.

\section{Section 3}

\section{Fund portfolio}

Section 4

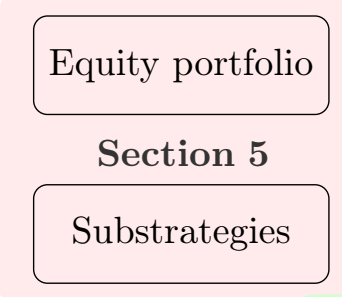

\section{Section 8}

Section 6

Fixed-income portfolio

Section 7

Substrategies

\section{Real-estate portfolio}

Figure 2: Roadmap of the review

Given how the Fund is organized, the real-estate portfolio is one of the active-management strategies. That portfolio's benchmark is a combination of equity and fixed income in line with the opportunitycost model advocated by Ang et al. (2014). Consequently, the active management of the real-estate 
portfolio is related to the equity and fixed-income portfolios. We thoroughly explain and examine the real-estate portfolio in Section 8. Finally, in Section 9, we shift the focus from the past to the future. Here, we list and summarize our key observations and suggestions from the quantitative analysis and the qualitative assessment of NBIM's active management. Section 10 concludes.

\subsection{Performance-evaluation measures}

There is no consensus in the academic literature on a particular method of measuring and reporting portfolio performance (see, e.g., Elton and Gruber, 2020, for a recent review of the relevant literature). Therefore, we emphasize the importance of using alternative approaches, methods, models, and specifications. When documenting the realized value creation, we present gross and net active returns and value added. Active returns are the portfolio returns minus the benchmark returns. We denote the portfolio returns as total returns. ${ }^{2}$ Gross active returns are the active returns after transaction costs but before management costs; see the discussion in Section 2.3. Net active returns are the active returns after management costs. Value added measures how much value the Fund has generated in NOK for a given period (typically a month) by deviating from its benchmark portfolio (see Berk and van Binsbergen, 2015).

When explaining the results, we use various performance measures. The measures mentioned above (returns and value added) can not only be used to document realized results, but also to evaluate performance. These measures are simple and do not rely on any estimated parameters. To avoid mistaken inferences, we use statistical inference to investigate whether the performance measures reliably indicate the skill to add value. To evaluate the active-return performance, we rely on the null hypothesis that the true average active return is zero and the alternative hypothesis that it is not zero. The null hypothesis is that the active return can be ascribed to "luck," while the alternative hypothesis says that the active return can be ascribed to "skill." The $p$-value of the corresponding test statistic provides evidence regarding the null hypothesis. A low $p$-value indicates that we reject the null hypothesis. It is common to use a threshold significance level of .05 as the threshold for a low $p$-value. This threshold means that 1 in 20 conclusions that NBIM has skill are mistaken; it actually has been lucky. The choice of the appropriate significance level is to some extent arbitrary, so we report the $p$-values such that the reader can form his or her own opinion (Wasserstein and Lazar, 2016).

We now turn to risk-adjusted performance measures. The first risk-adjusted performance measure is the information ratio ( $I R$; Grinold, 1989). This measure divides the mean active return by its standard deviation. The standard deviation of the active return is often denoted the tracking error or the active risk. A second risk-adjusted performance measure is Jensen's alpha (Jensen, 1968). Jensen's alpha denotes the average return on the portfolio minus the return predicted by the capital asset pricing model (CAPM). The CAPM return depends on the portfolio's estimated beta and thereby on its market risk. A third risk-adjusted performance measure related to Jensen's alpha is the appraisal ratio $(A R)$. The $A R$ is equal to Jensen's alpha divided by the unsystematic risk of the portfolio (i.e., the standard deviation of the residual from the CAPM regression). ${ }^{3}$ The fourth risk-adjusted

\footnotetext{
${ }^{2}$ The total return is the amount of value the investor earns. Sometimes, the term excess return is used to describe active return. However, we follow the literature and use excess return to describe the return above the risk-free interest rate. ${ }^{3} A R$ and $I R$ are linked. If beta is greater than one and the average excess return is positive, then $A R$ is always lower than IR (see Goodwin, 1998).
} 
performance measure is the Sharpe-ratio, which is targeted at the total return and risk level. The Sharpe ratio divides the mean portfolio excess return by the standard deviation of the return. Thus, the Sharpe ratio measures the excess return per unit of risk and is the most straightforward measure of the trade-off of portfolio return to total volatility. ${ }^{4}$ The difference between the Sharpe ratio for the portfolio and the benchmark is the Sharpe ratio difference.

In our analysis, we provide two regression-based risk-adjusted performance measures: The first is Jensen's alpha mentioned above where we risk-adjust only for the benchmark, similar to the CAPM model. Jensen's alpha is the estimated intercept in the regression of the portfolio excess return on the benchmark excess return. The second measure is a factor model in which we adjust for several additional risk factors. Here, we regress the active returns on a number of risk factors. We interpret the estimated slope coefficients as the active exposures to the systematic factors over the sample period and the estimated intercept (denoted alpha) as the performance attributable to the Fund's value creation over and above the exposure to the chosen set of risk factors. We base our factor analysis on the recommendations in Dahlquist et al. (2015). NBIM also bases its performance evaluations on the same factor models in their reports (see NBIM, 2020a, 2021d).

We have made various decisions regarding which types of analysis to include in this report. For example, we do not explicitly attribute value creation to selection and timing, but mention when a given strategy is based on either. The structure of the Fund's investments and the findings of Dahlquist and Ødegaard (2018, Section 8) suggest that not much value creation occurs from timing between countries or sectors. ${ }^{5}$ Therefore, we do not consider market timing. We fully focus on time-series analysis of portfolio returns. ${ }^{6}$

\section{$1.3 \quad$ Literature}

In this section, we briefly present relevant theoretical and empirical literature regarding active management. Unlike passive asset managers, active asset managers use and interpret information to try to predict future returns. Passive managers hold the market or benchmark portfolio. Active managers earn their title by allocating more to assets that they feel most strongly about.

\section{Theory}

Financial markets are efficient if all available information is embedded in the prices (see Fama, 1970). Due to competition in financial markets, it is reasonable to believe that systematic mispricing does not exist over long periods of time. Financial markets, in that sense, are relatively well functioning.

In an efficient market, investors' expectations of profits and uncertainty are reflected in market prices. These prices also change in line with new information and new insights. Investors have different perceptions about the future and, therefore, about the correct price. These expectations do not have to be rational and can both under- and overestimate a company's future opportunities. A transaction

\footnotetext{
${ }^{4}$ We use the US one-month T-bill rate as a proxy for the risk-free interest rate. The one-month T-bill rate is available from Kenneth French's data library.

${ }^{5}$ Brinson, Hood and Beebower (1995) and others have examined the question of selection versus timing in multiple asset classes. However, for the Fund, this rebalancing decision is taken by the MoF, and is therefore not part of the Fund's active management decision. In Section 9, we briefly comment on the timing between substrategies.

${ }^{6}$ Even if holdings-based methods can help calculate the value of active managers' decisions, this analysis also has important imperfections (see Cremers, Fulkerson and Riley, 2019).
} 
takes place at a price where an investor who has a positive opinion about the future will buy and an investor who has a negative view will sell. However, although investors may have different expectations, the competition in financial markets drives prices to their "fair" level.

With well-functioning markets, investors with market exposure are compensated for the associated risk and have no reason to expect a better trade-off between return and risk by choosing a portfolio other than the market portfolio. In this efficient-market context, is it not possible for a manager to consistently achieve active returns. Systematically delivering a higher return than the broad market would require that the manager has access to information that is not publicly available and is not yet reflected in market prices.

If markets were fully efficient, all investment strategies would generate zero alphas before expenses. An asset manager who incurs costs for active management would then earn a negative net alpha. Therefore, all rational models of active asset management assume a certain degree of market inefficiency. This inefficiency is the rationale for the existence of the active-management and hedge-fund industries. Somebody must acquire and process information and to create a market equilibrium (see Grossman and Stiglitz, 1980). In Grossman and Stiglitz's (1980) model, prices do not appear to be fully efficient but, instead, appear to exhibit an "equilibrium degree of disequilibrium." Gârleanu and Pedersen (2018) suggest that large institutional investors can capture economic rents by transforming all relevant information into prices. This potentially creates a space for active management.

\section{Empirical literature}

Many institutional asset managers invest actively. ${ }^{7}$ Active investments go against the financial theory that suggests that there is only limited scope for active management. Sharpe's (1991) "arithmetic of active management" presents an adding-up constraint that implies that active and passive investors must earn identical gross returns. French (2008) uses related reasoning to point out that active management before expenses is a zero-sum game and is a negative-sum game after expenses.

The literature suggests two reasons why this adding-up constraint does not hold strictly. First, there are costs associated with passive management. Pedersen (2018) and Berk and van Binsbergen (2015) argue that the market itself changes over time as new firms are added, old firms are delisted, new shares are issued, and old shares are repurchased. Hence, passive investors also need to trade to track the market. In turn, passive investors may lose to active investors if they trade at systematically less favorable prices than those obtained by active investors.

Second, some institutional investors might win at the expense of others, often named noise traders (see Black, 1986). The fraction of individual investors in the market can be a proxy for noise traders. Barber and Odean (2013) find that these investors lose because they suffer from several behavioral biases. Gerakos, Linnainmaa and Morse (2021) suggest that institutional investors earn positive alphas at the expense of less sophisticated investors (see also Fama and French, 2010; Akepanidtaworn, Mascio, Imas and Schmidt, 2021). However, there is mixed evidence regarding the result of institutional investors' active management. Dyck, Lins and Pomorski (2013) find that pension funds earn positive alphas on the assets that they delegate to active strategies in EAFE (European, Australasia, and the Far

\footnotetext{
${ }^{7}$ Section I in Ang et al. (2009) gives an overview of the active-asset-management literature. Cremers et al. (2019), Antón. Cohen and Polk (2021), Berk, Van Binsbergen and Miller (2020), and Ang, Chen, Gates and Henderson (2021) review the mutual-fund literature.
} 
East) and emerging markets. They would not have done better managing passive strategies in-house. Others find opposite results (Goyal and Wahal, 2008; Busse, Goyal and Wahal, 2010; Lewellen, 2011; Andonov, Bauer and Cremers, 2012; Jenkinson, Jones and Martinez, 2016).

\section{The Fund's potential advantages}

There are various forms of active management. Inspired by the decomposition of Ang et al. (2014, Eq. (2)), we decompose active management into four subsets, 1) extended index management, 2) static factor betting, 3) timing of factors, sectors, and countries, and 4) security selection. An important question is whether the Fund has advantages that can be exploited in these active strategies?

Extended index management (or enhanced indexing) is a common strategy also executed by ordinary index-fund (passive) managers. In the equity and fixed-income portfolio, NBIM use similar strategies. Enhanced indexing strategies aim to mimic the benchmark in a "smart" way without materially adding tracking error (see Pedersen, 2018, for an overview of enhanced indexing activities). Chen, Noronha and Singal (2006) show wealth transfers from passive funds to active funds due to arbitrage activity around the reconstitution of the S\&P 500 index and the Russell 2000 index. Elton, Gruber and Souza (2021) summarize the performance of enhanced index funds. Our interpretation is that the Fund has ample resources because of its size and reputation and can afford patience in their investment process, which can lead to advantages related to the enhanced indexing strategies.

Two of the previous reports have recommended factor investing. NBIM has experimented with factor investing. Even though this is a relatively new area of active management (see, e.g., Ang, 2014), it is our understanding that over the last few years, several academics have become skeptical of factor investing (see, e.g., Arnott, Harvey, Kalesnik and Linnainmaa, 2019). Nonetheless, some recent papers (e.g., Baltussen, Swinkels and Van Vliet, 2021) show long-horizon payoffs of these factor strategies, despite the breakdown of some factors in the last decades. Our interpretation is that factor strategies, as well as timing strategies, are a very competitive area in which NBIM does not necessarily have any clear comparative advantages. It is also unclear, going forward, exactly which factors yield positive risk premiums and whether these premiums are time varying.

For security selection, the portfolio manager considers investment opportunities from a bottom-up perspective by finding attractive securities and then aggregating these to the portfolio level. The academic literature related to security selection discusses many systematic yet fundamentals-based strategies. In the early part of this literature, Treynor and Black (1973) picked up this thread from Markowitz (1952) and applied a level of mathematical rigor to security selection. Today, considerable literature from the practitioner's side proposes various methods for designing security-selection portfolios.

For equity mutual funds, Daniel, Grinblatt, Titman and Wermers (1997) and Wermers (2000) find that, on average, funds select stocks that outperform the market. However, Fulkerson (2013) shows the overperformance resulting from this skill has declined moderately through time. He also finds that the majority of stock selection skill tends to come from selecting stocks within industries. Dellva, DeMaskey and Smith (2001) find evidence of stock-picking ability in sector funds, consistent with manager expertise in the sector. Antón et al. (2021) find that stock-picking skills are especially pronounced in the largest positions in a fund's portfolio. However, with concentrated portfolios, one 
can miss the few outperforming stocks (see Bessembinder, 2018, 2021). Kacperczyk, Nieuwerburgh and Veldkamp (2014) find that outperforming managers apply a set of time-varying skills. Stock picking is evident in expansions, and market timing is evident in recessions. By examining fixed-income fund buying and selling, Moneta (2015) finds that fixed-income funds generate positive alphas before costs. After cost, the results are more ambiguous (see, e.g., Chen, Ferson and Peters, 2010).

The characteristics of the Fund, such as its long investment horizon, the patience associated with the governance structure, and its size-related resources can positively influence active returns from security selection. These characteristics support NBIM in exploiting the two primary sources of repeatable trading profits suggested by Pedersen (2015): compensation for liquidity risk and information advantage. For example, Cremers and Pareek (2016) show that active longer-term managers have better results than shorter-term managers. However, there may also be diseconomies of scale. Since transaction costs increase with size, most trading security selection strategies have a limited capacity (see Pástor, Stambaugh and Taylor, 2015). Moreover, security selection is a highly competitive exercise. ${ }^{8}$

\footnotetext{
${ }_{8}^{8}$ Except for the potential benefits of making money, there are potentially also positive externalities from pursuing active management (see Section II in Wermers, 2021). We return to this topic in Section 9.
} 


\section{Overview of the Fund}

The Fund was founded in 1986 by the Norwegian government to accumulate surplus from the Norwegian oil revenues. The Norwegian government decided that this windfall gain should benefit future generations through the Fund. In 1996, the first capital injection took place. As shown in Figure 3, the Fund's market value (assets under management, AUM) has grown steadily since then, due to both new capital injections and (predominantly) to high financial returns on the Fund's investments. ${ }^{9}$

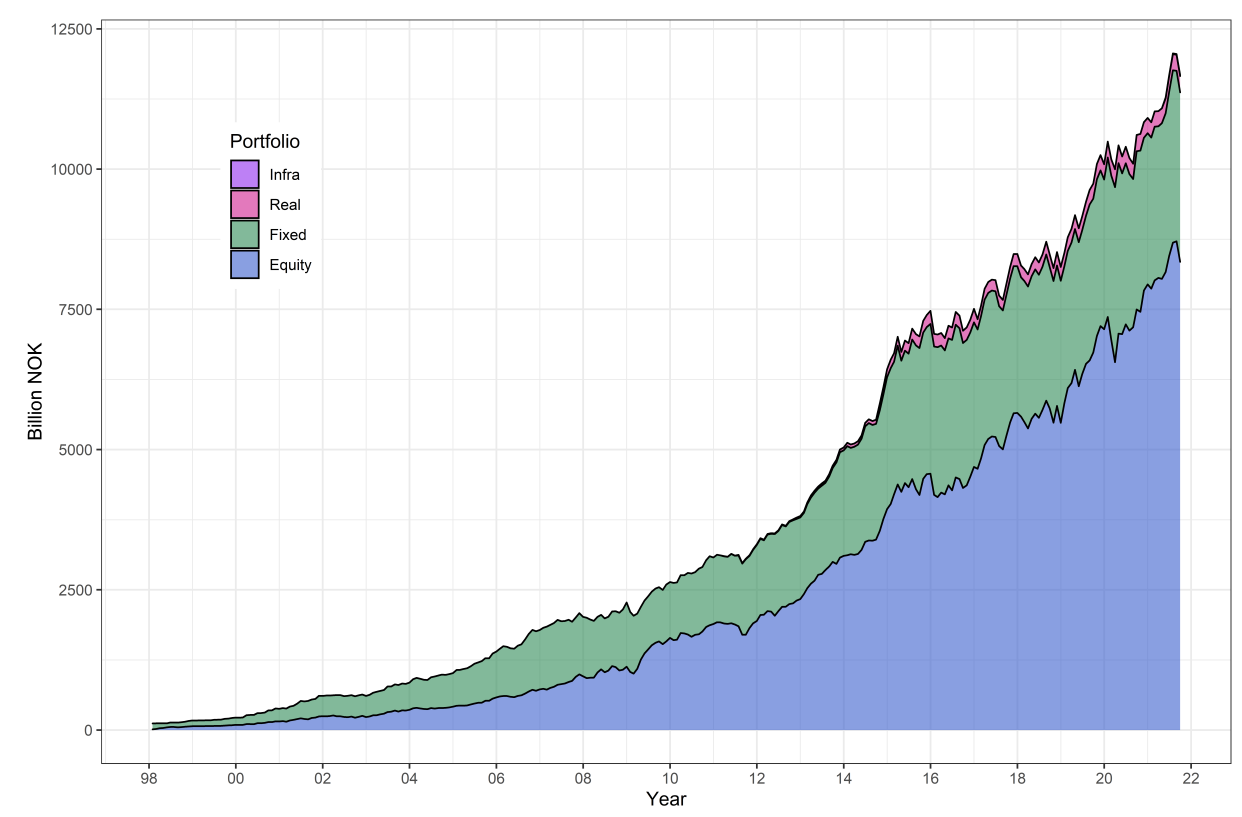

Figure 3: Fund AUM across asset classes

The Fund's initial investments were allocated to government fixed-income securities. As the Fund grew, allocations expanded to other asset classes. In January 1998, the Fund began to acquire equity, starting with a strategic asset allocation of $40 \%$ equity and $60 \%$ fixed income. This starting point (1998) is also the starting date for our analyses. As shown in Figure 25 in Appendix C, the allocation to equity has continued to grow. Today, the strategic allocation to equity is $70 \%$. In 2010 , the MoF authorized the addition of unlisted real estate to the portfolio, and, finally, during the summer of 2021, the first renewable infrastructure investment took place. ${ }^{10}$

The mandate does not mention an active return target nor an $I R$ target, only an ex-ante trackingerror limit of $1.25 \%$ (see Ministry of Finance, 2021, Section 2.4, paragraph 6). The tracking-error limit has been quite stable over time. It changed at some point from $1.50 \%$ to $1.00 \%$, and is now at $1.25 \%$. Since the ex-ante tracking-error measure has its weaknesses, more risk measures have been added. For example, the MoF mandate demands that NBIM establish methods for calculating extreme-event risk analysis (Section 3.3 in the mandate). ${ }^{11}$

\footnotetext{
${ }^{9}$ See the 2020 annual report for the different sources of the AUM (NBIM, 2020b, Chart 3).

${ }^{10}$ In connection with the Fund's 25th anniversary, the Fund has published its own history in several review papers (see NBIM, 2020g,c,f,e,d,h,i, 2021c).

${ }^{11}$ We have received data from NBIM with their ex-ante tracking errors and extreme-event risk measures without explicitly using them in the assessment. For tracking errors, we calculate our own ex-post tracking errors. NBIM discusses extreme-risk measures in their self-assessment (NBIM, 2021d).
} 


\section{$2.1 \quad$ Data}

Most of the data we use come from NBIM. Some data series are publicly available on NBIM's home page, but we have also requested and been provided access to nonpublic data. For more details about the data, see Appendix B.

At the Fund, equity, and fixed-income levels, we use monthly returns and AUM data from January 1998 through September 2021, providing us with 285 observations. In most of the analysis, we also examine the 57 observations from the most recent period, January 2017-September 2021. We explicitly note analyses based on data available only for shorter periods.

The Fund is organized such that its portfolio can be decomposed into many subportfolios. At the highest level, we have the Fund portfolio with a MoF-provided benchmark. Then we have the equity and fixed-income portfolios, which NBIM denotes as the asset-class portfolios. Next, are the so-called managed portfolios for equity and fixed income. Due to the organization of the real-estate and infrastructure portfolios, they are different from the equity and fixed-income portfolios. We return to this difference later.

Beneath the equity and fixed-income aggregation level are the subportfolios for which we report results. For these subportfolios, the sample period is shorter and begins in January 2013, yielding 105 observations. We have also seen examples of internal reports on portfolio performance all the way down to individual portfolio managers. We perform no analyses at this lower aggregation level in this report.

The Fund provides its returns in USD, NOK, and in the Fund's so-called currency basket. We have chosen to present our return results in USD and therefore use USD returns for our calculations. Our primary analysis is on active returns, and the correlations between active returns in USD, NOK, and the currency basket are, respectively, high. As a robustness check, we repeated most of the analysis using returns measured in the Fund's currency basket (available in the appendixes). We find only minor differences. The main reason for going with the USD results is that this makes it easier to compare our results with other studies. ${ }^{12}$ For further descriptions of the currency conversion, see Appendix B.

For the analysis in monetary terms, we report the value added in NOK. That is, we multiply the active returns (in USD) by the AUM in NOK. A cleaner calculation would multiply the active returns in NOK by the AUM in NOK; however, as argued above, there is a high correlation between active returns in NOK and USD. We use NOK for monetary terms, as it is easier for the Norwegian public to understand. Another potential, but in our opinion unimportant, source of error is our use of monthly rather than daily data. Using the monthly frequency, we may miss, for example, timing elements of the strategies.

\subsection{Benchmark portfolios}

According to the CAPM, the most desirable portfolio in an efficient market is the global market value-weighted portfolio. For various reasons, the benchmark designed by the MoF includes many implicitly "active" decisions, such as the underweighting of the US market relative to the world market. We take the benchmark developed by the MoF for granted and use it at face value. We define the

\footnotetext{
${ }^{12}$ The factor returns on French's homepage are in USD. By using the same currency, we avoid the need to convert to another.
} 
active return at time $t, R_{t}^{\mathrm{a}}$, as the difference between the Fund's total return, $R_{t}$, and the benchmark return, $R_{t}^{\mathrm{b}}$. The MoF benchmark's asset weights drift within a rebalancing band. ${ }^{13}$

The design of appropriate benchmarks has a long history of debate (see, e.g., Sharpe, 1992). We stick to the properties listed in Wermers (2011) and repeated in Appendix C. Some essential properties of a valid benchmark are that the benchmark should be unambiguous, measurable, specified in advance, tradable, and appropriate. At the Fund level, the MoF's benchmark is unambiguous, measurable, specified in advance, and appropriate. It is also tradable, but at some cost. We will return to the costs of passively managing the benchmark in Section 2.3.

In general, shifting from an external benchmark to internally developed benchmarks involves a potential problem of self-selected benchmarks. Sensoy (2009) shows that asset managers of mutual funds frequently supply erroneous benchmarks. For any fund with an inaccurate match, the prospectus benchmark could over- or underestimate the true performance. We have no reason to believe that NBIM deliberately chooses "favorable" internal benchmarks. Based on our understanding, a complex process between an investment committee and the managers for the strategies decides the internal benchmarks. Moreover, all the internal benchmarks add up to the MoF's mandated benchmark. ${ }^{14}$ In our analysis, we also use the internal benchmarks at face value. However, to reduce the potential for inappropriate self-selected benchmarks, and not least the suspicion of such, we suggest a more accessible and more transparent process for internal benchmarks in Section 9.

Another issue that complicates the choice of internal benchmarks is the real-estate and infrastructure funding models. The Fund's equity and fixed-income portfolios fund these real assets. The Fund uses the same benchmark as its funding for the real assets. Thus, a difference exists between the benchmarks for equity and fixed-income based on the MoF benchmark and the internal benchmarks. We return to the benchmarks in Sections 4 for equity, 6 for fixed income, and 8 for real estate. We also come back to the funding model in Sections 8 and 9.

\subsection{Costs}

We split the discussion of costs into two parts, the Fund's operating cost and the cost of the counterfactual (benchmark) portfolio.

\section{Operating costs}

The Fund's operating costs can be divided into two cost categories: the transaction costs involved in trading financial assets and the costs of running the Fund's operations, the management costs. The Fund's returns are net of transaction costs, while the management costs are published separately. Below, we use "costs" as shorthand for management costs. NBIM (2020c) breaks transaction costs into three components; commissions, taxes, and implementation shortfalls. The latter reflects the difference between the price at the time of the order and the price achieved in the market. ${ }^{15}$ Management costs are all costs related to running the Fund and also include the performance-related costs. Thus, an increase in costs can be caused by increased value creation from active management. The cost data

\footnotetext{
${ }^{13}$ The MoF's mandate decides these rebalancing bands. The benchmark with fixed weights is the strategic benchmark. For a helpful description of splitting active returns into different parts, see the figures in Section I of Ang et al. (2014).

${ }^{14}$ The benchmark design for the active investment process is described in NBIM (2014).

${ }^{15}$ See Chart 75, 77, or 78 in NBIM (2020c) for the development of the size of the transaction costs.
} 


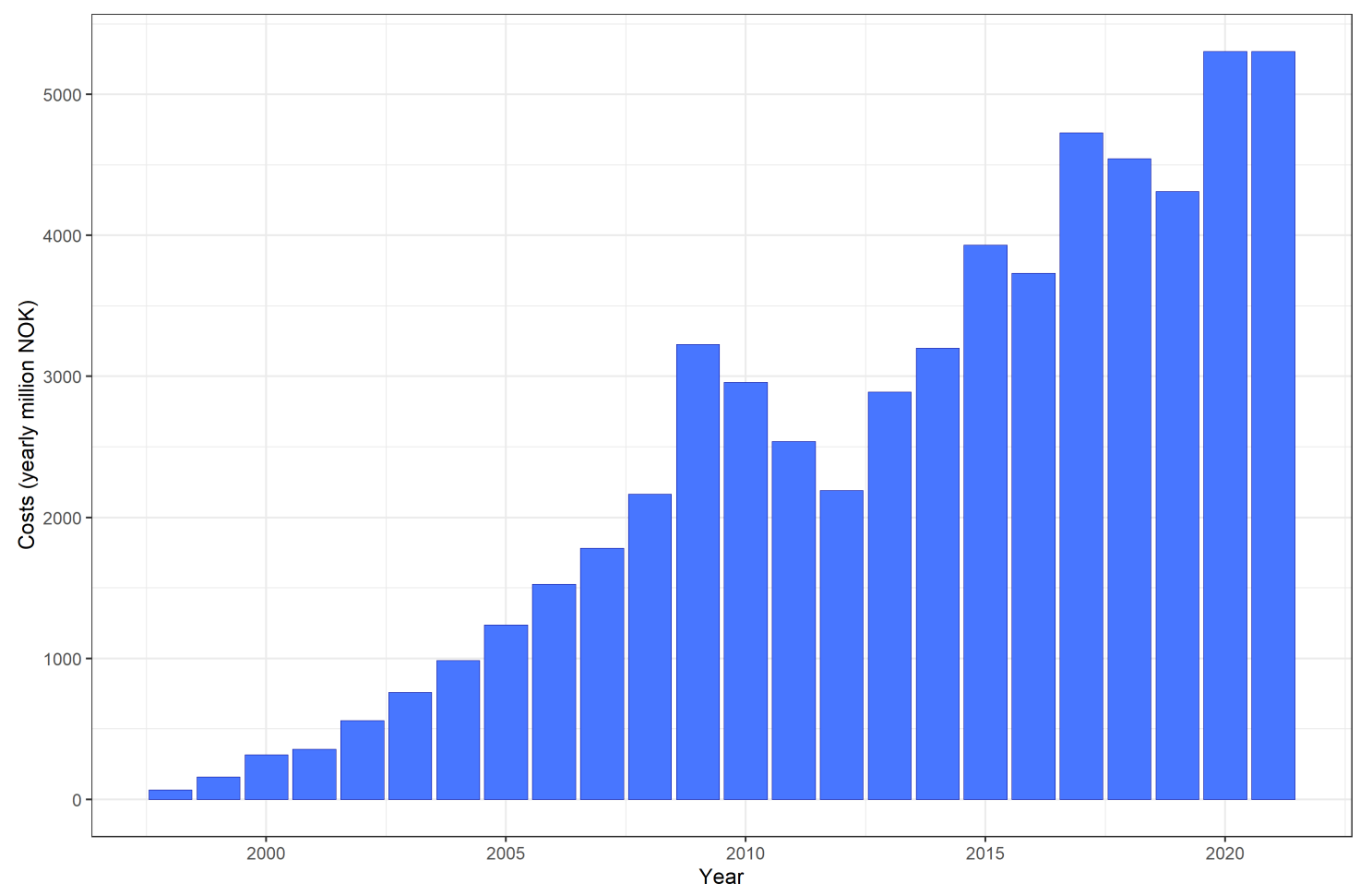

Figure 4: Fund annual management costs in NOK

are published by NBIM annually. For the monthly analysis, we divide the costs evenly over 12 months. Since we do not yet have access to the costs for 2021, we use the cost data for 2020. In Figure 4, we see that the Fund's annual costs in NOK have increased along with the AUM.

The Fund also allocates the costs to its different portfolios. We take the cost allocations for granted, although the allocation process can always be discussed. In Figure 5, we illustrate the development of the costs in percentage of AUM where the Fund's costs are earmarked to the various asset classes. Costs at the total level are very stable around $0.05 \%$ in the past 10 years. Real estate shows the

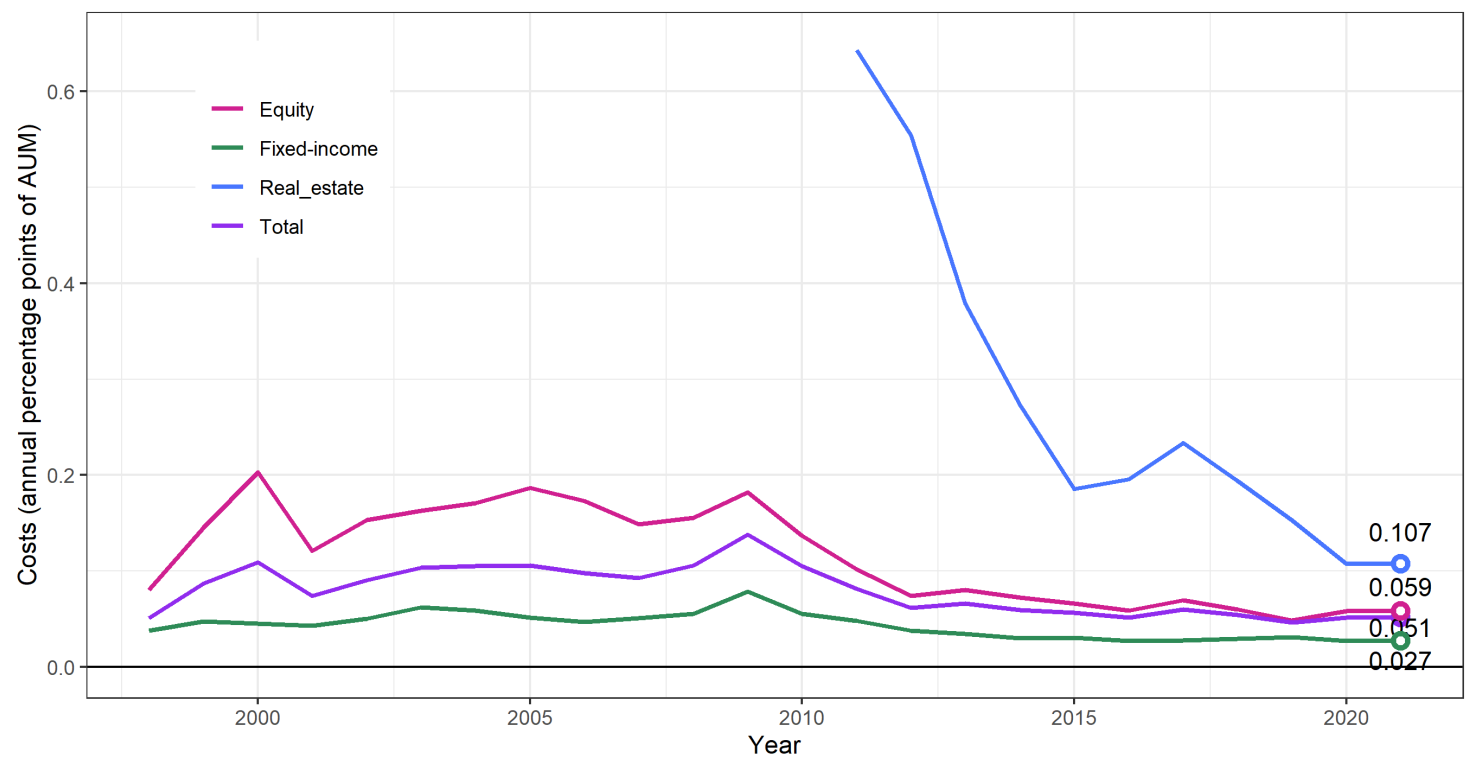

Figure 5: Fund annual management costs as a percentage of AUM 
highest cost level (but with a sharp downward trend), whereas fixed income has the lowest cost level $(0.03 \%)$. It is particularly interesting to examine the value creation before and after costs for the internal and external-security-selection strategies, because the Fund has allocated most of its costs (relative to AUM) to these strategies.

\section{Benchmark costs}

As we are comparing the Fund to its benchmark, it is important to make a fair comparison regarding costs. While the Fund's returns account for transaction costs, the benchmark portfolio returns do not reflect these costs. The benchmark portfolio also has costs related to investing in companies and costs associated with changes in the portfolio weights. The true value creation is thus the active return net of all its costs measured against an estimate of how much it would cost to implement the benchmark.

As benchmark returns are what a fictitious portfolio would return, it is not straightforward to estimate the costs of passively implementing the portfolio. NBIM (2020c, p. 140, Chart 141) reports the estimated cost for managing a passive benchmark, identifying two main types of expenses: transaction costs related to inflows and extraordinary benchmark changes and transaction costs associated with replication of the benchmark. The estimated transaction costs for the period 1998-2019 are 0.08\% of AUM. However, Chart 142 shows decreasing transaction costs over time down to $0.02 \%$ in $2019 .{ }^{16}$

Since we include income from security lending in the Fund's returns, we should also add a similar income stream to the benchmark; a pure index fund can also lend out its securities. Later in the report, we estimate the security-lending returns for the period from 2013. Similarly, these activities are described in NBIM (2020c, p. 157): From Chart 186 it seems the Fund gets a higher return from lending out equity than the market average. Thus, even if the Fund were run like a pure index fund, we estimate that the Fund should expect to earn between $0.04 \%$ and $0.06 \%$ from lending out assets.

Therefore, we suggest that transaction and management costs associated with holding the benchmark and the income from security lending are of about the same size, and thereby cancel out. For this reason, we do not adjust the benchmark for costs. ${ }^{17}$ To be clear, the gross active return analysis we present compares the Fund's actual portfolio returns after transaction costs, but with security lending, with the unadjusted benchmark returns. When we report net active returns, we use the Fund's returns after transaction and management costs, but with income from security lending.

\subsection{Realized total return and risk}

Before examining the value creation, we examine the Fund's total gross and net returns and the benchmark's total returns. In Table 1, we present summary statistics of annualized monthly Fund, equity, and fixed-income returns. Throughout this report, we annualize returns by multiplying monthly returns by 12 and monthly standard deviations by the square root of 12 . This may be questioned with times series that are not independent and identically distributed (IID) (see Lo, 2002). We will come back to the IID assumption, for example, when measuring the autocorrelation of active returns.

\footnotetext{
${ }^{16}$ Also, CEM (2019) reports a cost estimate for the benchmark.

${ }^{17}$ The estimated costs of managing the actual benchmark could, in principle, be added to the performance of the Fund (see Appendix A in Fama and French, 2010, for a discussion of costs in mutual funds and efficiently managed passive benchmarks). We have decided not to do this.
} 


\begin{tabular}{lccc|rcc|ccc}
\hline & \multicolumn{3}{c|}{ Fund portfolio } & \multicolumn{3}{c|}{ Equity portfolio } & \multicolumn{2}{c}{ Fixed-income portfolio } \\
\cline { 2 - 9 } Return & Gross & Net & Bench & \multicolumn{7}{c}{ Gross } & Net & Bench & Gross & Net & Bench \\
\hline Gull sample (1998-Sep 2021) \\
\hline Arit. mean & 6.53 & 6.45 & 6.28 & 7.15 & 7.04 & 6.76 & 4.69 & 4.65 & 4.54 \\
Std. dev. & 10.11 & 7.03 & 6.83 & 8.55 & 8.44 & 8.11 & 4.93 & 4.88 & 4.75 \\
Sharpe ratio & 0.50 & 0.49 & 0.48 & 0.41 & 0.40 & 0.39 & 0.46 & 0.46 & 0.45 \\
$N$ & 285 & 285 & 285 & 284 & 284 & 284 & 285 & 285 & 285 \\
& 10.10 & 10.05 & 9.84 & 12.46 & 12.40 & 12.24 & 4.23 & 4.20 & 3.96 \\
Geo. mean & 10.45 & 16.61 & 16.61 & 16.33 & 6.83 & 6.83 & 6.60 \\
Arit. mean & 10.73 & 10.68 & 10.46 & 13.64 & 13.58 & 13.39 & 4.33 & 4.30 & 4.07 \\
Std. dev. & 11.32 & 11.32 & 11.22 & 15.39 & 15.39 & 15.21 & 4.59 & 4.59 & 4.56 \\
Sharpe ratio & 0.85 & 0.85 & 0.84 & 0.82 & 0.81 & 0.81 & 0.71 & 0.70 & 0.65 \\
$N$ & 57 & 57 & 57 & 57 & 57 & 57 & 57 & 57 & 57 \\
\hline
\end{tabular}

Notes: The table shows statistics for the gross, net, and benchmark returns for the total Fund portfolio, the equity portfolio, and the fixed-income portfolio. The table shows the annualized geometric mean, arithmetic mean, standard deviation, Sharpe ratio, and number of observations, $N$. All returns are expressed in USD percentages.

Table 1: Total return and risk

For the entire period, we find that the annualized arithmetic mean returns of the Fund's total gross, net, and benchmark portfolios are $7.11 \%, 7.03 \%$ and $6.83 \%$, respectively. By construction, the geometric mean returns are smaller than the arithmetic means. In the remaining analysis, we use arithmetic means (similar to, for example, Dahlquist and Ødegaard, 2018). The annualized standard deviation of the Fund (gross and net) and benchmark portfolio are 10.76\% and 10.45\%, respectively. The higher return on the portfolio has come at the expense of higher risk compared to the benchmark. The portfolio Sharpe ratios are marginally above the benchmark. For the recent sample period, the return and risk are higher for the Fund than the benchmark. Again, the Sharpe ratios are very similar, but at a much higher level, around 0.85. This reflects that the recent sample period has had returns above the long-term average. ${ }^{18}$

For the equity portfolio for the full sample period, the average gross, net, and benchmark returns are $8.55 \%, 8.44 \%$, and $8.11 \%$, respectively. The standard deviations are $16.61 \%$ and $16.33 \%$. Again, the Sharpe ratios are very similar at about 0.40 . For the most recent sample, the returns are very high and the Sharpe ratios are also higher and similar.

For the fixed-income portfolios for the full sample period, the average gross return, net return, and benchmark returns are $4.93 \%, 4.88 \%$ and $4.75 \%$, respectively. The standard deviations are $6.83 \%$ and $6.60 \%$. The Sharpe ratios are almost identical for the fixed-income portfolio and its benchmark, around 0.46 . The Sharpe ratios are higher for the fixed income than the equity portfolio due to the relatively lower standard deviations.

A simple but intuitive framework for an investor not taking other assets (such as human capital and pension liabilities) into account is the expected return-standard deviation setting. The relevant inputs

\footnotetext{
${ }^{18}$ Table 39 in Appendix C reports summary statistics for the Fund and its benchmark measured in the Fund's currency basket instead of in USD. While the mean returns are similar, there are substantial differences in standard deviations. These differences also influence the Sharpe ratios. Still, since we use active return for the performance analysis, none of the currency differences at total return levels impact the results regarding the active returns. In their self-assessment, NBIM report returns in the Fund's currency basket (NBIM, 2021d). We assume that they annualize monthly returns by compounding. For example, the gross total return of $6.31 \%$ and the benchmark return of $6.06 \%$ from our Table 39 then becomes $6.49 \%$ and $6.23 \%$, respectively (similar to Table 4 in Enclosure 1 in NBIM, 2021d). The difference between these two numbers is the active return of $0.26 \%$ (from the same table).
} 
are the investor's risk preferences and expected total return and risk. In this setting, the investor can use the risk-free asset to leverage up and down the tangency portfolio according to his or her preferences. However, it is troublesome for a long-term investor, such as NBIM, to identify a genuinely long-term risk-free asset. Figure 26 in Appendix C illustrates the portfolios (after costs) and the benchmarks in a mean-standard deviation graph with no risk-free asset available. That is, the investor chooses the optimal risky portfolio directly on the efficient frontier.

The overall Fund portfolio's efficient frontier combines the equity and fixed-income benchmark portfolios. The correlation coefficient between the equity benchmark and the fixed-income benchmark is 0.37 for this period. Even if the risk-free alternative is not suitable in this setting, we still report Sharpe ratios using the US one-month T-bill rate as a proxy for the risk-free interest rate in Table 1. We see that the Sharpe ratio differences are negligible. 


\section{Fund portfolio value creation}

In this section, we examine the overall Fund portfolio's active value creation. In subsequent sections, we investigate the Fund's constituents. We use the same methods in our analysis of all the different portfolios, so we only explain them in detail the first time they are used, in this section.

\subsection{Realized value creation}

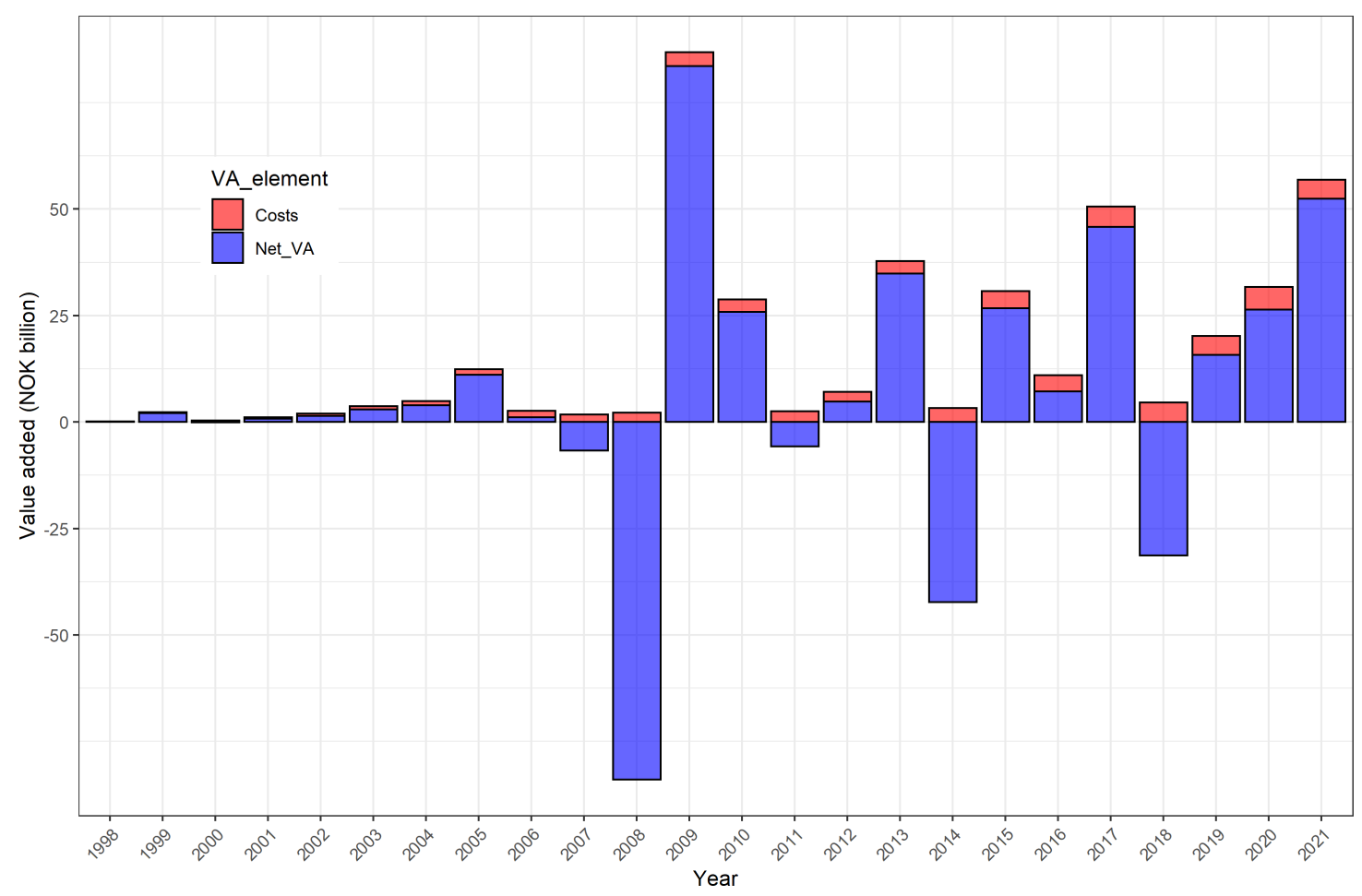

Figure 6: Fund annual value added before and after management costs

In Figure 6 we plot the annual value added from active management. Value added describes how much value the Fund extracts from the financial markets. The monthly value added for period $t+1$ is the product of the active return at month $t+1$ and the Fund's AUM at time $t: V_{t+1}=A U M_{t} \cdot R_{t+1}^{\mathrm{a}}$, based on monthly AUM in NOK and active returns in USD. The annual value added is the sum of the monthly value-added amounts. The blue bars are the net value added, while the red bars are the costs. The sum of the two are gross value added. Until 2007 there were relatively small changes in value added, while in the more recent years, there have been larger variations. This is not strange because the active returns are value weighted with the size of the Fund, which has been increasing. The accumulated gross value added for the period is NOK 228 billion. ${ }^{19}$ The accumulated costs are NOK 58 billion, and thereby the accumulated net value added is NOK 170 billion. For the latest period, i.e., from 2017, the accumulated gross value added is NOK 131 billion, the costs are NOK 23 billion and net value added is NOK 108 billion. The Fund's gross value added has been positive most years except for five years, including the very large negative value added in 2008, and two large negative value added amounts in 2014 and 2018.

\footnotetext{
${ }^{19}$ The accumulated gross value added, net value added, and costs are not directly observed from Figure 6 but are obtained by accumulating the values from each month over the entire sample period.
} 
When we measure performance before and after costs, we answer two different questions. When we want to examine NBIM's investment skill, then the before-cost measures are the more appropriate, while if we are examining whether the Fund has made money, then the after-cost measures are appropriate. Following the arguments of Berk and van Binsbergen (2015), before-cost measures can be viewed as how much the Fund extracts from the capital markets. Whether the Fund has high or low costs does not influence NBIM's skill, but it does influence how much money is left for future consumption by the Norwegian people. Berk and van Binsbergen (2015) further argue that the value-added measure is a better skill measure than the gross active return for very large portfolios because generating a high return is more difficult with more AUM. For example, all else equal, it has been more challenging for the Fund to create a positive active return in recent years when the Fund has been larger than previously when the Fund was smaller.

We show another preliminary illustration of the Fund's value creation in Figure 7, namely the accumulated active returns. Suppose we had invested NOK 1,000 in the Fund's active portfolio at its inception, then this investment would have yielded NOK 1,069 before and NOK 1,049 after costs by September 2021. This represents the additional return we would have earned by investing in the Fund itself instead of in the Fund's benchmark. There is a large decline during the financial crisis in 2008. In contrast, the large downturn in the Fund's total returns during the COVID-19 crisis in March 2020 hardly influences its active returns.

In Table 2 we present the average monthly active return (annualized) and the average monthly value added before and after costs, for the full sample and for the recent sample. We find that the annualized mean active return is $0.28 \%$ before costs, and $0.20 \%$ after costs. The monthly mean value added is NOK 0.80 billion before costs and NOK 0.60 billion after costs. For the recent sample period, the annualized mean active return is $0.27 \%$ before costs and $0.22 \%$ after costs. The average gross

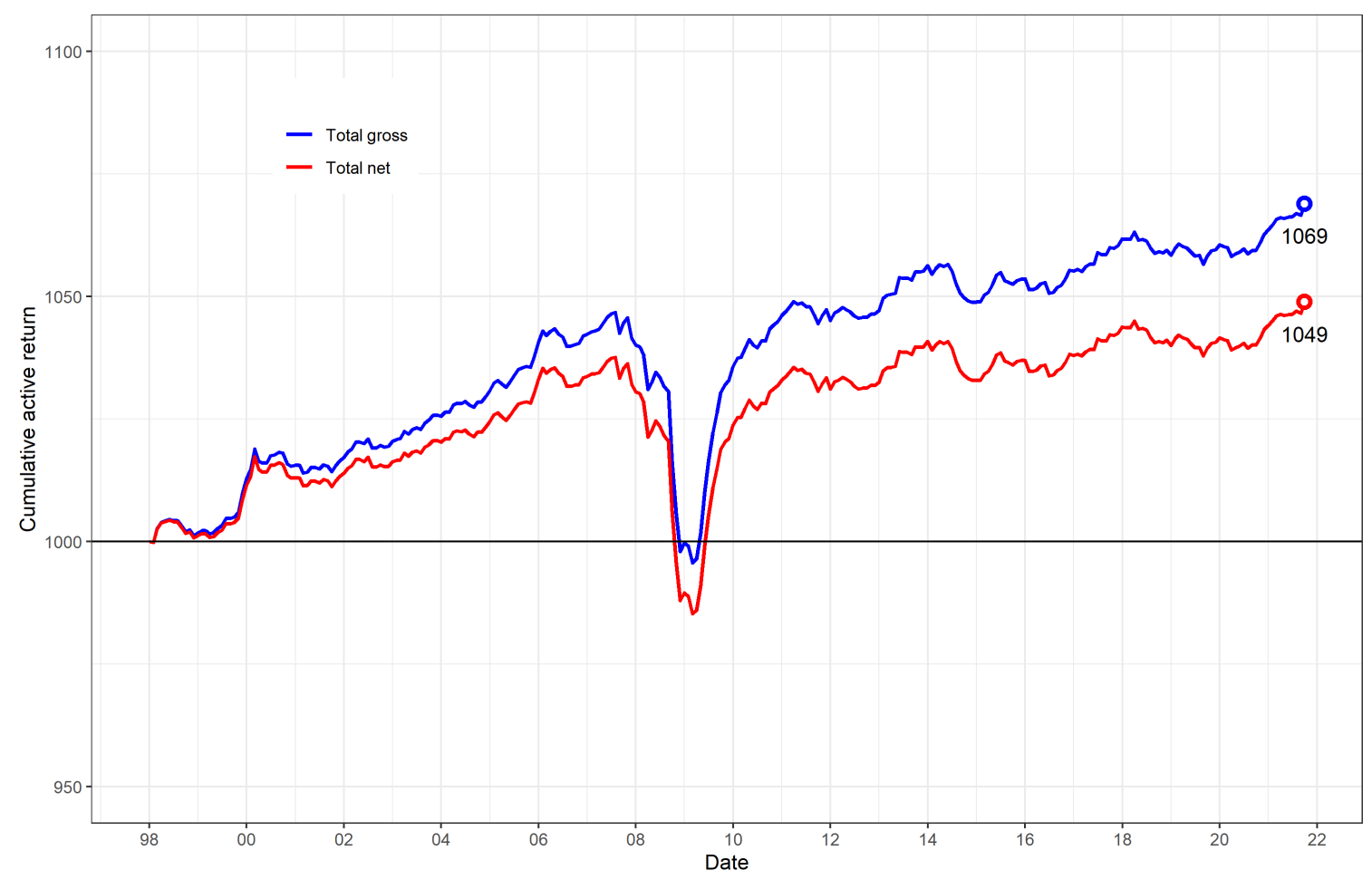

Figure 7: Fund total cumulative gross and net active returns 


\begin{tabular}{lcc|cc}
\hline & \multicolumn{2}{c|}{ Full sample (1998-Sep 2021) } & \multicolumn{2}{c}{ Latest sample (2017-Sep 2021) } \\
\cline { 2 - 5 } & Return & VA & Return & VA \\
\hline Gross & 0.28 & 0.80 & 0.27 & 2.30 \\
Costs & 0.08 & 0.20 & 0.05 & 0.41 \\
Net & 0.20 & 0.60 & 0.22 & 1.89 \\
\hline
\end{tabular}

Notes: The table shows the costs and the gross and net mean annualized active returns (USD) and value added (NOK). Returns expressed in percent, value added in NOK billion.

Table 2: Fund realized mean active returns

value added is NOK 2.30 billion per month before costs and NOK 1.89 billion after costs. The main reason for the greater monthly value added during the latest period is the higher AUM. In Table 40 in Appendix D, we report the same figures in the Fund's currency basket instead of in USD. We see only small differences.

\subsection{Evaluation of active returns}

The realized value creation is one of many scenarios that could have materialized. How this value was created is also important; for example, it can be the result of wild variation or of a more steady development. The standard deviation of the value creation gives a picture of the risk associated with active management.

Table 3 shows the active returns' descriptive statistics. With an annualized tracking error of $0.64 \%$, the annual gross $I R$ is 0.44 , and the net $I R$ is 0.32 for the entire sample period. For the most recent period, the annualized tracking error is 0.32 , and the gross $I R$ is 0.87 and net $I R 0.70$. This period's active return is about the same as its long-run average, but a lower tracking error makes the $I R$ larger than for the entire sample. While the autocorrelation, $A C(1)$, is relatively high for the entire period, it is nearly zero for the most recent period. We will come back to how this change in autocorrelation might influence the hypothesis tests.

\begin{tabular}{lcc|cc}
\hline & \multicolumn{2}{c|}{ Full sample $($ 1998-Sep 2021) } & \multicolumn{2}{c}{ Latest sample (2017-Sep 2021) } \\
\cline { 2 - 5 } & Gross & Net & Gross & Net \\
\hline Mean & 0.28 & 0.20 & 0.27 & 0.22 \\
Std. dev. & 0.64 & 0.64 & 0.32 & 0.32 \\
IR & 0.44 & 0.32 & 0.87 & 0.70 \\
Hit ratio & 0.65 & 0.61 & 0.63 & 0.60 \\
AC $(1)$ & 0.48 & 0.47 & -0.05 & -0.05 \\
Skewness & -2.13 & -2.17 & -0.10 & -0.10 \\
Exc. kurtosis & 17.80 & 17.97 & -0.19 & -0.19 \\
$N$ & 285 & 285 & 57 & 57 \\
\hline
\end{tabular}

Notes: The table shows descriptive statistics for the gross annualized active returns expressed in percent. IR refers to the mean divided by the standard deviation. Hit ratio is the fraction of months with a positive outcome. $\mathrm{AC}(1)$ refers to the first-order autocorrelation. $N$ is the number of observations in the period.

Table 3: Fund summary statistics of active returns

A motive for active management could be good performance when it matters the most to the owner, i.e., in down markets. Figure 8 shows a scatter plot of the monthly active returns against the 


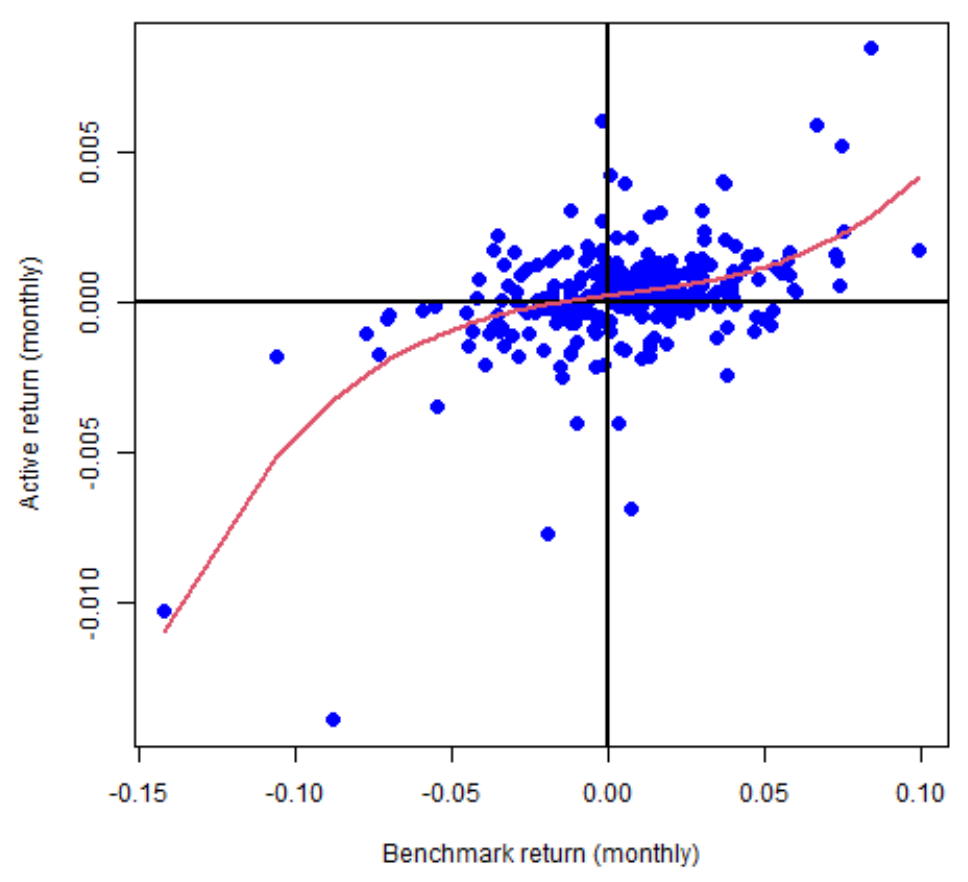

Figure 8: Fund active returns against benchmark returns

benchmark returns. By fitting a third-degree polynomial, we see that the active returns have a weak tendency to be low when the benchmark returns are low, thus, active management does not hedge downside risk.

In Figure 9, the gray bars constitute the histogram of the monthly active returns. The green vertical line goes through zero. We see that the distribution is almost symmetric but with a bit more mass on the positive side. The blue line is the density function of a normal distribution with the same

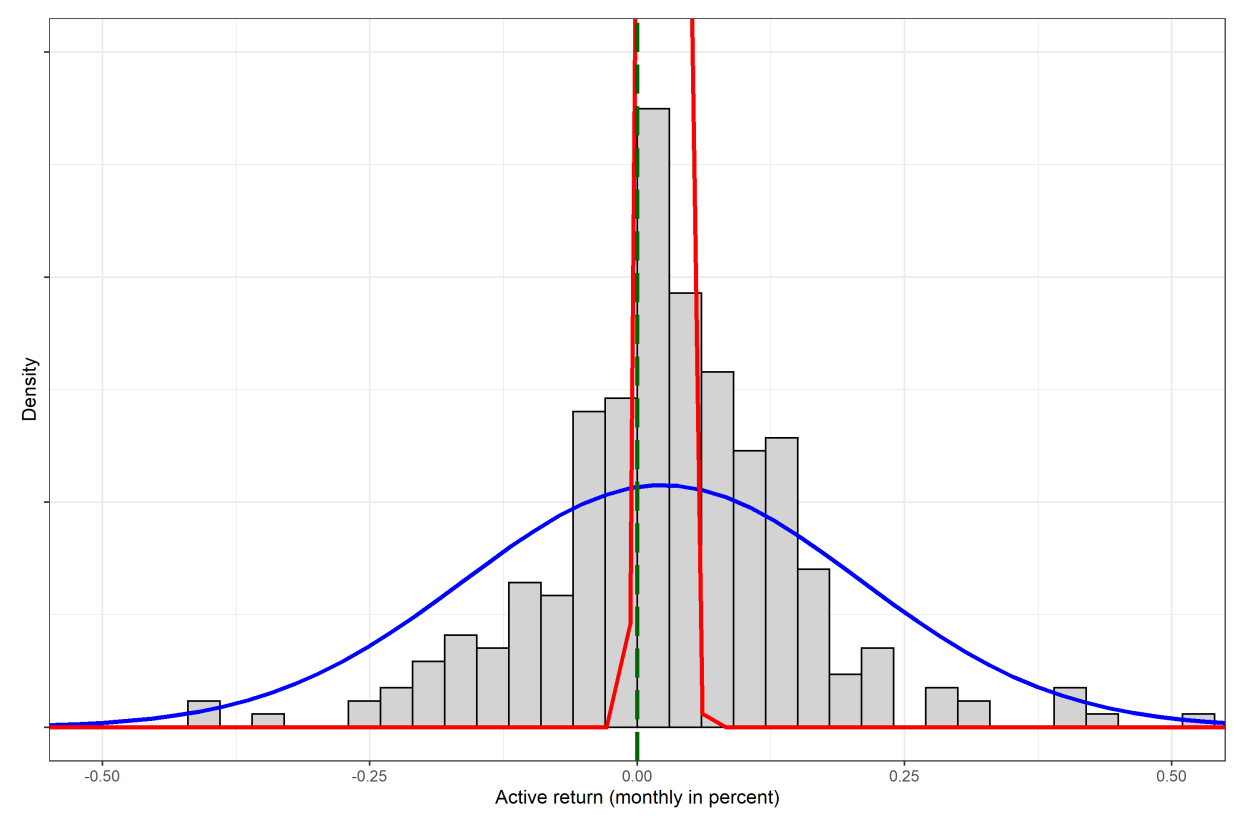

Figure 9: Histogram of the Fund's overall net active returns 
mean and standard deviation as the active returns. The red line is the density function with the same mean but with a standard deviation equal to the active returns' standard deviation divided by the square root of the number of observations, i.e., a proxy for the standard error. The main message, for now, is that the active return process fluctuates considerably. It is therefore hard to get enough power to reject the null hypothesis of no skill (mean zero).

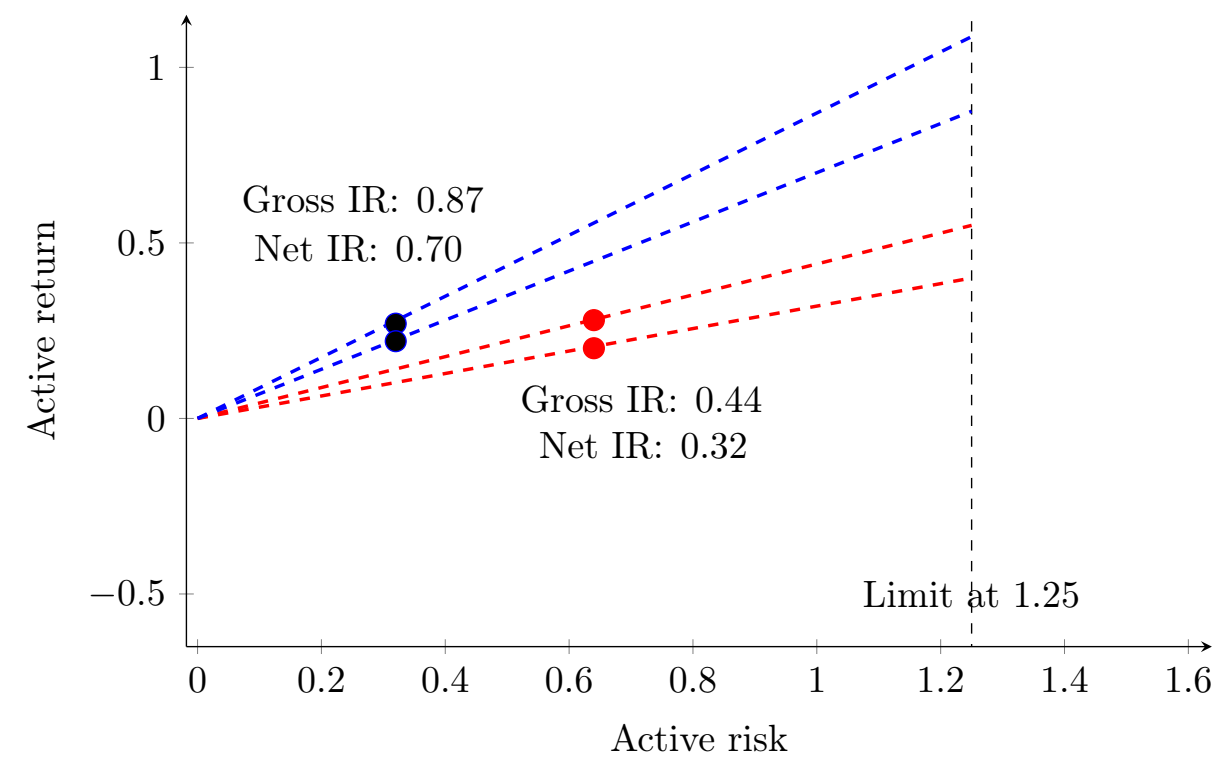

Realized active risk vs. ex-ante limit. Red: Jan 1998-Sep 2021, Blue: Jan 2017-Sep 2021.

Figure 10: Fund active return-active risk space

Figure 10 plots the average active return for the overall Fund portfolio and the tracking error for both the full (red lines) and recent (blue lines) sample periods. The tracking error for the recent period is lower than for the whole period for two main reasons. First, both the equity and fixed-income portfolios have had low volatility, making the tracking error low. Second, it might be that the actual active risk-taking of the Fund is lower now than it has been for the entire period.

Nevertheless, there is room for more active management up to the mandate's tracking-error limit of $1.25 \%$ annually. One reason for not exploiting the full risk capacity might be that NBIM is afraid of exceeding the limit, even only temporarily, thus holding a buffer for more volatile times. That is, the Fund takes the risk of extreme events into account. Another reason for the low risk taking might be that the individual portfolio managers are more risk averse than the limit set by the MoF. A final reason might be that the Fund has too few investment opportunities. The Fund is large, and even with a positive $I R$, higher value creation is dependent on a continuing positive $I R$. The scalability of active management is disputed in the academic literature (see, e.g., Berk and Green, 2004; Antón et al., 2021). We return to this discussion in Section 9.

\section{Separating skill from luck}

The goal of separating skill from luck is to identify whether NBIM has true skill that has created value that cannot be produced by simple, well-known strategies unskilled investors could implement. When we evaluate the returns, we neither know NBIM's original expectations nor whether those expectations made sense. We only observe the realized returns and hope that the noise in the outcomes does not obscure the evaluation of the NBIM's true underlying ability. For this reason, we use statistical analysis 
to help us separate skill from luck. Further, we assume that the drivers of returns have been the same over the sample period. Often, by the time it is possible to identify superior historic performance, a new "regime" has taken hold, such as a manager change or a recession period. However, we accommodate time variation in the performance of the Fund by investigating the recent subperiod separately.

In Table 4, we evaluate the mean active returns before and after costs for the full and the most recent sample period. The means are identical to those in Tables 2 and 3. All the mean active returns are positive. Here, we investigate the null hypothesis that the mean value is not significantly different from zero and the alternative hypothesis that it is. We adjust the standard errors for autocorrelation and heteroscedasticity using Newey and West (1987) standard errors. ${ }^{20}$

\begin{tabular}{lcc|cc}
\hline & \multicolumn{2}{c|}{ Full sample (1998-Sep 2021) } & \multicolumn{2}{c}{ Latest sample (2017-Sep 2021) } \\
\cline { 2 - 5 } & Gross & Net & Gross & Net \\
\hline Mean & 0.28 & 0.20 & $0.27^{*}$ & 0.22 \\
& $(0.22)$ & $(0.22)$ & $(0.14)$ & $(0.14)$ \\
$p$-value & .21 & .36 & .06 & .13 \\
$N$ & 285 & 285 & 57 & 57 \\
\hline
\end{tabular}

Notes: The table shows the gross and net mean annualized active returns in percent. Newey and West (1987) standard errors in parentheses. $N$ refers to the number of observations in the period. We denote by stars if the mean is significantly different from zero. ${ }^{* * * / * * / *}$ indicates significance at the $1 \% / 5 \% / 10 \%$ level.

Table 4: Fund mean active returns

In the table, stars indicate that the mean is significantly different from zero at a particular significance level $(1 \%, 5 \%$, or $10 \%)$. We provide the corresponding $p$-values so readers can form their own opinions. For the longest sample period, the mean active returns are not significantly different from zero at conventional significance levels (the $p$-values are .21 and .36). So, for the performance during the full sample period, we cannot reject the hypothesis that the Fund's performance is neutral. For the most recent period, the $p$-value for the gross active returns is .06 , while it is .13 for the net active returns. So, even with lower $p$-values, the performance in the recent period is still not significantly positive.

\subsection{Benchmark risk-adjusted performance}

In this section, we use CAPM to risk adjust the performance of the Fund using the Fund's benchmark in place of the market portfolio. Within this model, all differences in expected return are explained by the beta that measures the systematic risk of the portfolio. The model is estimated by regressing the portfolio excess returns on the benchmark excess returns:

$$
R_{t}-R_{f, t}=\alpha+\beta\left(R_{t}^{\mathrm{b}}-R_{f, t}\right)+\epsilon_{t}
$$

where $R_{\mathrm{f}, t}$ is a proxy for a risk-free rate and $\epsilon_{t}$ is the regression residual. The estimated slope coefficient is the beta of the Fund relative to its benchmark, and the estimated intercept (alpha) is the betaadjusted mean active return or the Fund's alpha after adjusting for the difference in beta risk between the Fund and its benchmark (Jensen's alpha). An alternative explanation is how much better the

\footnotetext{
${ }^{20}$ We have used the Newey-West function for R described at this page: https://search.r-project.org/CRAN/refmans/ sandwich/html/NeweyWest.html.
} 
Fund has performed relative to a combination of the benchmark portfolio and lending or borrowing at the risk-free interest rate where the combination has the same risk as the benchmark.

Table 5 presents the results of estimating Eq. (1) for the gross and net returns of the Fund's overall portfolio for the entire sample period and the most recent sample period. Although the data is monthly, we provide the annualized alphas for comparison with the previous analysis. We find that the estimated betas are above one (and significantly so). Since 2009, the Fund has been restricted from leveraging to increase its market exposure (see pp. 8 and 9 in the GICS report, NBIM, 2020i). Another reason for finding betas above one could be a result of choosing high-beta stocks instead of low-beta stocks. However, this is unlikely as we should not have positive alphas since low-beta stocks have performed well relative to their beta (see Frazzini and Pedersen, 2014; Hamaui and Jaffard, 2021).

The estimated alphas from Eq. (1) are lower than the mean active returns in Table 4. The interesting question is still the same: Are they significantly different from zero or not? For the whole sample, the gross and net annualized alpha is $0.14 \%$ and $0.06 \%$, respectively. The associated $p$-values are much larger than zero (.50 and .78), indicating that the alphas are not significant at any conventional significance level. For the most recent period, the gross and net annualized alphas are $0.19 \%$ and $0.14 \%$, respectively, with associated $p$-values of .13 and .27 . Therefore, we find that the benchmark risk-adjusted performance of the Fund's overall portfolio is not significantly different from its benchmark.

\begin{tabular}{lcc|cc}
\hline & \multicolumn{2}{c|}{ Full sample (1998-Sep 2021) } & \multicolumn{2}{c}{ Latest sample (2017-Sep 2021) } \\
\cline { 2 - 5 } & Gross & Net & Gross & Net \\
\hline$\alpha$ & 0.14 & 0.06 & 0.19 & 0.14 \\
& $(0.20)$ & $(0.21)$ & $(0.13)$ & $(0.13)$ \\
$p$-value & .50 & .78 & .13 & .27 \\
$\beta$ & $1.03^{* * *}$ & $1.03^{* * *}$ & $1.01^{* * *}$ & $1.01^{* * *}$ \\
& $(0.01)$ & $(0.01)$ & $(0.003)$ & $(0.003)$ \\
$p$-value & .01 & .01 & .01 & .01 \\
$N$ & 285 & 285 & 57 & 57 \\
$R^{2}$ & 1.00 & 1.00 & 1.00 & 1.00 \\
$A R$ & 0.24 & 0.10 & 0.64 & 0.47 \\
\hline
\end{tabular}

Notes: The table shows the estimated annualized $\alpha$ and $\beta$ from Eq. (1) using gross and net returns. Returns expressed in percent. Newey and West (1987) standard errors in parentheses. $R^{2}$ is the explanatory power from the regression, and $A R$ is the appraisal ratio. We denote by stars if $\alpha$ is significantly different from zero and if $\beta$ is significantly different from one. ${ }^{* * * / * * / *}$ indicates significance at the $1 \% / 5 \% / 10 \%$ level.

Table 5: Fund mean beta-adjusted active returns

Figure 10 shows that the portfolio's tracking error is lower than the MoF-mandated limit. We can back out a related measure of the degree of NBIM's active management from Eq. 1 . The $R^{2}$ from the regression measures how much of the variation of the Fund's excess returns $\left(R_{t}-R_{\mathrm{f}, t}\right)$ can be explained by the variation of the benchmark excess returns $\left(R_{t}^{\mathrm{b}}-R_{\mathrm{f}, t}\right)$. A number close to one indicates that the benchmark explains most of the variation and, thus, little active management. A number close to zero indicates that the benchmark explains almost none of the portfolio excess return and, thus, a high degree of active management. In all cases, the $R^{2}$ rounds to 1.00 . This result confirms that the benchmark's variation explains almost all of the Fund return's variation, suggesting little active 
management.

When we evaluate NBIM's active management, the appraisal ratio $(A R)$ is an appropriate measure. We find that $A R$ is positive for all four alternatives because the alpha estimates are positive. The $A R$ is higher for the recent sample period than for the entire sample period.

\subsection{Factor risk-adjusted performance}

So far, we have assumed that the Fund competes against the MoF's benchmark. The Fund's primary risk limit is the tracking-error limit. A more nuanced limit is that the MoF also has preferences concerning systematic risk. We find the following text in NBIM's mandate: "The equity portfolio and the fixed-income portfolio shall be sought composed in such a way that the expected excess return is exposed to several systematic risk factors" (Ministry of Finance, 2021, Section 2.4, paragraph 7). In a risk-management setting, documenting factor exposures against systematic risk is understandable, but its usefulness as a guideline for active management is not apparent to us. It is not clear ex ante how NBIM should be "punished" for taking factor risk. For example, which factors should be used? The MoF published no risk factors in NBIM's mandate. We think that as long as the Fund's tracking error is below the limit and the mandate only provides one strategic benchmark, the MoF should be cautious about punishing NBIM for taking on certain types of risk that can only be measured ex post. More follows on this topic in Section 9.2. This expression from Cochrane (2011, p. 1087) shed some light on this argument: "I tried telling a hedge fund manager, 'You don't have alpha. Your returns can be replicated with a value-growth, momentum, currency and term carry, and short-vol strategy.' He said, 'Exotic beta' is my alpha. I understand those systematic factors and know how to trade them. My clients don't." Thus, the dynamic exposures to the risk factors are part of the active management.

We consider performance analysis based on different versions of the Fama and French (2015) fivefactor model. Given the "zoo" of factors and factor models, we think that parsimonious versions of this model can still be viewed as the consensus model in empirical finance. ${ }^{21}$ This model is also recommended by NBIM's factor-model expert group (Dahlquist et al., 2015). Thus, NBIM might expect to be evaluated by it, although this is not explicitly stated by the MoF. The report recommends using a seven-factor model to evaluate the Fund, where the model is a combination of the global version of the Fama and French (2015) five-factor model and a fixed-income model with two factors, namely the term premium and the default factor. For the equity and fixed-income portfolios, we use restricted versions of the seven-factor model adapted to each asset class.

We use multivariate regressions of active returns before and after costs against sets of factor-return series. As the dependent variable, we use the active return. Thus, we investigate which factors explain the difference between the total portfolio returns and the benchmark. We interpret the estimated slope coefficients as the active exposures to the systematic factors over the sample period. This regression can be used to analyze the Fund's active investment style. For example, portfolios with positive exposure to the book-to-market factor are value portfolios, while negative exposures are growth portfolios. The

\footnotetext{
${ }^{21}$ The first factor model was the three-factor model of Fama and French (1992) and Fama and French (1993). The factors are chosen because the book-to-market ratio and company size measured by its market capitalization are important characteristics of a company's risk. Carhart (1997) adds a fourth factor, momentum, that captures persistence in equity returns. For an overview of the recent development in the factor literature, see, for example, Feng, Giglio and Xiu (2020), Harvey and Liu (2020), Hou, Mo, Xue and Zhang (2019), and Baltussen et al. (2021). Bartram, Lohre, Pope and Ranganathan (2021, Table 1) shows prominent asset pricing models.
} 
slope coefficient on each factor can be interpreted as the portfolio's implicit allocation to that factor. In this framework, we can interpret the regression intercepts as performance attributable to manager value creation over and above the exposure to the set of factors considered in the regression, and we denote the intercept as the alpha. ${ }^{22}$

We consider the seven-factor model

$$
\begin{aligned}
R_{t}^{\mathrm{a}}=\alpha+\beta_{M K T} M K T_{t}+\beta_{S M B} S M B_{t}+\beta_{H M L} H M L_{t} & +\beta_{R M W} R M W_{t} \\
& +\beta_{C M A} C M A_{t}+\beta_{T E R M} T E R M_{t}+\beta_{D E F} D E F_{t}+\epsilon_{t},
\end{aligned}
$$

where the factors are excess returns or zero-cost portfolios and $\epsilon_{t}$ is the regression residual. $M K T$ is the market excess return, $S M B$ is the small-minus-big portfolio, $H M L$ is the high-minus-low portfolio, $R M W$ is the robust-minus-weak portfolio, $C M A$ is the conservative-minus-aggressive portfolio, TERM is the term premium, and $D E F$ is the duration-adjusted default premium. More on the factors in Appendix B.

Factor models have many potential difficulties. First, we assume that the exposures (the betas) are constant over time. Our analysis estimates static exposures without picking up the dynamic changes in the factor exposures. ${ }^{23}$ Costs are associated with implementing the factor model's factor exposures (see Novy-Marx and Velikov, 2016; Li, Chow, Pickard and Garg, 2019). Achieving the desired exposures requires rebalancing the portfolio, which, in turn, induces trading costs. We assume that the exposures are reached without cost. Taking these costs into account would change the alpha estimates.

In addition, a recent study by Akey, Robertson and Simutin (2021) shows that using Fama and French (2015) data in factor models is not without measurement issues. The authors show that the factor returns differ substantially depending on when the data are collected, the data's vintage. The effects of these retroactive changes are large, even in the space of measuring active performance (alpha) in investment portfolios. In a mutual-fund context, they show that the annual alphas of almost half of the individual mutual funds change by more than one percentage point (could be in both directions). In this context, the potential "noise" resulting from the factors' vintage is much larger than a potential "signal" (the active return).

Another issue is the factor investability. The Fund might have restrictions such that it cannot replicate the factor portfolios. This can be due to short-selling restrictions, risk-budget constraints, maximum holdings in individual companies, and liquidity. NBIM's (2020a) analysis takes these restrictions into account, however, without seeing large effects on the estimated alphas. Finally, the return of the Fund benchmark differs from the market portfolio. Therefore, the Fund's estimated alpha can include an effect associated with the benchmark index, i.e., not satisfying the appropriate property discussed in Section 2.2.

Table 6 presents the results from estimating the factor model for the Fund's overall portfolio across the entire and recent sample periods. Even if the data and factor exposures are monthly, we provide the annualized alphas for comparison with the previous analysis. We find that alpha is still positive

\footnotetext{
${ }^{22}$ With a slight misuse of notation, we use alpha in both CAPM and the factor model.

${ }^{23}$ As commented by Dahlquist et al. (2015), taking time variation into account would require rich estimation techniques and is more suited for a stand-alone research paper. However, simple analysis based on Henriksson and Merton (1981) and Treynor and Mazuy (1966) could reveal, for example, timing with respect to the market.
} 


\begin{tabular}{|c|c|c|c|c|}
\hline & \multicolumn{2}{|c|}{ Full sample (1998-Sep 2021) } & \multicolumn{2}{|c|}{ Latest sample (2017-Sep 2021) } \\
\hline & Gross & Net & Gross & Net \\
\hline$\alpha$ & 0.14 & 0.06 & $0.38^{* * *}$ & $0.33^{* * *}$ \\
\hline & $(0.16)$ & $(0.16)$ & $(0.11)$ & $(0.11)$ \\
\hline$p$-value & .39 & .71 & .001 & .004 \\
\hline$M K T$ & $\begin{array}{c}0.01^{* * *} \\
(0.003)\end{array}$ & $\begin{array}{c}0.01^{* * *} \\
(0.003)\end{array}$ & $\begin{array}{c}0.001 \\
(0.003)\end{array}$ & $\begin{array}{c}0.001 \\
(0.003)\end{array}$ \\
\hline$p$-value & .0003 & .0003 & .79 & .79 \\
\hline$S M B$ & $\begin{array}{l}0.03^{* * *} \\
(0.01)\end{array}$ & $\begin{array}{l}0.03^{* * *} \\
(0.01)\end{array}$ & $\begin{array}{l}0.03^{* * *} \\
(0.01)\end{array}$ & $\begin{array}{l}0.03^{* * *} \\
(0.01)\end{array}$ \\
\hline$p$-value & .0000 & .0000 & .0001 & .0001 \\
\hline$H M L$ & $\begin{array}{l}0.01^{* *} \\
(0.01)\end{array}$ & $\begin{array}{l}0.01^{* *} \\
(0.01)\end{array}$ & $\begin{array}{c}0.01^{*} \\
(0.004)\end{array}$ & $\begin{array}{c}0.01^{*} \\
(0.004)\end{array}$ \\
\hline$p$-value & .04 & .05 & .07 & .07 \\
\hline$R M W$ & $\begin{array}{c}0.01 \\
(0.01)\end{array}$ & $\begin{array}{c}0.01 \\
(0.01)\end{array}$ & $\begin{array}{c}0.01 \\
(0.01)\end{array}$ & $\begin{array}{c}0.01 \\
(0.01)\end{array}$ \\
\hline$p$-value & .15 & .15 & .57 & .57 \\
\hline$C M A$ & $\begin{array}{c}-0.03^{* * *} \\
(0.01)\end{array}$ & $\begin{array}{c}-0.03^{* * *} \\
(0.01)\end{array}$ & $\begin{array}{c}0.003 \\
(0.01)\end{array}$ & $\begin{array}{c}0.003 \\
(0.01)\end{array}$ \\
\hline$p$-value & .01 & .01 & .71 & .71 \\
\hline TERM & $\begin{array}{c}-0.01^{* *} \\
(0.004)\end{array}$ & $\begin{array}{c}-0.01^{* *} \\
(0.004)\end{array}$ & $\begin{array}{c}-0.01 \\
(0.01)\end{array}$ & $\begin{array}{c}-0.01 \\
(0.01)\end{array}$ \\
\hline$p$-value & .02 & .02 & .34 & .34 \\
\hline$D E F$ & $\begin{array}{c}0.02^{*} \\
(0.01)\end{array}$ & $\begin{array}{r}0.02^{*} \\
(0.01)\end{array}$ & $\begin{array}{c}0.004 \\
(0.005)\end{array}$ & $\begin{array}{c}0.004 \\
(0.005)\end{array}$ \\
\hline$p$-value & .08 & .06 & .37 & .37 \\
\hline$N$ & 285 & 285 & 57 & 57 \\
\hline Adjusted $R^{2}$ & 0.47 & 0.47 & 0.46 & 0.46 \\
\hline
\end{tabular}

Notes: The table shows the estimated coefficients from the factor model in Eq. (2). $\alpha$ is annualized. Returns expressed in percent. Newey and West (1987) standard errors in parentheses. $N$ refers to the number of observations in the period, and $R^{2}$ is the explanatory power from the regression. We denote by stars if $\alpha$ or factors are significantly different from zero. ${ }^{* * * / * * / *}$ indicates significance at the $1 \% / 5 \% / 10 \%$ level.

Table 6: Fund factor model risk adjustment

for all specifications. The annualized alpha before (after) cost for the entire period is $0.14 \%(0.06 \%)$. Furthermore, we find that the associated $p$-values are large (.39 and .71). Interestingly, the alpha estimates are similar in size to the estimates from benchmark adjustments in Table 5 . This similarity suggests that adding the risk factors does not reduce the estimated alphas.

For the most recent period, the annualized gross and net alphas are $0.38 \%$ and $0.33 \%$, respectively. Interestingly, the alpha estimates are higher than the unadjusted mean active returns (see Table 2) and the benchmark risk-adjusted alphas (see Table 5). This finding suggests that the Fund has recently lost from its exposure to the additional factors. The alpha estimates have low $p$-value $(.001 \%$ and $.004 \%)$. Thus, the factor risk-adjusted performance is not significant for the full sample period, whereas it is significantly positive in the most recent sample period.

So far, we have only estimated the Fund's exposure to the factors and the corresponding alpha and betas. Table 7 quantifies how these factor exposures influence the factor-adjusted alpha. The Est column shows the beta estimates, and the Mean column shows the average return of the factors themselves. The Prod column, the product of the first two columns, shows how much the Fund has 
gained or lost from the factor exposure. Notice that these calculations use the estimated alpha and betas, even when they are not significantly different from zero.

\begin{tabular}{|c|c|c|c|c|c|c|}
\hline \multirow[b]{2}{*}{ Factor } & \multicolumn{3}{|c|}{ Full sample (1998-Sep 2021) } & \multicolumn{3}{|c|}{ Latest sample (2017-Sep 2021) } \\
\hline & Est & Mean & Prod & Est & Mean & Prod \\
\hline$M K T$ & 0.01 & 7.03 & 0.08 & 0.00 & 13.44 & 0.01 \\
\hline$S M B$ & 0.03 & 1.53 & 0.05 & 0.03 & -1.84 & -0.06 \\
\hline$H M L$ & 0.01 & 1.72 & 0.02 & 0.01 & -9.19 & -0.07 \\
\hline$R M W$ & 0.01 & 4.09 & 0.05 & 0.01 & 4.49 & 0.03 \\
\hline$C M A$ & -0.03 & 1.96 & -0.06 & 0.00 & -5.39 & -0.02 \\
\hline TERM & -0.01 & 3.43 & -0.03 & -0.01 & 2.94 & -0.02 \\
\hline$D E F$ & 0.02 & 1.76 & 0.04 & 0.00 & 4.38 & 0.02 \\
\hline Factor sum & & & 0.14 & & & -0.11 \\
\hline Net $\alpha$ & & & 0.06 & & & 0.33 \\
\hline Costs & & & 0.08 & & & 0.05 \\
\hline Gross mean & & & 0.28 & & & 0.27 \\
\hline
\end{tabular}

Notes: The table shows the factor exposures. The Est column shows the beta estimates, and the Mean column shows the factors' average return. The Prod column shows how much the Fund has gained or lost from the factor exposure, the product of the first two columns. Returns expressed in percent.

Table 7: Fund factor-exposure analysis

For the full sample, we find that the Fund has gained $0.08 \%$ for the overexposure to the market factor, while in total it has gained $0.14 \%$ from all the factors. When we sum the factor returns, the net alpha from Table 6, and the costs, we obtain the gross mean active return from Table 2.

Also, for the latest period, we can split the gross mean from Table 2 into the factor exposure, the net alpha, and the costs. Note that some of the factor means are negative in the latest period, unlike the entire sample period. For example, the average of $S M B$ and $H M L$ is negative. In this sample period, the Fund has lost from its factor exposures. The Fund has gained $0.01 \%$ on its overexposure to the market but lost due to $S M B$ and $H M L$. In sum, the Fund has lost $0.11 \%$ from its factor exposure. This also explains why the gross and net alphas from the factor regression in Table 6 are larger than the gross mean from Table 2. These findings might also help explain why the Fund has reduced its factor exposure strategies (see the latest strategy document in NBIM, 2021e).

\subsection{Summing up}

If the Fund's portfolio is found to generate positive and significant active returns using various approaches, the results are considered robust. The desire to have low correlation between the various sources of active returns is, among others things, documented in the fundamental law of active management (see Grinold and Kahn, 2000). The heat map in Figure 11 illustrates the average annualized active returns for each year in the sample period. If the average active return is positive, the square is a shade of green, while it is a shade of red for a negative return. During the 24 years in the sample period, the Fund has only five years with negative average active returns. That is a success (or hit ratio) of .79. The fixed-income portfolio has had seven years with negative average active returns, a success ratio of .71. For equity, the success ratio is .79. We find that the correlation between equity and fixed income is .27 based on monthly active returns. 


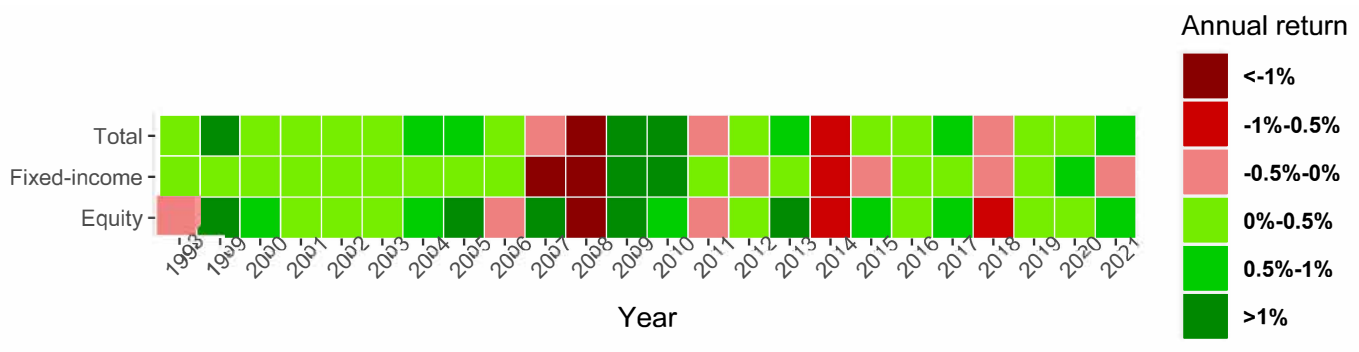

Figure 11: Heat map of gross active returns

Compared to the Fund's strategic benchmark, we have found that the Fund has earned NOK 170 billion after costs since 1998. The annualized average active return is $0.28 \%$ before costs and $0.20 \%$ after costs. Also, since 2017 these numbers have been positive. Most likely due to the noise in active returns, we cannot detect that these estimates are significantly different from zero. The same is true after risk adjusting. 


\section{Equity value creation}

We now examine the value creation from the Fund's equity portfolio. In this section, we start by analyzing the overall equity portfolio before we dig deeper into the different active equity strategies in Section 5. We follow the structure of the previous section and use the same empirical performance measures. We evaluate the performance of the Fund's equity portfolio without the part used for funding the real assets, i.e., the so-called managed portfolio. ${ }^{24}$

\subsection{Realized value creation}

Figure 12 plots the annualized value added from active management of the equity portfolio. We see that the variation of value added increases over time. This can be expected since value added amounts to the active returns weighted by the size of the Fund. The accumulated gross value added from 1998 is NOK 220 billion, calculated based on monthly AUM and active returns. The costs are NOK 43 billion, and then net value added is NOK 177 billion. For the recent sample period, the accumulated gross value added is NOK 135 billion, the accumulated costs are NOK 18 billion, and accumulated net value added is NOK 117 billion. In general, the value added has been positive in most years except for four years, the most recent of which is 2018 .

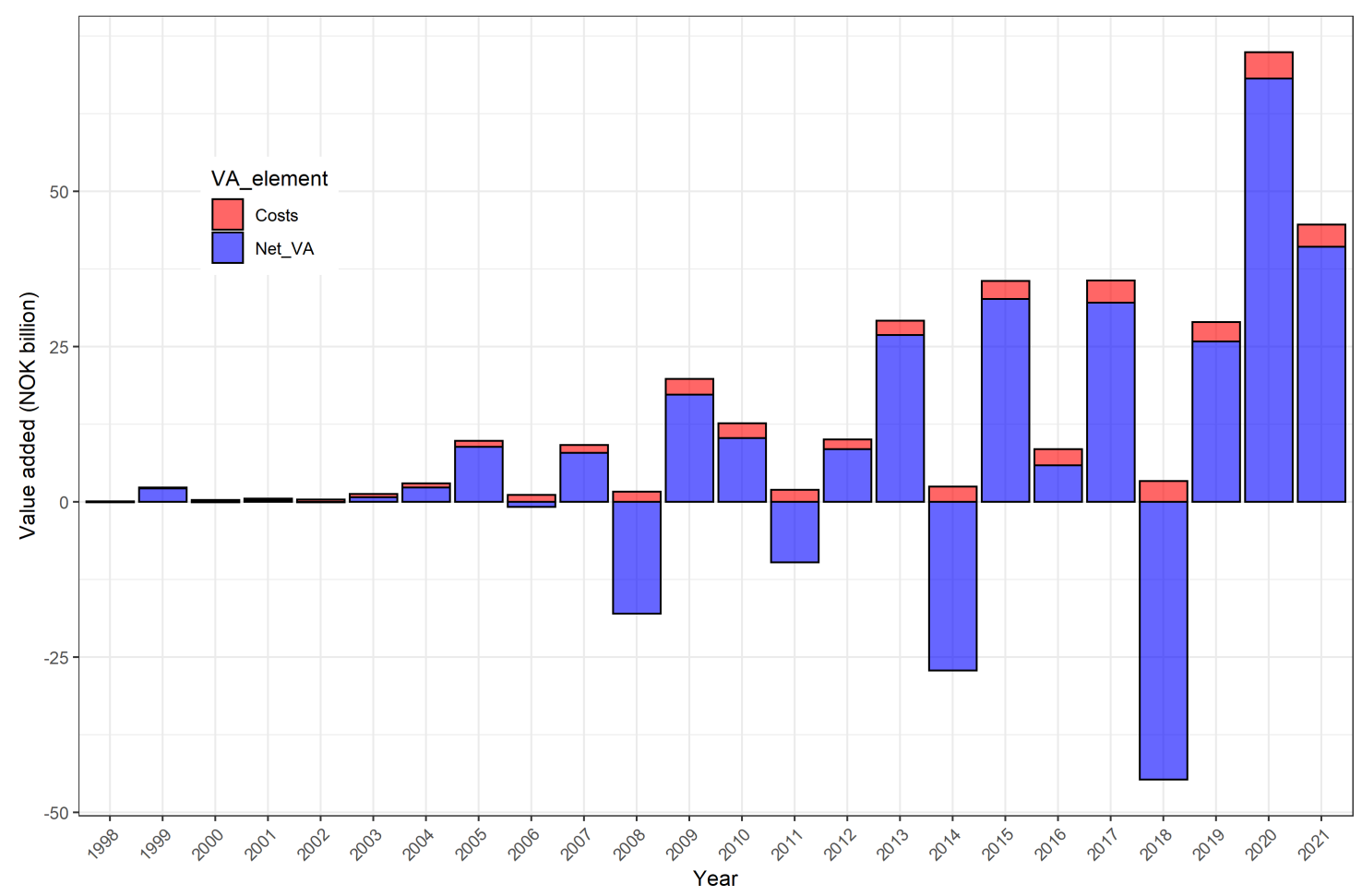

Figure 12: Equity gross and net value added

Figure 13 illustrates the development of the equity portfolio's active returns. A NOK 1,000 investment in the Fund's active-equity portfolio at its inception would have yielded NOK 1, 118 before and NOK 1,087 after costs compared to an investment in the benchmark. We see a relatively large decline during the financial crisis of 2008, but not as large as for the overall portfolio. The large downturn in total returns during the COVID crisis in March 2020 barely influenced the active returns.

\footnotetext{
${ }^{24}$ When we evaluate the total Fund in Section 2.4, we include the part funding real assets, i.e., the asset class portfolio.
} 


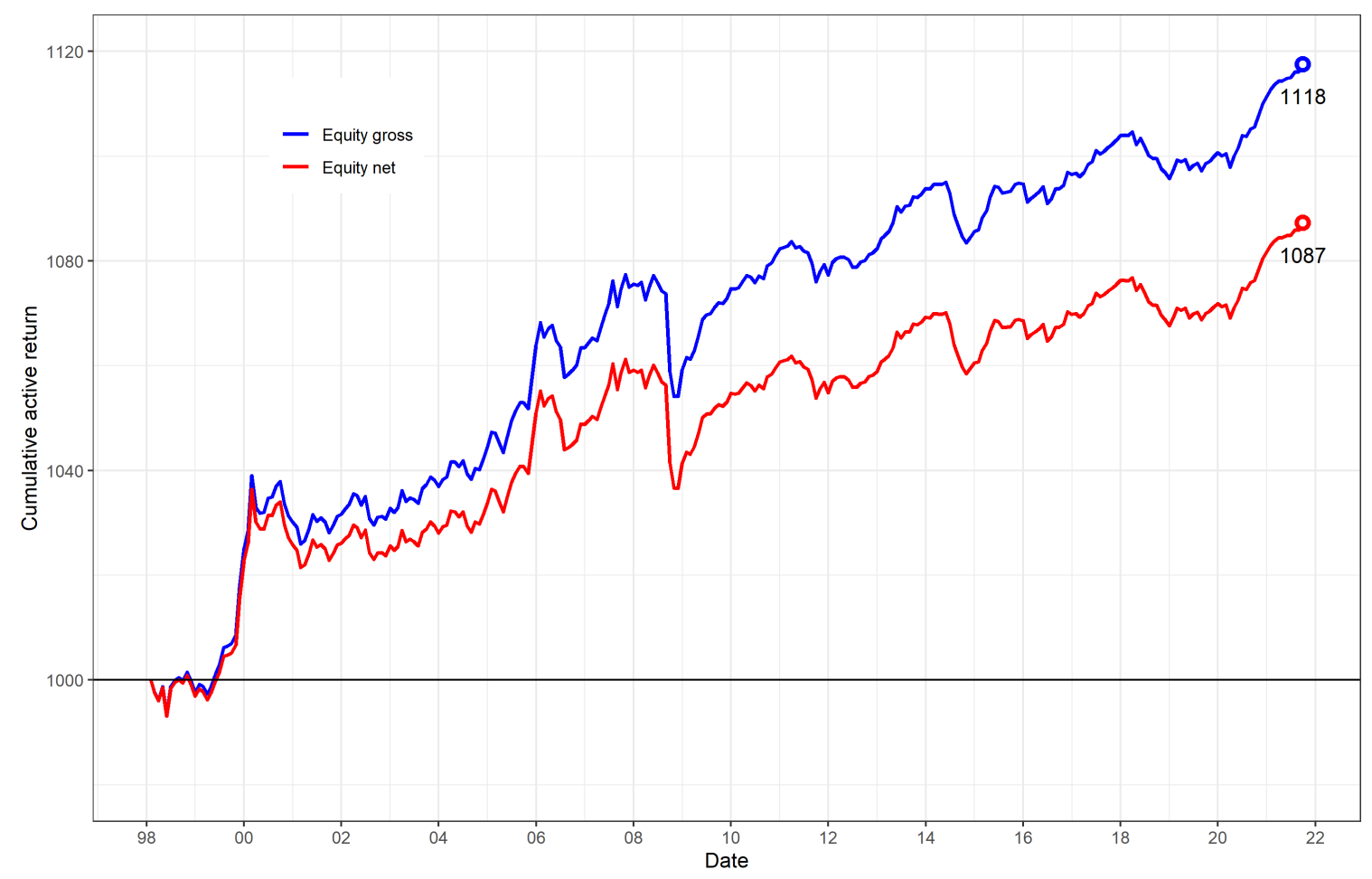

Figure 13: Equity cumulative gross and net active returns

Table 8 presents the monthly average active return and value added before and after costs. We find that the annualized mean active return is $0.47 \%$ before costs and $0.36 \%$ after costs. The monthly mean value added is NOK 0.77 billion before costs and NOK 0.62 billion after costs. This amount is similar to the Fund's overall value added. For the latest sample, the annualized mean active return is $0.40 \%$ before costs and $0.34 \%$ after costs. The value added is NOK 2.36 billion before costs and NOK 2.05 billion after costs.

\begin{tabular}{lcc|cc}
\hline & \multicolumn{2}{c|}{ Full sample (1998-Sep 2021) } & \multicolumn{2}{c}{ Latest sample (2017-Sep 2021) } \\
\cline { 2 - 5 } & Return & VA & Return & VA \\
\hline Gross & 0.47 & 0.77 & 0.40 & 2.36 \\
Costs & 0.12 & 0.15 & 0.06 & 0.31 \\
Net & 0.36 & 0.62 & 0.34 & 2.05 \\
\hline
\end{tabular}

Notes: The table shows the costs and the gross and net mean annualized active returns and monthly value added. Returns expressed in percent. Value added is in NOK billion.

Table 8: Equity realized active returns and value added

\subsection{Evaluation of active returns}

Table 43 in Appendix E shows the descriptive statistics for the active returns. From Figure 27 in Appendix E, we find that the active returns tend to be low for low benchmark returns. The histogram of the monthly active equity returns is in Figure 28. We see that the mass of the histogram is symmetric, but for equity even more mass is on the positive side than for the overall portfolio (Figure 9).

Figure 14 plots the active equity return and risk in the active return and active risk (tracking error) space. The equity portfolio does not come close to exploiting the tracking limit, and the active risk 
has decreased substantially in recent years.

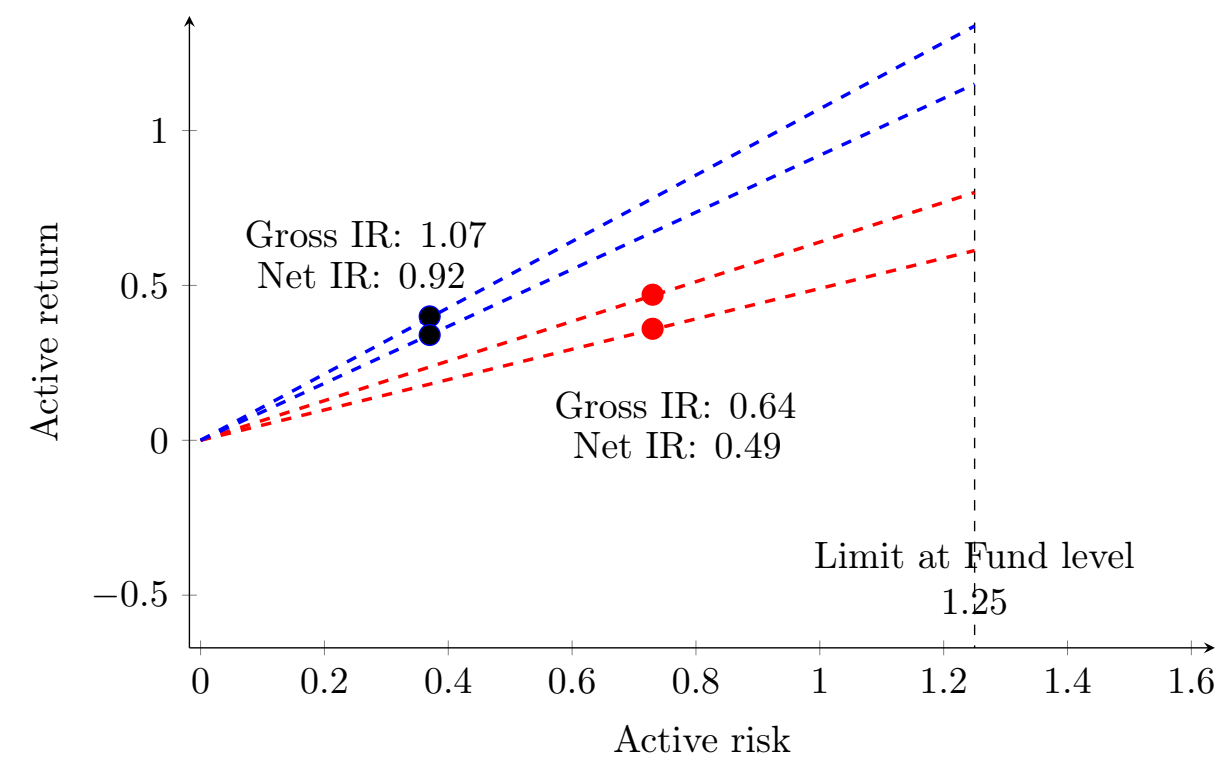

Realized active risk vs. ex ante limit. Red: Jan 1998-Sep 2021, Blue: Jan 2017-Sep 2021.

Figure 14: Equity active return-active risk space

Table 9 presents the mean active returns before and after cost for the full and most recent sample periods. We test whether the mean is different from zero. All the means in the table are positive and the associated $p$-values are at or below .10. Not surprisingly, the $p$-values are higher for net than for gross values. The annualized gross active return for the full sample period is $0.47 \%$ and it has a $p$-value of .01. Thus, we find that the gross active returns are significantly positive.

\begin{tabular}{lcc|cc}
\hline & \multicolumn{2}{c|}{ Full sample } & (1998-Sep 2021) & \multicolumn{2}{c}{ Latest sample } & (2017-Sep 2021) \\
\cline { 2 - 5 } & Gross & Net & Gross & Net \\
\hline Mean & $0.47^{* * *}$ & $0.36^{*}$ & $0.40^{* *}$ & $0.34^{*}$ \\
& $(0.18)$ & $(0.18)$ & $(0.20)$ & $(0.20)$ \\
$p$-value & .01 & .06 & .05 & .10 \\
$N$ & 284 & 284 & 57 & 57 \\
\hline
\end{tabular}

Notes: The table shows the estimated annualized mean active returns in percent. Newey and West (1987) standard errors in parentheses. $N$ refers to the number of observations in the period. We denote by stars if the mean is significantly different from zero. ${ }^{* * * / * * / *}$ indicates significance at the $1 \% / 5 \% / 10 \%$ level.

Table 9: Equity mean active returns

\subsection{Benchmark risk-adjusted performance}

Table 10 presents the annualized alphas before and after costs based on estimating Eq. (1) for the equity portfolio, the beta-adjusted mean active returns. Since the betas for all portfolios are greater than one and the Fund and benchmark returns are above the risk-free interest rate, the mean adjusted active returns are lower than without the adjustment. For the whole sample, the gross and net beta-adjusted annualized active returns are $0.37 \%$ and $0.25 \%$, respectively. The $p$-value before costs is .03 , while it is .13 after costs. For the most recent sample period, the gross and net beta-adjusted annualized active returns are $0.24 \%$ and $0.18 \%$, respectively, and the associated $p$-values are .09 and .20 . Overall, the beta-adjusted performance suggests that the performance of the equity portfolio is not all insignificant. 
For all periods and portfolios, the $R^{2}$ rounds to 1.00. This result confirms that the variation in the benchmark explains almost all the variation in the Fund's equity returns, which implies a very low degree of active equity risk. The $A R$ is also positive, for all four alternatives, but lower than $I R$ (shown in Table 43 in Appendix E).

\subsection{Factor risk-adjusted performance}

For the equity portfolio, we consider the following five-factor model. This is a reduced form (no fixed-income factors) of Eq. (2):

$$
R_{t}^{\mathrm{a}}=\alpha+\beta_{M K T} M K T_{t}+\beta_{S M B} S M B_{t}+\beta_{H M L} H M L_{t}+\beta_{R M W} R M W_{t}+\beta_{C M A} C M A_{t}+\epsilon_{t} .
$$

Table 11 presents the results from estimating the factor model for the equity portfolio for the full and the latest sample periods. The annualized alphas before and after cost for the entire period is $0.37 \%$ and $0.25 \%$, respectively. Furthermore, we find that the $p$-values are .02 and .11 , respectively. Surprisingly, we find that the size of the alpha estimates is similar to the estimates from benchmark adjustments in Table 10. This similarity suggests that the active returns do not disappear after adding the risk factors.

For the most recent period, gross and net annualized alphas are $0.31 \%$ and $0.25 \%$, respectively. These alpha estimates are higher than the corresponding benchmark-adjusted alphas from Table 10. When we adjust for factors for this sample, alpha estimates have a $p$-values of .04 and .09.

Table 12 quantifies how the factor exposures influence the factor-adjusted alphas. For the full sample, the equity managed portfolio has gained $0.09 \%$ from the overexposure to the market factor, while in total it has gained $0.11 \%$ from its factor exposures. Also, for the latest period, the Fund's equity portfolio has gained $0.10 \%$ from its overexposure to the market but has lost from its exposures to $S M B$ and $H M L$. In total, it has gained $0.09 \%$ from its factor exposures recently.

\begin{tabular}{lcc|cc}
\hline & \multicolumn{2}{c|}{ Full sample (1998-Sep 2021) } & \multicolumn{2}{c}{ Latest sample (2017-Sep 2021) } \\
\cline { 2 - 5 } & Gross & Net & Gross & Net \\
\hline$\alpha$ & $0.37^{* *}$ & 0.25 & $0.24^{*}$ & 0.18 \\
& $(0.16)$ & $(0.16)$ & $(0.14)$ & $(0.14)$ \\
$p$-value & .03 & .13 & .09 & .20 \\
$\beta$ & $1.02^{* * *}$ & $1.02^{* * *}$ & $1.01^{* * *}$ & $1.01^{* * *}$ \\
& $(0.004)$ & $(0.004)$ & $(0.002)$ & $(0.002)$ \\
$p$-value & .00 & .00 & .00 & .00 \\
$N$ & 284 & 284 & 57 & 57 \\
$R^{2}$ & 1.00 & 1.00 & 1.00 & 1.00 \\
$A R$ & 0.54 & 0.37 & 0.75 & 0.57 \\
\hline
\end{tabular}

Notes: The table shows the estimated $\alpha$ and $\beta$ from Eq. (1) for the gross and net returns in the full and latest sample periods. Returns expressed in percent. Newey and West (1987) standard errors in parentheses. $N$ is the number of observations in the sample period, $R^{2}$ is the explanatory power from the regression, and $A R$ is the appraisal ratio. We denote by stars if $\alpha$ is significantly different from zero and if $\beta$ is significantly different from one. ${ }^{* * * / * * / *}$ indicates significance at the $1 \% / 5 \% / 10 \%$ level.

Table 10: Equity mean beta-adjusted active returns 


\begin{tabular}{lcc|cc}
\hline & \multicolumn{2}{c|}{ Full sample $(1998-$ Sep 2021) } & \multicolumn{2}{c}{ Latest sample $(2017-$ Sep 2021) } \\
\cline { 2 - 3 } & Gross & Net & Gross & Net \\
\hline$\alpha$ & $0.37^{* *}$ & 0.25 & $0.31^{* *}$ & $0.25^{*}$ \\
$p$-value & $(0.15)$ & $(0.15)$ & $(0.15)$ & $(0.15)$ \\
$M K T$ & .02 & .11 & .04 & .09 \\
& $0.01^{* * *}$ & $0.01^{* * *}$ & $0.01^{* *}$ & $0.01^{* *}$ \\
$p$-value & $(0.003)$ & $(0.003)$ & $(0.003)$ & $(0.003)$ \\
$S M B$ & .0001 & .0001 & .02 & .02 \\
& $0.04^{* * *}$ & $0.04^{* * *}$ & $0.03^{* * *}$ & $0.03^{* * *}$ \\
$p$-value & $(0.01)$ & $(0.01)$ & $(0.01)$ & $(0.01)$ \\
$H M L$ & .00 & .00 & .0003 & .0003 \\
& -0.01 & -0.01 & $0.01^{* *}$ & $0.01^{* *}$ \\
$p$-value & $(0.01)$ & $(0.01)$ & $(0.01)$ & $(0.01)$ \\
$R M W$ & .51 & .48 & .04 & .04 \\
& 0.004 & 0.004 & 0.01 & 0.01 \\
$p$-value & $(0.01)$ & $(0.01)$ & $(0.01)$ & $(0.01)$ \\
$C M A$ & .70 & .70 & .45 & .45 \\
& $-0.03^{* * *}$ & $-0.03^{* * *}$ & -0.02 & -0.02 \\
$p$-value & $(0.01)$ & $(0.01)$ & $(0.01)$ & $(0.01)$ \\
$N$ & .01 & .01 & .11 & .11 \\
Adjusted $R^{2}$ & 284 & 284 & 57 & 57 \\
\hline
\end{tabular}

Notes: The table shows the estimated coefficients from the factor model in Eq. (3). $\alpha$ is annualized. Returns expressed in percent. Newey and West (1987) standard errors in parentheses. $N$ refers to the number of observations in the period, and $R^{2}$ is the explanatory power from the regression. We denote by stars if $\alpha$ or the factors are significantly different from zero. ${ }^{* * * / * * / *}$ indicates significance at the $1 \% / 5 \% / 10 \%$ level.

Table 11: Equity factor model risk adjustment

\begin{tabular}{lrrr|rrr}
\hline & \multicolumn{3}{c|}{ Full sample (1998-Sep 2021) } & \multicolumn{4}{c}{ Latest sample (2017-Sep 2021) } \\
\cline { 2 - 7 } Factor & Est & Mean & Prod & Est & Mean & Prod \\
\hline$M K T$ & 0.01 & 7.03 & 0.09 & 0.01 & 13.44 & 0.10 \\
$S M B$ & 0.04 & 1.53 & 0.06 & 0.03 & -1.84 & -0.06 \\
$H M L$ & -0.01 & 1.72 & -0.01 & 0.01 & -9.19 & -0.10 \\
$R M W$ & 0.00 & 4.09 & 0.02 & 0.01 & 4.49 & 0.04 \\
$C M A$ & -0.03 & 1.96 & -0.06 & -0.02 & -5.39 & 0.12 \\
\hline Factor sum & & & 0.11 & & & 0.09 \\
Net $\alpha$ & & & 0.25 & & & 0.25 \\
Costs & & & 0.12 & & & 0.06 \\
Gross mean & & & 0.47 & & & 0.40 \\
\hline
\end{tabular}

Notes: The table shows the factor exposures. The Est column shows the beta estimates, and the Mean column shows the average returns of the factors. The Prod column shows how much the Fund has gained or lost from the factor exposure, which is the product of the first two columns. Returns expressed in percent.

Table 12: Equity factor-exposure analysis 


\subsection{Summing up}

We document that the Fund's equity managed portfolio has earned NOK 177 billion after costs since 1998. The annual average active return is $0.47 \%$ before costs and $0.36 \%$ after costs. In a setting without risk adjustment, the Fund's equity portfolio has significantly positive gross active returns, while the net active returns are not significant. After risk adjusting, these results hold. 


\section{$5 \quad$ Equity strategies}

In this section, we dig deeper into the active equity-management process. We organize the assessment around the Fund's three equity substrategies, security selection, enhanced indexing, and fund allocation. ${ }^{25}$ According to NBIM, an argument for splitting the strategies into these three main groups is the time horizon of the bets. Enhanced indexing strategies have a shorter horizon than security selection, which is again shorter than fund allocation. Another reason for this organization is the separation of security selection and timing strategies. For security selection, the goal is to choose weights that are different from the benchmark, while strategies with timing characteristics are executed, along with also many other strategies, in the fund-allocation strategy. When discussing these strategies, we again rely heavily on the information and data from NBIM.

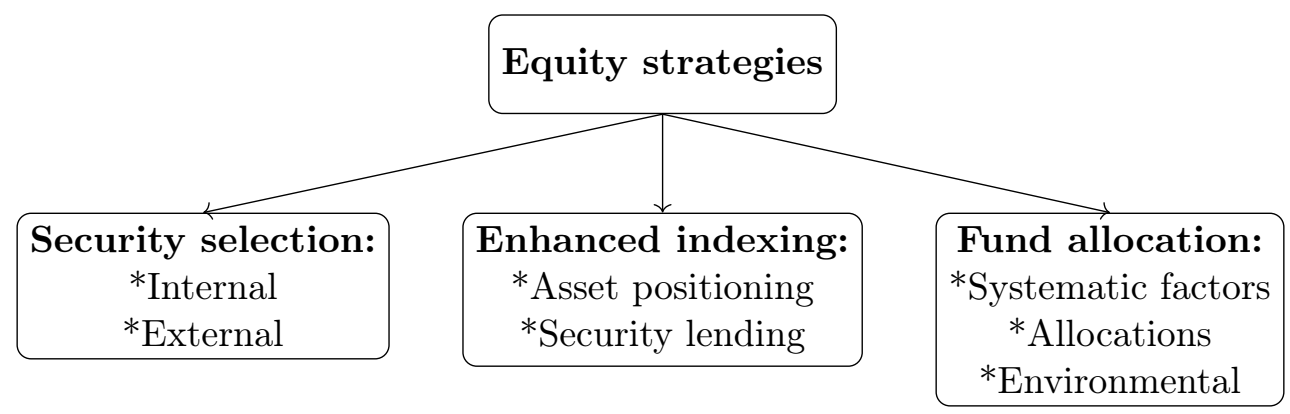

Figure 15: Equity substrategies

Within the three main equity strategies, there are also substrategies. Figure 15 outlines the different substrategies we examine. We faced a dilemma in choosing the suitable level of detail for reviewing the Fund's substrategies: the trade-off between heterogeneity within a given substrategy and complexity of too many strategies. For security selection, we think the strategies are fairly homogeneous and distinct: either internal or external security selection. For enhanced indexing, asset positioning and security lending are also fairly clearly different. For fund allocation, the strategies are more diverse. The environmental strategy is pure, but systematic factors and allocation decisions both constitute many different strategies and decisions. We return to this in the subsections below.

The substrategies are organized such that each portfolio manager is assigned an internal benchmark. NBIM has communicated to us that the constituents of these benchmarks come from the total equity benchmark, so the individual subbenchmarks add up to the equity benchmark. NBIM evaluates managers' actual portfolios relative to the internal benchmarks.

We have access to monthly returns for the different substrategies since the start of 2013. NBIM has communicated to us that the returns before 2013 are not available for the substrategies for various reasons. We will return to this in Section 9.3.

Table 44 in Appendix F, reports the mean returns, standard deviations, and Sharpe ratios for the total gross returns for the strategies and their benchmarks. Table 45 in the same appendix reports the same results in the Fund's currency basket.

As we can see from the total return-risk space in Figure 16, the benchmarks of the stock-selection strategies delivered lower total returns and higher risk than the overall equity benchmark. We suspect

\footnotetext{
${ }^{25}$ The Fund often names the enhanced indexing strategy for asset management. We think enhanced indexing covers best what is included in this strategy.
} 


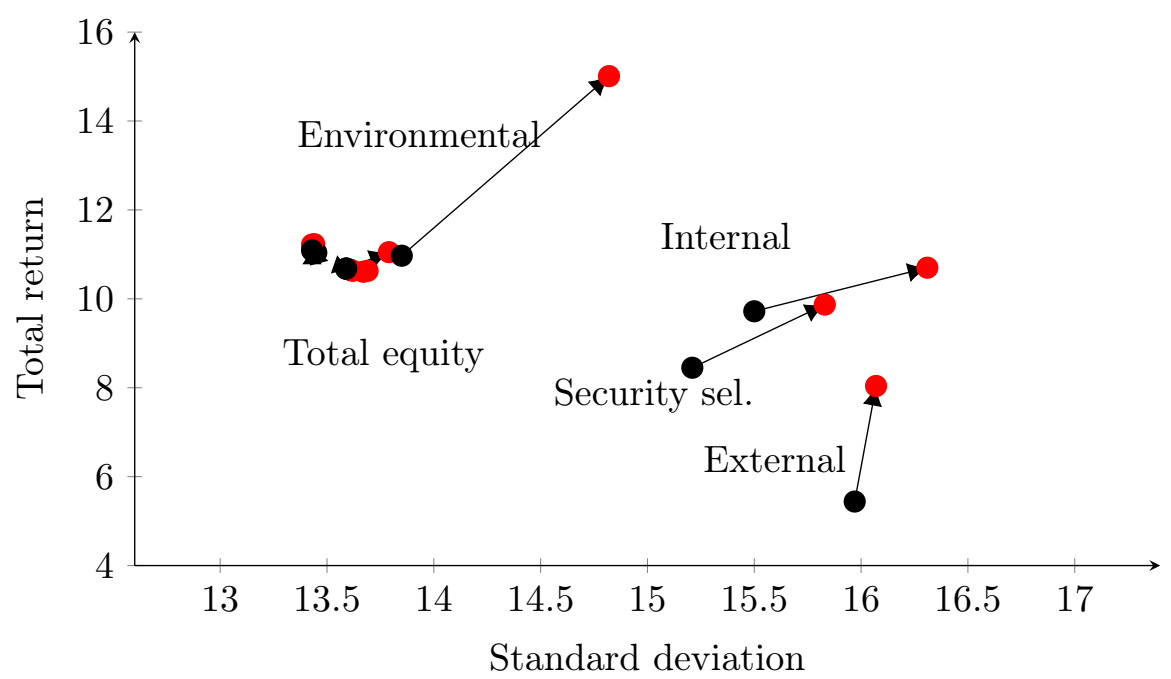

Realized total returns and risk of active and benchmark equity strategies. Benchmark portfolios in black and active portfolios in red.

Figure 16: Equity strategies in the total return-risk space

that this is because the part of the benchmark that involves a large degree of active management is the part with the lowest return. For example, it may be that a large part of the internal security selection has been in Europe, with a lower return relative to the total (and especially the US) portfolio.

A key question is how much capital is behind the individual substrategies. For example, the environmental strategy has performed well, but we also know that the associated AUM is relatively low. The AUM is a dynamic process, implying that the monthly data may include errors relative to the sum of the strategies. The strategies in security selection and enhanced indexing are fully funded with dedicated capital, except for the security lending substrategy. In the fund-allocation

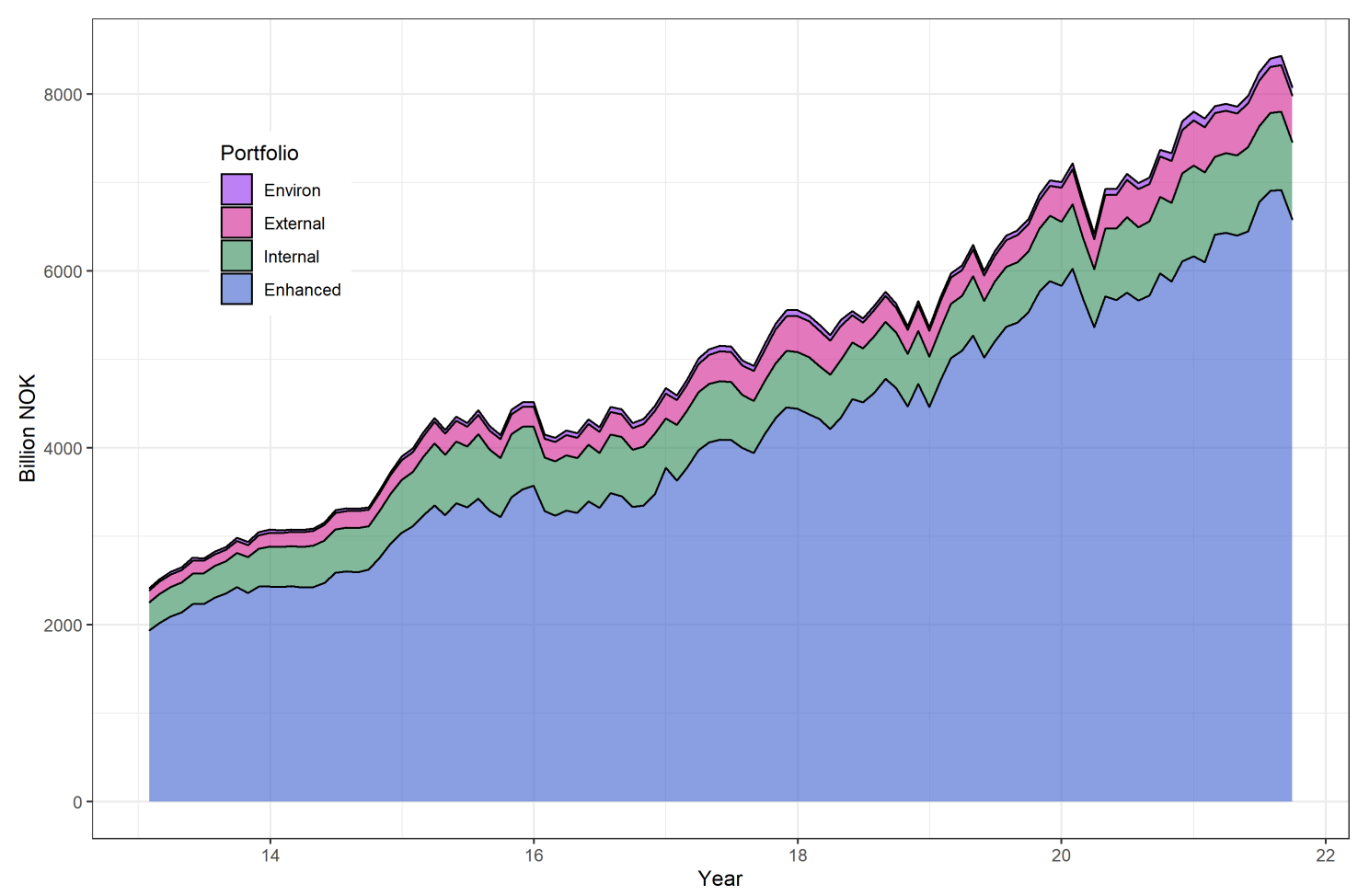

Figure 17: Equity strategies AUM 
strategy, however, the dominant sources of performance are overlay-type positions taken at the top management level before portfolios are assigned to dedicated mandates. A meaningful asset base for this strategy thus will also encompass the assets of the two other strategies. Hence, the AUM of the Fund's allocation strategy is equal to the total equity portfolio AUM. Figure 17 shows the growth of four large substrategies: internal security selection, external security selection, enhanced indexing, and environmental mandates. We see that most of the capital is managed in the enhanced-indexing strategy. Also, most of the growth of the equity portfolio comes from that portfolio, which suggests it might be hard to increase the scale in security selection.

Costs are also important in the evaluation of equity substrategies. We have already illustrated the total management costs for the equity portfolio in Figure 5 , but there might be differences in the costs of managing the respective substrategies. Again, we take NBIM's cost allocation to substrategies as a given. For security-selection analysis, we report value creation before and after costs. NBIM has not provided us with costs allocated into equity and fixed income for substrategies, so we use the average costs for internal and total security selection for both asset classes. For external security selection, we use the costs for equity because there is no external fixed-income security-selection strategy. Costs are smaller for the other substrategies due to both lower total costs and the AUM magnitude. Hence, we only consider gross value creation for these strategies.

Before we examine each of the substrategies in detail, we investigate the correlations between the substrategies. Figure 18 shows the unconditional correlation matrix of active returns. Overall, the correlations are quite low. For example, the asset-positioning strategy is weakly correlated with internal $(-.11)$ and external security selection $(-.06)$. Not surprisingly, security lending is weakly correlated with other substrategies. This is reassuring for NBIM; the low correlations suggest the substrategies are not competing for the same profit opportunities. However, be aware that the investigated sample period has few large negative shocks such as recession or crisis periods.

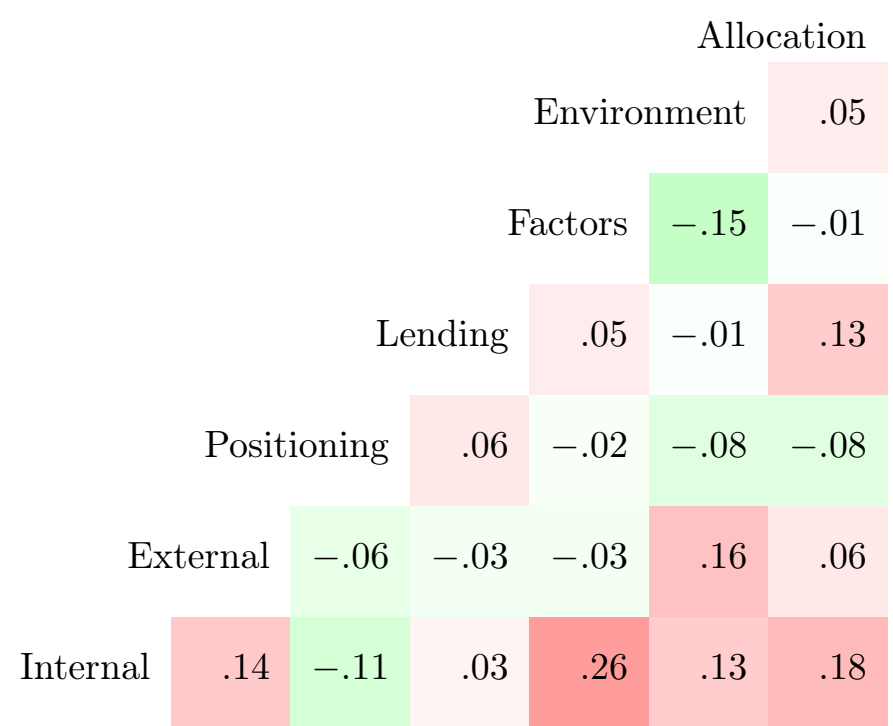

Unconditional active-return correlation matrix, green background if negative correlation and red if positive (in contrast to the returns reported in Section 9). Sample: 2013-2021.

Figure 18: Equity strategies correlation matrix 


\subsection{Security selection}

NBIM has organized its security selection strategies into two main substrategies, internal and external mandates.

\section{Internal mandates}

NBIM (2020g) describes the internal active equity management. As a long-term investor, NBIM is particularly well-positioned to make investments based on developments that will not necessarily become evident in the short run. However, such improvements are not always easy to identify. NBIM, therefore, spends considerable resources on researching companies, the markets in which they operate, and the issues they face. NBIM meets with companies they invest in, typically with senior management. Furthermore, NBIM claims that the company dialogue improves their understanding of the companies and their situation. It also builds strong relationships to support and complement the active-ownership strategies. We return to this in Section 9.

We can also learn from NBIM's own description of the security selection: "The investment strategy for the Fund was based on diversification. A large number of internal and external concentrated and specialized mandates have been the hallmark of our investment strategy since inception. We wanted a set of different investment strategies, and we wanted the majority of these to have a variety of investment positions. The different investment styles and approaches should all be based on specialist expertise. These were the elements of the fundamental law of active management (Grinold, 1989). We sought to build adequate skill to achieve outperformance, and a structure with the breadth of many independent investment positions. The company insight strategies sought to develop investment skill through fundamental company insight. The breadth would come from numerous and independent investment mandates" (see NBIM, 2020g, p. 29).

The individual portfolio managers who engage with the companies make most of the investment decisions. Individual investment mandates for particular subportfolios of the Fund specify these responsibilities. The active internal-security-selection management consists of four central mandates. NBIM (2020g, Table 4) shows the AUM for the different mandate groups, Table 2 shows their active returns each year, and Chart 50 shows each mandate's performance. We will not describe these mandates in detail.

\section{External mandates}

NBIM (2020h) describes external active equity-selection management. In this setting, NBIM is an investor in that NBIM outsources the investment decisions (see Sharpe, 1981; van Binsbergen, Brandt and Koijen, 2008; Gerakos et al., 2021, for discussions). Gârleanu and Pedersen (2018) show theoretically how an investor with low search costs, such as NBIM, can capture economic rents by identifying informed delegated asset managers. The active decisions then become part of a multistage process that first allocates capital to different substrategies and then makes active investments within the given universe. NBIM has built expertise in selecting external managers who specialize in assessing the return potential of individual companies. Mainly in emerging markets, the Fund invests in equity through locally based external managers. It seems sensible to pursue active management in less efficient 
markets, see, for example, Dyck et al. (2013) in a pension fund context and Dyakov, Jiang and Verbeek (2019) in a mutual fund context. Investment in these markets benefit from market-specific knowledge because public transparency and corporate governance standards vary considerably there.

\section{Results}

Reinspection of Figure 16 shows that all three security-selection benchmarks (total, internal, and external) have lower returns and higher risk than the total equity benchmark. As discussed before, this may be because of a geographic or industry focus. If the internal security team mainly takes active positions on European stocks or particular industries, then the total returns of this portfolio may not correspond with the total equity benchmark.

The accumulated gross value added from 2013 is NOK 130 billion in total, NOK 64 billion for internal, and NOK 66 billion for external security selection. The corresponding accumulated costs are NOK 12 billion, NOK 3 billion, and NOK 11 billion, ${ }^{26}$ and the accumulated net values added are NOK 119 billion, NOK 61 billion, and NOK 54 billion. Thus, the two strategies have created about the same value added, despite the fact that the external mandates have substantially lower AUM.

Table 13 presents the monthly average active return and value added before and after costs for the security-selection strategy. We find that the annualized mean active return is $1.42 \%$ before costs and $1.27 \%$ after costs for the total security-selection strategy. The mean monthly value added is NOK 1.24 billion before costs and NOK 1.13 billion after costs. The annualized mean active return for internal stock selection is $0.98 \%$ before costs and $0.93 \%$ after costs. The gross value added is NOK 0.61 billion before costs and NOK 0.58 billion after costs. The annualized mean active return for external stock selection is $2.60 \%$ before costs and $2.15 \%$ after costs. The gross value added is NOK 0.63 billion before costs and NOK 0.52 billion after costs. So, external security selection has higher active returns and higher costs than internal security selection.

\begin{tabular}{lcc|cc|cc}
\hline & \multicolumn{2}{c|}{ Sec. select } & \multicolumn{2}{c|}{ Internal } & \multicolumn{2}{c}{ External } \\
\cline { 2 - 7 } & Return & VA & Return & VA & Return & VA \\
\hline Gross & 1.42 & 1.24 & 0.98 & 0.61 & 2.60 & 0.63 \\
Costs & 0.15 & 0.11 & 0.06 & 0.03 & 0.45 & 0.11 \\
Net & 1.27 & 1.13 & 0.93 & 0.58 & 2.15 & 0.52 \\
\hline
\end{tabular}

Notes: The table shows the mean annualized active returns and monthly value added. Returns expressed in percent. Value added is in NOK billion. Sample period: 2013-2021.

Table 13: Equity-security-selection realized active returns

Table 46 in Appendix F presents descriptive statistics of security selection's gross and net returns. Because all the active portfolios have positive active returns, $I R$ is also positive. Note that security selection's tracking errors are quite low, just below $2 \%$. This is considerably lower than we would expect from, for example, an active mutual fund (see Cremers and Petajisto, 2009; Cremers et al., 2019). As expected, the tracking error of the combined portfolio is lower than the average of the two, confirming the diversification benefits identified in Figure 18.

\footnotetext{
${ }^{26}$ Notice that these costs do not add up. The reason is that we only have the average costs for both equity and fixed income. With just one substrategy in fixed income, internal management, we estimate that the total cost should be about NOK 3 billion more for all equity strategies.
} 
Table 14 presents the mean net active returns and the beta-adjusted active returns (net alpha) based on estimating Eq. (1) for the security-selection strategies. At the total equity-security-selection level, $p=.04$ before beta adjusting and .10 after beta adjusting. For the internal security selection, the average annualized active return is $0.93 \%$, which is not significantly different from zero ( $p$-value of .20). The beta-adjusted active return (Jensen's alpha) is also positive at $0.50 \%$, but not significant ( $p$-value of .51). The results for the external-security-selection strategy are very different. Both the average active return and alpha are positive and high: $2.15 \%$ and statistically significant, with $p$-values close to zero.

The internal-security-selection strategy has a relatively high beta, while the external beta is about one. As already discussed at the Fund portfolio level, the high beta may be due to leveraging up or simply substituting low-beta stocks with high-beta stocks. We do not have enough data to conclude whether this exposure is planned or occurs by chance. The $A R$ is positive for both internal and external security selection but lower than the IR. In Table 47 in Appendix F, we report the same analysis before costs.

\begin{tabular}{lcc|cc|cc}
\hline & \multicolumn{2}{c|}{ Security selection } & \multicolumn{2}{c|}{ Internal } & \multicolumn{2}{c}{ External } \\
\cline { 2 - 7 } & Mean & Beta adjustment & Mean & Beta adjustment & Mean & Beta adjustment \\
\hline$\alpha$ & $1.27^{* *}$ & $0.98^{*}$ & 0.93 & 0.50 & $2.15^{* * *}$ & $2.15^{* * *}$ \\
$p$-value & $(0.61)$ & $(0.60)$ & $(0.72)$ & $(0.76)$ & $(0.63)$ & $(0.64)$ \\
$\beta$ & .04 & .10 & .20 & .51 & .001 & .001 \\
& & $1.04^{* * *}$ & & $1.05^{* * *}$ & & 1.00 \\
$p$-value & & $(0.01)$ & & $(0.01)$ & & $(0.02)$ \\
$N$ & .00 & & .00 & & .97 \\
Adjusted $R^{2}$ & 105 & 105 & 105 & 105 & 105 & 105 \\
$A R$ & & 0.99 & & 0.99 & & 0.99 \\
\hline
\end{tabular}

Notes: The table shows the mean active return in the Mean column and the estimated annualized $\alpha$ and $\beta$ from Eq. (1) in the Beta adjustment column. The analysis is based on net returns. Returns expressed in percent. Newey and West (1987) standard errors in parentheses. $R^{2}$ is the explanatory power from the regression, and $A R$ is the appraisal ratio. We denote by stars if $\alpha$ is significantly different from zero and if $\beta$ is significantly different from one. ${ }^{* * * / * * / *}$ indicates significance at the $1 \% / 5 \% / 10 \%$ level.

Table 14: Equity-security-selection mean active net returns with and without beta adjusting

We estimate the five-factor model from Eq.(3) for the total-, internal-, and external-security-selection strategies. Table 48 in Appendix F presents the estimation results. The factor risk-adjusted alphas are positive but insignificant for the internal strategy ( $p$-value of .53) and significant for the external strategy ( $p$-value of .00). Table 15 shows the factor exposures. For internal (external) stock selection, we find that the gross mean return of $0.98 \%(2.60 \%)$ can be split into $0.49 \%(0.55 \%)$ from factor exposures and $0.43 \%$ (1.60\%) from net alpha. Furthermore, we find that the main part of the factor return is due to overexposure to the market factor and negative exposure to the $C M A$ factor.

We are aware that most of the internal equity management occurs in Europe. We have also been informed that most of the external security selection involves emerging markets. We could add further factors controlling for these types of exposure to the factor model. ${ }^{27}$ We come back to the choice of the appropriate factor model in Section 9.2.

\footnotetext{
${ }^{27}$ We have also seen in NBIM's internal monthly risk and performance reports that they estimate their exposures to the same factors as we do and further quality factors, momentum, and liquidity.
} 


\begin{tabular}{lrrr|rr|rr}
\hline & & \multicolumn{2}{c|}{ Security selection } & \multicolumn{2}{c|}{ Internal } & \multicolumn{2}{c}{ External } \\
\cline { 3 - 8 } Factor & Mean & Est & Prod & Est & Prod & Est & Prod \\
\hline$M K T$ & 11.65 & 0.04 & 0.45 & 0.05 & 0.54 & 0.02 & 0.26 \\
$S M B$ & -0.62 & 0.05 & -0.03 & 0.04 & -0.03 & 0.07 & -0.04 \\
$H M L$ & -4.62 & 0.01 & -0.06 & 0.02 & -0.11 & -0.02 & 0.11 \\
$R M W$ & 3.39 & -0.04 & -0.14 & -0.07 & -0.22 & -0.01 & -0.02 \\
$C M A$ & -2.39 & -0.13 & 0.31 & -0.13 & 0.32 & -0.10 & 0.25 \\
\hline Factor sum & & & 0.53 & & 0.49 & & 0.55 \\
Net $\alpha$ & & & 0.74 & & 0.43 & & 1.60 \\
Cost & & 0.15 & & 0.06 & & 0.45 \\
Gross mean & & & 1.42 & & 0.98 & & 2.60 \\
\hline
\end{tabular}

Notes: The table shows the factor exposures. The Est column shows the beta estimates, and the Mean column shows the average return of the factors. The Prod column shows how much the Fund has gained or lost from the factor exposure, which is the product of the first two columns. Returns expressed in percent.

Table 15: Equity-security-selection factor-exposure analysis

\subsection{Enhanced indexing}

As described in NBIM (2020c), enhanced indexing consists of two main strategies: asset positioning and security lending.

\section{Asset positioning}

NBIM (2020c, Section 2) describes asset positioning. The message is that the extensive diversified global holdings enable NBIM to create returns by exploiting market-price differences in securities with similar characteristics. Other market participants' constraints or preferences often drive these price differences. Providing liquidity to less patient investors at a price is part of this work to capitalize on the Fund's security holdings. The relative value strategies are an integrated part of the work to maintain the Fund's desired market exposures.

NBIM tries to minimize and control transaction costs that come with implementing these investment strategies and to avoid the weaknesses of mechanical benchmark replication. These often lead to higher friction costs, particularly for large funds. NBIM also states that they seek to be patient in portfoliorebalancing decisions, using natural liquidity and capital-market events to implement longer-term exposure targets. Where possible, NBIM tries to benefit from the behavior and liquidity profile of other, more constrained market participants. In the end, the performance evaluation of this substrategy will show whether NBIM is successful in profiting from this strength.

\section{Security lending}

NBIM (2020c, Section 3) describes security lending. NBIM uses both direct internal security lending and external agency security lending through a custodian. Security lending exposes the Fund to counter-party default risk. NBIM manages this risk by securing all security-lending transactions with appropriate collateral and by diversifying exposures to different counter-parties. 


\section{Results}

Since 2013, the accumulated gross value added stemming from equity enhanced indexing is NOK 66 billion, of which NOK 45 billion can be attributed to asset positioning, and NOK 23 billion to security lending.

Table 16 shows that the average annualized active return from these strategies is $0.20 \%$. While this is a relatively small number, the average monthly value added is about as large as the value added from either the internal or external-security-selection strategy. Table 49 in Appendix F shows summary statistics for the gross returns. Interestingly, this strategy's hit ratios are very high. We discuss this in Section 9.1.

\begin{tabular}{ccc|cc|cc}
\hline & \multicolumn{2}{c|}{ Enhanced } & \multicolumn{2}{c|}{ Positioning } & \multicolumn{2}{c}{ Lending } \\
\cline { 2 - 7 } & Return & VA & Return & VA & Return & VA \\
\hline Gross & 0.20 & 0.62 & 0.13 & 0.42 & 0.07 & 0.21 \\
\hline
\end{tabular}

Notes: The table shows the mean annualized active returns and monthly value added. Returns expressed in percent. Value added is in NOK billion. Sample period: 2013-2021.

Table 16: Equity-enhanced-indexing realized active return

Table 17 shows the mean and the beta-adjusted active returns. In all cases, the active returns and alphas are positive and significant ( $p$-values close to zero) due to the low standard deviation of this strategy. Tables 50 and 51 in Appendix F show that the factor exposures from these strategies are almost zero and that the factor risk-adjusted alphas are significantly positive. Thus, for the given period, the active returns do not come from factor exposures.

\begin{tabular}{lcc|cc|cc}
\hline & \multicolumn{2}{c|}{ Enhanced } & \multicolumn{2}{c|}{ Positioning } & \multicolumn{2}{c}{ Lending } \\
\cline { 2 - 6 } & Mean & Beta adjust. & Mean & Beta adjust. & Mean & Beta adjust. \\
\hline$\alpha$ & $0.20^{* * *}$ & $0.21^{* * *}$ & $0.13^{* * *}$ & $0.14^{* * *}$ & $0.07^{* * *}$ & $0.07^{* * *}$ \\
$p$-value & $(0.05)$ & $(0.05)$ & $(0.04)$ & $(0.04)$ & $(0.01)$ & $(0.005)$ \\
$\beta$ & .0001 & .0001 & .002 & .002 & .00 & .00 \\
& & 1.00 & & 1.00 & & 1.00 \\
$p$-value & & $(0.00)$ & & $(0.00)$ & & $(0.00)$ \\
$N$ & & .28 & & .52 & & .57 \\
Adjusted $R^{2}$ & 105 & 105 & 105 & 105 & 105 & 105 \\
$A R$ & & 1.00 & & 1.00 & & 1.00 \\
\hline
\end{tabular}

Notes: The table shows the mean annualized active return in the Mean column and the estimated annualized $\alpha$ and $\beta$ from Eq. (1) in the Beta adjustment column. Returns expressed in percent. Newey and West (1987) standard errors in parentheses. $N$ refers to the number of observations in the period, $R^{2}$ is the explanatory power from the regression, and $A R$ is the appraisal ratio. We denote by stars if $\alpha$ is significantly different from zero and if $\beta$ is significantly different from one. ${ }^{* * * / * * / *}$ indicates significance at the $1 \% / 5 \% / 10 \%$ level.

Table 17: Equity-enhanced-indexing mean active returns with and without beta adjusting

\subsection{Fund allocation}

Equity-fund allocation strategies include three substrategies: systematic factors, environmental mandates, and allocation decisions. These strategies are harder to describe as NBIM does not publish a 
detailed explanation. The environmental mandate is a compulsory strategy for NBIM as it is explicitly included in its mandate. Thus, NBIM is forced to integrate this active position in its investment strategy. We think it might have been more natural to organize and document it under security-selection strategies. We will discuss this further in Section 9.3. The two other substrategies (systematic factors and allocation decisions) have a top-down nature and fit well in this category.

\section{Systematic factors}

Both Ang et al. (2009) and Ang et al. (2014) highlighted that NBIM is well positioned to make investments to harvest risk-factor premia. Shortly after their publication, NBIM began managing dynamic factor strategies. From NBIM (2021e) and our conversations with NBIM, we conclude that they are currently phasing out factor strategies, presumably, as we see below, due to the strategies' poor performance.

\section{Environmental mandates}

NBIM is mandated to invest NOK 30-120 billion in environment-related investments (Ministry of Finance, 2021, Section 4.4). We return with a discussion hereof in Section 9. Still, we already flag here that we agree with the climate-risk committee that this creates unclear responsibilities going forward (Skancke et al., 2021).

\section{Allocation decisions}

We have trouble separating the allocation decisions from systematic factor strategies. However, we understand that NBIM used to make allocation decisions, which gave access to a broader investment universe. NBIM defined a so-called internal reference portfolio, which served as a starting point for the Fund's investments. NBIM sought to improve diversification by adding additional markets and asset classes not part of the strategic benchmark. The objective of the internal reference portfolio has been to obtain the best possible long-term return-risk profile for the Fund within the opportunity set defined in the mandate. Based on our conversations and interviews with NBIM, we conjecture that allocation-decision strategies have diminished over time (see NBIM, 2021e).

\section{Results}

Since 2013, the three fund-allocation strategies have jointly yielded an accumulated gross value added of NOK -16 billion. Table 18 shows that systematic factors and allocation decisions have provided negative active returns and value added, while the environmental mandates have contributed positively. Table 52 in Appendix F shows summary statistics for gross active returns.

\begin{tabular}{ccc|cc|cc|cc}
\hline & \multicolumn{2}{l|}{ Fund allocation } & \multicolumn{2}{c|}{ Factors } & \multicolumn{2}{c|}{ Environment } & \multicolumn{2}{c}{ Allocation } \\
\cline { 2 - 9 } & Return & VA & Return & VA & Return & VA & Return & VA \\
\hline Gross & -0.05 & -0.15 & -0.04 & -0.22 & 3.85 & 0.19 & -0.05 & -0.14 \\
\hline
\end{tabular}

Notes: The table shows the mean annualized active returns and monthly value added. Returns expressed in percent. Value added is in NOK billion. Sample period: 2013-2021.

Table 18: Equity-fund-allocation realized active return 
Table 19 shows that average active returns and Jensen's alpha have high associated $p$-values. Table

\begin{tabular}{|c|c|c|c|c|c|c|c|c|}
\hline & \multicolumn{2}{|c|}{ Fund allocation } & \multicolumn{2}{|c|}{ Factors } & \multicolumn{2}{|c|}{ Environment } & \multicolumn{2}{|c|}{ Allocation } \\
\hline & Mean & $\begin{array}{c}\text { Beta } \\
\text { adjustment }\end{array}$ & Mean & $\begin{array}{c}\text { Beta } \\
\text { adjustment }\end{array}$ & Mean & $\begin{array}{c}\text { Beta } \\
\text { adjustment }\end{array}$ & Mean & $\begin{array}{c}\text { Beta } \\
\text { adjustment }\end{array}$ \\
\hline $\bar{\alpha}$ & $\begin{array}{r}-0.05 \\
(0.10)\end{array}$ & $\begin{array}{r}-0.12 \\
(0.09)\end{array}$ & $\begin{array}{r}-0.04 \\
(0.07)\end{array}$ & $\begin{array}{r}-0.08 \\
(0.06)\end{array}$ & $\begin{array}{r}3.85^{*} \\
(2.14)\end{array}$ & $\begin{array}{c}3.74 \\
(2.56)\end{array}$ & $\begin{array}{r}-0.05 \\
(0.08)\end{array}$ & $\begin{array}{r}-0.07 \\
(0.08)\end{array}$ \\
\hline$p$-value & .66 & .20 & .59 & .21 & .08 & .15 & .55 & .36 \\
\hline$\beta$ & & $\begin{array}{c}1.01 \\
(0.00)\end{array}$ & & $\begin{array}{l}1.00^{* * *} \\
(0.00)\end{array}$ & & $\begin{array}{c}1.01 \\
(0.06)\end{array}$ & & $\begin{array}{l}1.00^{*} \\
(0.00)\end{array}$ \\
\hline$p$-value & & .00 & & .00 & & .86 & & .08 \\
\hline $\begin{array}{l}N \\
\text { Adjusted } R^{2}\end{array}$ & 105 & $\begin{array}{l}105 \\
1.00\end{array}$ & 105 & $\begin{array}{l}105 \\
1.00\end{array}$ & 105 & $\begin{array}{l}105 \\
0.87\end{array}$ & 105 & $\begin{array}{l}105 \\
1.00\end{array}$ \\
\hline$A R$ & & -0.51 & & -0.58 & & 0.69 & & -0.37 \\
\hline
\end{tabular}

Notes: The table shows the estimated annualized $\alpha$ and $\beta$ from Eq. (1). Returns expressed in percent. Newey and West (1987) standard errors in parentheses. $N$ refers to the number of observations in the period, $R^{2}$ is the explanatory power from the regression, and $A R$ is the appraisal ratio. We denote by stars if $\alpha$ is significantly different from zero and if $\beta$ is significantly different from one. ${ }^{* * * / * * / *}$ indicates significance at the $1 \% / 5 \% / 10 \%$ level.

Table 19: Equity-fund-allocation mean active returns with and without beta adjusting

53 in Appendix F shows that the factor-adjusted alphas are still insignificant for all three strategies and that .76 of the variation of the active return from the systematic-factors strategy can be explained by the five-factor model, while the explanatory power is very low for the other two substrategies $(.15$ and .03).

The results for the factor strategy indicate that NBIM has had small but significant long-term exposures to $M K T, S M B, H M L$, and $R M W$. Table 20 shows that these bets have not yielded a positive active return. The environmental strategy has had a large negative exposure to $H M L$ and $R M W$. The first exposure is in line with the green factor having an indirect exposure to growth (see Pastor, Stambaugh and Taylor, 2021a). However, the returns of the factor exposures go in opposite directions such that, in total, we do not find a large effect on active returns. Finally, the factor model does not explain much of the active return of the fund-allocation strategy.

\begin{tabular}{lrrr|rr|rr|rr}
\hline & & \multicolumn{2}{c|}{ Fund allocation } & \multicolumn{2}{c|}{ Factors } & \multicolumn{2}{c|}{ Environment } & \multicolumn{2}{c}{ Allocation } \\
\cline { 2 - 11 } Factor & Mean & \multicolumn{1}{c}{ Est } & Prod & \multicolumn{1}{|c|}{ Est } & Prod & Est & Prod & Est & Prod \\
\hline$M K T$ & 11.65 & 0.00 & 0.05 & 0.00 & 0.03 & 0.07 & 0.78 & 0.00 & 0.01 \\
$S M B$ & -0.62 & 0.01 & -0.01 & 0.01 & -0.01 & 0.00 & 0.00 & 0.00 & 0.00 \\
$H M L$ & -4.62 & 0.01 & -0.05 & 0.01 & -0.06 & -0.35 & 1.62 & 0.00 & 0.00 \\
$R M W$ & 3.39 & 0.00 & -0.01 & 0.00 & 0.02 & -0.59 & -1.99 & 0.00 & 0.00 \\
$C M A$ & -2.39 & -0.01 & 0.03 & 0.00 & 0.00 & 0.16 & -0.38 & -0.01 & 0.03 \\
\hline Factor sum & & & 0.02 & & -0.02 & & 0.02 & & 0.03 \\
Gross $\alpha$ & & & -0.06 & & -0.02 & & 3.83 & & -0.08 \\
Gross mean & & & -0.05 & & -0.04 & & 3.85 & & -0.05 \\
\hline
\end{tabular}

Notes: The table shows the factor exposures. The Est column shows the beta estimates, and the Mean column shows the average return of the factors. The Prod column shows how much the Fund has gained or lost from the factor exposure, which is the product of the first two columns. Returns expressed in percent.

Table 20: Equity-fund-allocation factor-exposure analysis 


\subsection{Summing up}

For the period under evaluation here, we find that the value added from active management in equity strategies has about three equal sources of positive value added: internal security selection, external security selection, and enhanced indexing. The fund-allocation strategy has destroyed value. Overall, some of the equity strategies seem to have significantly positive active returns, while others do not. 


\section{Fixed-income value creation}

In this section, we first examine the overall fixed-income portfolio before we dig deeper into substrategies in Section 7. Similar to the equity portfolio, the funding of the real-estate portfolio adds complexity to the fixed-income benchmark. NBIM's internal fixed-income benchmark has, since 2017, been adjusted for real-estate funding. This is also the benchmark we examine in this section.

\subsection{Realized value creation}

Figure 19 plots the annualized value added from active management of the fixed-income portfolio. As also shown in Figure 11, value added has been negative several of the last 15 years. A large negative value added in 2008 was followed by an equally large positive active return in 2009. This shows that these strategies, in sum, had exposure to crisis-related events.

We see that the variation of value added increases over time and spikes during the recent financial crises. NBIM (2021c, p. 120) explains fixed-income active returns across years. The accumulated gross value added from 1998 is NOK 36.3 billion. The accumulated costs are NOK 11.8 billion, so the accumulated net value added is NOK 24.5 billion. From 2017 onward, the accumulated gross value added is NOK 31.4 billion, costs are NOK 3.6 billion, and net value added is NOK 27.8 billion. As expected, costs play a much smaller role here than for the equity portfolio.

Figure 20 illustrates the development of the gross and net active returns for the fixed-income portfolio. If we had invested NOK 1,000 in the fixed-income portfolio at inception (1998), it would have yielded NOK 1,040 before and NOK 1,029 after costs in September 2021 compared to investing in the benchmark. Again, we see a large decline during the financial crisis and recovery immediately thereafter. Since then, NBIM has been more restrictive in its fixed-income risk taking. NBIM (2021c,

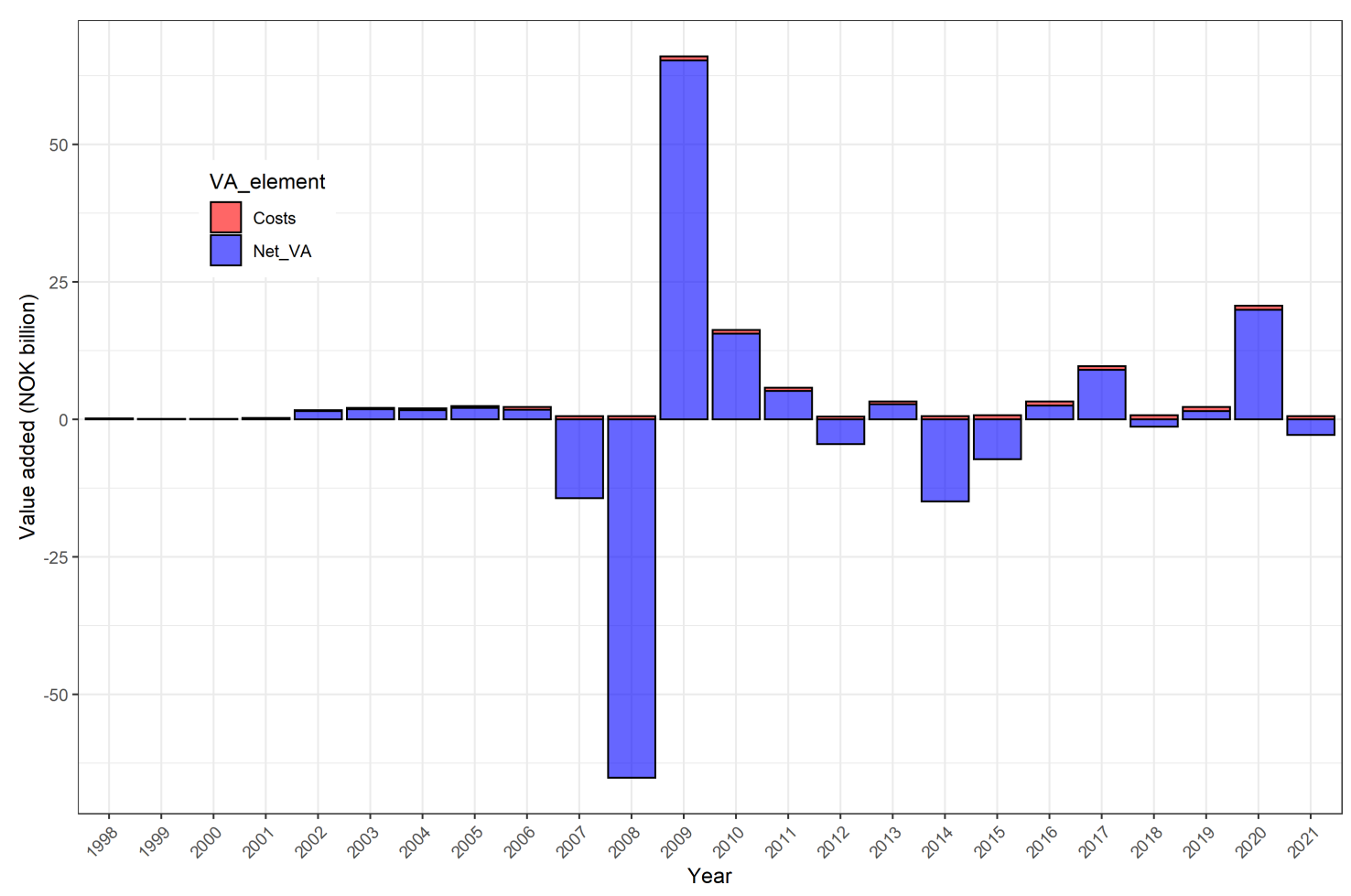

Figure 19: Fixed-income annual value added before and after management costs 


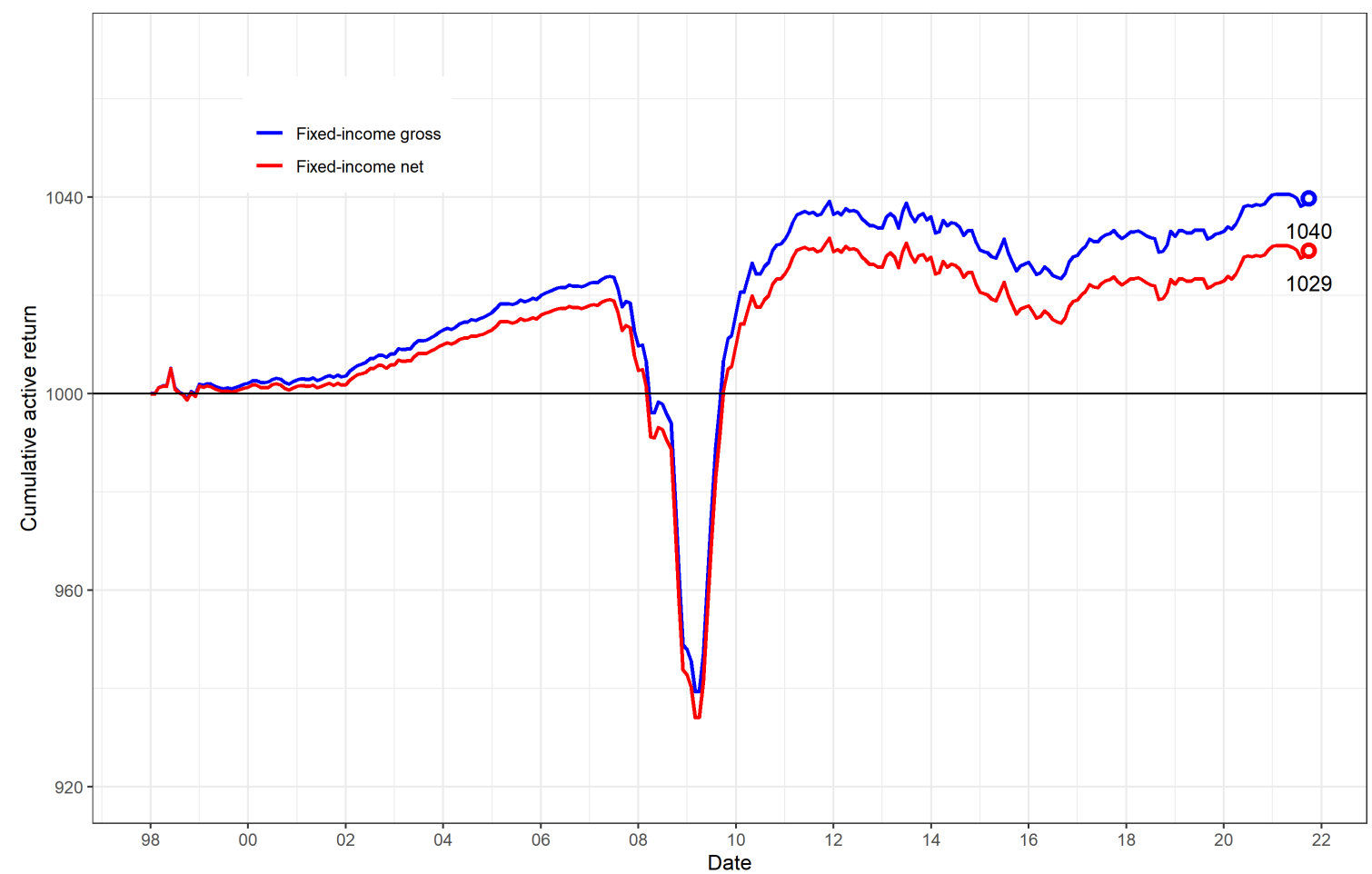

Figure 20: Fixed-income cumulative gross and net active returns

Chart 34) presents a further description of this. Interestingly, the large market downturn in total return during the COVID-crisis in March 2020 did not influence NBIM's active fixed-income returns.

Table 21 presents the annualized average active return and the average monthly value added before and after costs for the fixed-income portfolio. We find that the annualized mean active return is $0.17 \%$ before costs and $0.13 \%$ after costs. The mean monthly value added is NOK 0.13 billions before costs and NOK 0.09 after costs. For the latest sample, the means are larger. For example, the annualized mean active return is $0.24 \%$ before costs.

\begin{tabular}{lcc|cc}
\hline & \multicolumn{2}{c|}{ Full sample (1998-Sep 2021) } & \multicolumn{2}{c}{ Latest sample (2017-Sep 2021) } \\
\cline { 2 - 5 } & Return & VA & Return & VA \\
\hline Gross & 0.17 & 0.13 & 0.24 & 0.55 \\
Costs & 0.04 & 0.04 & 0.03 & 0.06 \\
Net & 0.13 & 0.09 & 0.21 & 0.49 \\
\hline
\end{tabular}

Notes: The table shows the costs and the gross and net mean annualized active returns and monthly value added. Returns expressed in percent. Value added is in NOK billion.

Table 21: Fixed-income realized active returns

\subsection{Evaluation of active returns}

One of the prerequisites of a statistical analysis is a time-consistent process. For the fixed-income return series, we are less certain this holds. "A variety of strategies have been used for the different segments of the fixed-income portfolio in different time periods, but these strategies have overlapped in focus and objective. So while management has at times been dominated by one or more of these strategies, there will often also be elements of the other strategies impacting on the relative return" 
(NBIM, 2021c, p. 71). Thus, we should be careful assuming no regime shifts and also, when it comes to the different fixed-income strategies, we should be aware of the potential changes in and overlap between strategies and substrategies, respectively.

The summary statistics of the gross and net active returns are tabulated in Table 54 in Appendix G. The autocorrelation is high for the longest sample period, while it is much lower for the recent sample period. From Figure 29 in Appendix G, the active returns are almost uncorrelated to benchmark returns, except for extremely negative benchmark returns, where the active returns are also very low. Figure 30 in Appendix $\mathrm{G}$ shows that the distribution of the active returns has less mass on the positive side compared to the equity portfolio in Figure 28.

Figure 21 plots the mean active return and tracking error for the fixed-income portfolio. Since the active return is positive, the $I R$ is also positive. Note that $I R$ for the latest period is much higher than for the total period. The reason is a combination of a higher alpha and a lower tracking error.

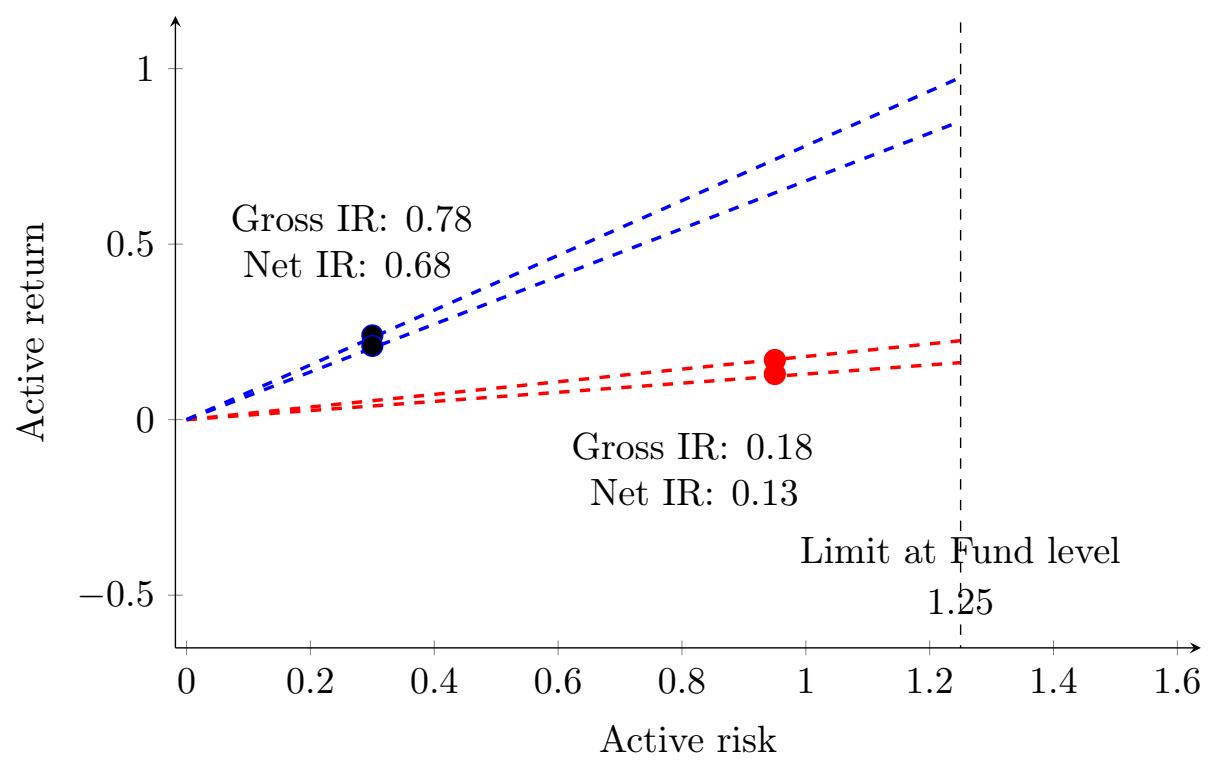

Realized active risk vs ex ante limit. Red: Sample Jan 1998-Sep 2021, Blue: Sample Jan 2017-Sep 2021.

Figure 21: Fixed-income active return-risk space

In Table 22, we evaluate the mean active returns before and after cost for the total and most recent sample periods. For all cases, we find that the means are positive, but all $p$-values are very large (above .13). This implies that the positive active returns for the fixed-income portfolio are not statistically significant.

\begin{tabular}{lcc|cc}
\hline & \multicolumn{2}{c|}{ Full sample (1998-Sep 2021) } & \multicolumn{2}{c}{ Latest sample (2017-Sep 2021) } \\
\cline { 2 - 5 } & Gross & Net & Gross & Net \\
\hline Mean & 0.17 & 0.13 & 0.24 & 0.21 \\
& $(0.45)$ & $(0.45)$ & $(0.15)$ & $(0.15)$ \\
$p$-value & .71 & .78 & .13 & .18 \\
$N$ & 285 & 285 & 57 & 57 \\
\hline
\end{tabular}

Notes: The table shows the estimated annualized mean active returns in percent. Newey and West (1987) standard errors in parentheses. $N$ refers to the number of observations in the period. We denote by stars if the mean is significantly different from zero. ${ }^{* * * / * * / *}$ indicates significance at the $1 \% / 5 \% / 10 \%$ level.

Table 22: Fixed-income mean active returns 


\subsection{Benchmark risk-adjusted performance}

Table 23 presents the beta-adjusted active returns (Jensen's alpha) before and after costs based on estimating Eq. (1) for the fixed-income portfolio. Since the estimated betas for the full sample period are above one, and the excess returns on the portfolio and the benchmark are positive, the risk-adjusted active returns are lower than without the beta adjustment. Still, notice that the estimated betas are not significantly different from one. For the whole sample, the gross and net alphas are $0.09 \%$ and $0.05 \%$, respectively. The estimated alphas are larger in the most recent sample period. All alpha estimates have very large associated $p$-values. Hence, we can conclude that the beta-adjusted performance of the fixed-income portfolio is not significantly positive.

\begin{tabular}{lcc|cc}
\hline & \multicolumn{2}{l|}{ Full sample (1998-Sep 2021) } & \multicolumn{2}{c}{ Latest sample (2017-Sep 2021) } \\
& Gross & Net & Gross & Net \\
\hline$\alpha$ & 0.09 & 0.05 & 0.24 & 0.21 \\
& $(0.42)$ & $(0.42)$ & $(0.16)$ & $(0.16)$ \\
$p$-value & .83 & .91 & .14 & .19 \\
$\beta$ & 1.03 & 1.03 & 1.00 & 1.00 \\
& $(0.02)$ & $(0.02)$ & $(0.01)$ & $(0.01)$ \\
$p$-value & .14 & .14 & .84 & .84 \\
$N$ & 285 & 285 & 57 & 57 \\
$R^{2}$ & .98 & .98 & 1.00 & 1.00 \\
$A R$ & 0.10 & 0.05 & 0.78 & 0.69 \\
\hline
\end{tabular}

Notes: The table shows the estimated annualized $\alpha$ and $\beta$ from Eq. (1) using gross and net returns in percent. Newey and West (1987) standard errors in parentheses. $R^{2}$ is the explanatory power from the regression, and $A R$ is the appraisal ratio. We denote by stars if $\alpha$ is significantly different from zero and if $\beta$ is significantly different from one. ${ }^{* * * / * * / *}$ indicates significance at the $1 \% / 5 \% / 10 \%$ level.

Table 23: Fixed-income mean beta-adjusted active returns

For the total sample, the $R^{2}$ is .98 , while for the recent period, it is 1.00. Again, this result confirms that the variation in the benchmark explains almost all the variation of the Fund's fixed-income return, meaning a low degree of active management relative to the internal fixed-income benchmark. The $A R$ is positive for all four alternatives, but very low for the full sample period.

\subsection{Factor risk-adjusted performance}

Regarding fixed-income-portfolio performance attribution gathers little consensus on the choice of the appropriate factor model. As described in Cremers et al. (2019): "No model is generally accepted for controlling for bond portfolio risks. As a result, a wide variety of models have been used." Two of the most applied factors in academic research are the term-premium and default-premium factors. This model is also consistent with the advice from Dahlquist et al. (2015, p. 14, Suggestion 2).28

To analyze the Fund's fixed-income portfolio, we consider a restricted version of the seven-factor model in Eq. (2) and use the following two-factor model:

$$
R_{t}^{\mathrm{a}}=\alpha+\beta_{T E R M} T E R M_{t}+\beta_{D E F} D E F_{t}+\epsilon_{t} .
$$

\footnotetext{
${ }^{28}$ For further guidance on richer fixed-income factor models, see, for example, Bai, Bali and Wen (2019), Natter, Rohleder and Wilkens (2021), and Brooks, Palhares and Richardson (2018)
} 
Table 24 presents the estimated factor model for the fixed-income portfolio for the entire and the latest sample periods. We find that the estimated alphas are still positive for all portfolios. The annualized alphas before and after cost for the entire period are $0.12 \%$ and $0.08 \%$, respectively. Furthermore, we find that the $p$-values are very high. For the most recent period, the alpha estimates are higher at $0.27 \%$ and $0.24 \%$, respectively. These alpha estimates are also higher than the corresponding means from Table 22 and the benchmark adjusted alphas from Table 23. For the most recent period, the associated $p$-values are closer to regular levels of statistical significance.

\begin{tabular}{|c|c|c|c|c|}
\hline & \multicolumn{2}{|c|}{ Full sample (1998-Sep 2021) } & \multicolumn{2}{|c|}{ Latest sample (2017-Sep 2021) } \\
\hline & Gross & Net & Gross & Net \\
\hline $\bar{\alpha}$ & 0.12 & 0.08 & $0.27^{*}$ & $0.24^{*}$ \\
\hline & $(0.31)$ & $(0.31)$ & $(0.14)$ & $(0.14)$ \\
\hline$p$-value & .70 & .81 & .07 & .10 \\
\hline TERM & $\begin{array}{c}-0.022^{* *} \\
(0.01)\end{array}$ & $\begin{array}{c}-0.02^{* *} \\
(0.01)\end{array}$ & $\begin{array}{c}-0.011^{* *} \\
(0.01)\end{array}$ & $\begin{array}{c}-0.01^{* *} \\
(0.01)\end{array}$ \\
\hline$p$-value & .03 & .03 & .02 & .02 \\
\hline$D E F$ & $\begin{array}{c}0.06^{*} \\
(0.03)\end{array}$ & $\begin{array}{c}0.06^{*} \\
(0.03)\end{array}$ & $\begin{array}{c}0.002 \\
(0.003)\end{array}$ & $\begin{array}{c}0.002 \\
(0.003)\end{array}$ \\
\hline$p$-value & .06 & .06 & .57 & .57 \\
\hline$N$ & 285 & 285 & 57 & 57 \\
\hline Adjusted $R^{2}$ & 0.21 & 0.21 & 0.04 & 0.04 \\
\hline
\end{tabular}

Notes: The table shows the estimated coefficients from the factor model in Eq. (4). $\alpha$ is annualized. Returns expressed in percent. Newey and West (1987) standard errors in parentheses. $N$ refers to the number of observations in the period, and $R^{2}$ is the explanatory power from the regression. We denote by stars if $\alpha$ is significantly different from zero and if $\beta$ is significantly different from one. ${ }^{* * * / * * / *}$ indicates significance at the $1 \% / 5 \% / 10 \%$ level.

Table 24: Fixed-income factor model risk adjustment

The explanatory power of the two-factor model is relative low. The adjusted $R^{2}$ is .21 for the full sample and .04 for the recent sample period.

Table 25 quantifies how the factor exposures influence the factor-adjusted alphas. The Fund has gained $0.05 \%$ from its fixed-income portfolio's factor exposure, while it has lost $0.03 \%$ in the most recent sample period.

\begin{tabular}{|c|c|c|c|c|c|c|}
\hline \multirow[b]{2}{*}{ Factor } & \multicolumn{3}{|c|}{ Full sample (1998-Sep 2021) } & \multicolumn{3}{|c|}{ Latest sample (2017-Sep 2021) } \\
\hline & Est & Mean & Prod & Est & Mean & Prod \\
\hline TERM & -0.02 & 3.43 & -0.05 & -0.01 & 2.94 & -0.04 \\
\hline$D E F$ & 0.06 & 1.76 & 0.10 & 0.00 & 4.38 & 0.01 \\
\hline Factor sum & & & 0.05 & & & -0.03 \\
\hline Net $\alpha$ & & & 0.08 & & & 0.24 \\
\hline Costs & & & 0.04 & & & 0.03 \\
\hline Gross mean & & & 0.17 & & & 0.24 \\
\hline
\end{tabular}

Notes: The table shows the factor exposures. In the Est column we show the beta estimates and in the Mean column we show the average return of the factors. In the Prod column, we show how much the Fund has gained or lost from the factor exposure, which is the product of the first two columns. Returns expressed in percent.

Table 25: Fixed-income factor-exposure analysis 


\subsection{Summing up}

Since 1998, the Fund has earned NOK 25 billion after costs at the total fixed-income level. The average annualized active return is $0.17 \%$ before costs and $0.13 \%$ after costs. Also, since 2017, these numbers have been positive. Overall, the various measures of the active returns of the Fund's fixed-income portfolios are not significantly different from zero. 


\section{$7 \quad$ Fixed-income strategies}

It is harder to get an overview of the fixed-income strategies than the equity strategies. NBIM (2021c, Section 2) describes the history of the fixed-income strategy. Since NBIM has terminated some of the prevalent strategies around the financial crisis in 2008, we cannot map these into the Fund's current structure. Figure 22 shows the current substrategies for which we have data. Notice that the structure of the substrategies is similar to equity substrategies. However, fixed-income strategies include neither external security selection nor systematic factors.

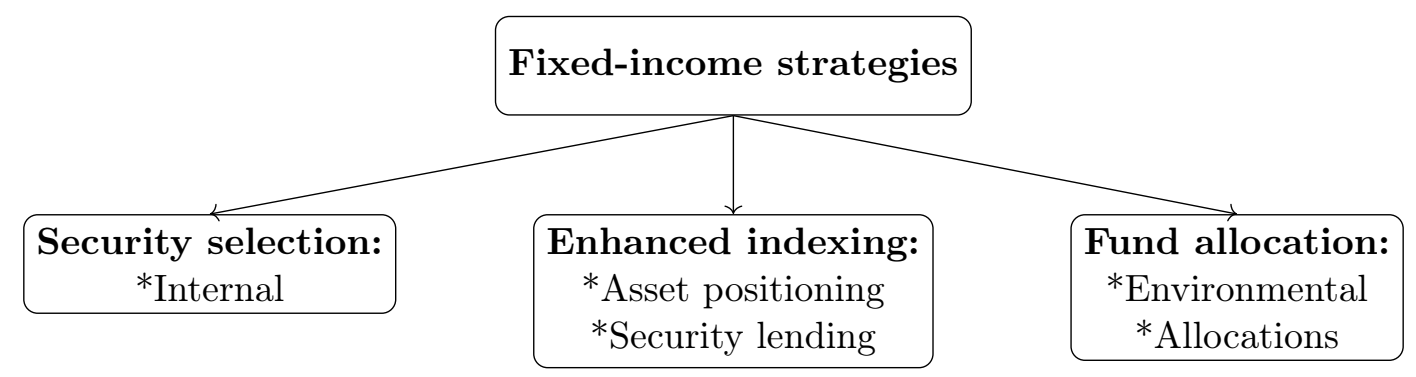

Figure 22: Fixed-income substrategies

Given data availability, we examine monthly returns for the different strategies since the start of 2013. Again, this is a period with fairly steady markets following the turmoil caused by the global financial crisis. Also, note that not all strategies started in $2013 .{ }^{29}$ Table 55 in Appendix H shows the means and standard deviations for the fixed-income subportfolios and the corresponding internal benchmarks. Figure 31 in Appendix H presents the different strategies' AUMs.

\begin{tabular}{|rrrrr} 
& & \multicolumn{3}{c}{ Allocation } \\
& Environment & -.01 \\
& & & \\
Lending & -.01 & -.04 \\
\hline & & & \\
\hline Positioning & -.04 & .04 & .27 \\
\hline Internal & -.46 & -.01 & -.22 & -.37
\end{tabular}

Unconditional active-return correlation matrix, green background if negative correlation, and red if positive (in contrast to the returns reported in Section 9). Sample: 2013-2021.

Figure 23: Fixed-income strategies correlation matrix

Figure 23 shows the correlation matrix for the fixed-income substrategies. ${ }^{30}$ We find that the internal-security-selection strategy has a negative correlation with the other strategies. For example, the correlation coefficient is -.46 with the asset positioning strategy. Not surprisingly, security lending is almost uncorrelated with all other substrategies.

\footnotetext{
${ }^{29}$ The fixed-income internal-security-selection strategy began in October 2014 and the fixed-income security-lending began in November 2015. For the fixed-income environmental strategy, we only have data for January 2016 to June 2020.

${ }^{30}$ Each correlation is calculated using the longest overlapping sample period for the two strategies.
} 


\subsection{Security selection}

The security-selection strategy started in October 2014. NBIM (2021c, p. 99) describes the current strategy.

\section{Results}

The accumulated gross value added related to internal security selection is NOK 8 billion. The accumulated costs are NOK 2 billion. Hence, the accumulated net value added amounts to NOK 6 billion. Table 26 presents the mean gross and net active return and value added. We find that the net annualized mean active return for this strategy is $0.36 \%$. Table 56 in Appendix $H$ shows the summary statistics for the gross and net returns. Notice that the success ratio for positive active returns is below .50 .

\begin{tabular}{lcc}
\hline & \multicolumn{2}{c}{ Internal security selection } \\
\cline { 2 - 3 } & Return & VA \\
\hline Gross & 0.41 & 0.09 \\
Costs & 0.06 & 0.02 \\
Net & 0.36 & 0.07 \\
\hline
\end{tabular}

Notes: The table shows the costs and the gross and net mean annualized active returns and monthly value added. Returns expressed in percent. Value added is in NOK billion. Sample period: 2014-2021.

Table 26: Fixed-income-security-selection realized active returns

Table 27 shows the average active returns before and after costs and the beta-adjusted alpha before and after costs. The overall message is that even if the active returns and alphas are positive, they are not significant at conventional significance levels ( $p$-values between .10 and .24). It is also worth noting that the beta estimates are below one.

\begin{tabular}{lcc|cc}
\hline & \multicolumn{2}{c|}{ Security selection before } & \multicolumn{2}{c}{ Security selection after } \\
\cline { 2 - 5 } & Mean & Beta adjustment & Mean & Beta adjustment \\
\hline$\alpha$ & 0.41 & $0.59^{*}$ & 0.36 & 0.54 \\
& $(0.31)$ & $(0.36)$ & $(0.31)$ & $(0.36)$ \\
$p$-value & .18 & .10 & .24 & .14 \\
$\beta$ & & $0.93^{* * *}$ & & $0.93^{* * *}$ \\
& & $(0.02)$ & & $(0.02)$ \\
$p$-value & & .00 & & .00 \\
$N$ & 84 & 84 & 84 & 84 \\
Adjusted $R^{2}$ & & 0.98 & & 0.98 \\
$A R$ & & 0.75 & & 0.69 \\
\hline
\end{tabular}

Notes: The table shows the mean annualized active return in the Mean column and the estimated annualized $\alpha$ and $\beta$ from Eq. (1) in the Beta adjustment column. Returns expressed in percent. Newey and West (1987) standard errors in parentheses. $N$ refers to the number of observations in the period, $R^{2}$ is the explanatory power from the regression, and $A R$ is the appraisal ratio. We denote by stars if $\alpha$ is significantly different from zero and if $\beta$ is significantly different from one. ${ }^{* * * / * * / *}$ indicates significance at the $1 \% / 5 \% / 10 \%$ level.

Table 27: Fixed-income-security-selection mean active returns with and without beta adjusting 
Table 28 shows the factor analysis results. The internal-security-selection strategy has a negative exposure to the $D E F$ factor. As shown in Table 57 in Appendix $H$, this is a significant bet and has resulted in a loss so far. The alpha adjusted for factor risk is significantly positive, with a $p$-value of .04 .

\begin{tabular}{|c|c|c|c|}
\hline \multirow[b]{2}{*}{ Factor } & \multicolumn{3}{|c|}{ Internal security selection } \\
\hline & Mean & Est & Prod \\
\hline TERM & 3.56 & 0.01 & 0.04 \\
\hline$D E F$ & 3.49 & -0.07 & -0.24 \\
\hline Sum factor & & & -0.19 \\
\hline Net $\alpha$ & & & 0.55 \\
\hline Cost & & & 0.05 \\
\hline Gross mean & & & 0.41 \\
\hline
\end{tabular}

Notes: The table shows the factor exposures. The Est column shows the beta estimates, and the Mean column shows the average return of the factors. The Prod column shows how much the Fund has gained or lost from the factor exposure, which is the product of the first two columns. Returns in percent.

Table 28: Fixed-income-security-selection factor-exposure analysis

\subsection{Enhanced indexing}

In line with the equity strategies, fixed income includes two enhanced-indexing strategies: asset positioning and security lending. In NBIM's words: "The asset positioning strategy is used for managing government, government related, and covered bonds. The objective for the strategy is to cost-efficiently implement the desired market exposure, and to generate excess return over time. This is done through a variety of relative value and tactical macro strategies, tailored to the Fund's unique characteristics. The investment universe is divided up, and the capital allocated to each segment are given to specialist teams that dynamically exploit opportunities within their markets. Many of the strategies that are pursued within these investment mandates take advantage of anomalies that stem from market inefficiencies. Typical examples are new issue premium harvesting, and relative value strategies across instruments, sectors, and issuers. In addition, tactical positions initiated can be more large scale. These usually stems from a perception that risk premiums embedded in the fixed-income universe are out of line with fundamentals, or they are related to transition activity, and hence motivated by reduction in transaction cost" (NBIM, 2021c). While we have data for the entire period for asset positioning, we only have data for security lending after November 2015.

\section{Results}

The accumulated gross value added from 2013 and onward is NOK 46 billion in total, NOK 40 billion from asset positioning and NOK 4 billion from security lending. Table 29 shows that the assetpositioning strategy has an average annualized active return of $0.25 \%$ and an average value added of NOK 0.38 billion per month. The numbers for the security-lending strategy are much lower. Even if the active returns are lower than for security selection, it is earned on a much larger asset base. Table 58 in Appendix H shows the summary statistics for the gross returns. Notice the high hit ratios. 


\begin{tabular}{ccc|cc|cc}
\hline & \multicolumn{2}{c|}{ Enhanced } & \multicolumn{2}{c|}{ Positioning } & \multicolumn{2}{c}{ Security lending } \\
\cline { 2 - 7 } Return & VA & Return & VA & Return & VA \\
Gross & 0.28 & 0.43 & 0.25 & 0.38 & 0.03 & 0.05 \\
\hline
\end{tabular}

Notes: The table shows the gross mean annualized active returns and monthly value added. Returns expressed in percent. Value added is in NOK billion. Sample period: 2013-2021, except security lending (Nov 2015-2021).

Table 29: Fixed-income-enhanced-indexing realized active returns

Table 30 documents that the enhanced-indexing strategies have very low standard deviations, leading to significantly positive active returns and alphas ( $p$-values below .01). From the factor analysis, documented in Table 59 and 60 in Appendix H, we find that the estimated factor-adjusted alphas are very similar to the average active returns, which implies the factors barely affect them. The adjusted $R^{2}$ s of the factor models are low.

\begin{tabular}{lcc|cc|cc}
\hline & \multicolumn{2}{c|}{ Enhanced } & \multicolumn{2}{c|}{ Positioning } & \multicolumn{2}{c}{ Lending } \\
\cline { 2 - 7 } & Mean & Beta adjust. & Mean & Beta adjust. & Mean & Beta adjust. \\
\hline$\alpha$ & $0.28^{* * *}$ & $0.28^{* * *}$ & $0.25^{* * *}$ & $0.24^{* * *}$ & $0.03^{* * *}$ & $0.03^{* * *}$ \\
$p$-value & $(0.05)$ & $(0.05)$ & $(0.05)$ & $(0.05)$ & $(0.01)$ & $(0.01)$ \\
$\beta$ & .0000 & .00 & .0000 & .0000 & .00 & .00 \\
& & 1.00 & & 1.00 & & 1.00 \\
$p$-value & & $(0.00)$ & & $(0.00)$ & & $(0.00)$ \\
$N$ & .29 & & .30 & & .70 \\
Adjusted $R^{2}$ & 105 & 105 & 105 & 105 & 71 & 71 \\
AR & 0.00 & 1.00 & 0.00 & 1.00 & 0.00 & 1.00 \\
\hline
\end{tabular}

Notes: The table shows the mean active return in the Mean column and the estimated $\alpha$ and $\beta$ from Eq. (1) in the Beta adjustment column. Returns expressed in percent. Newey and West (1987) standard errors in parentheses. $N$ refers to the number of observations in the period, $R^{2}$ is the explanatory power from the regression, and $A R$ is the appraisal ratio. We denote by stars if $\alpha$ is significantly different from zero and if $\beta$ is significantly different from one. ${ }^{* * * / * * / *}$ indicates significance at the $1 \% / 5 \% / 10 \%$ level.

Table 30: Fixed-income-enhanced-indexing mean active returns with and without beta adjusting

\subsection{Fund allocation}

NBIM (2021c) gives limited detail on the substrategies in this category. NBIM has given us the following description: "Managing of assets that has an allocation-type risk profile, including environmental portfolios (green bonds). Over allocation and funding of emerging-market fixed income and positions related to fiscal strength are included under fund allocation, in addition to other top level decisions taken in the Reference Portfolio." For the environmental strategy, we have a short sample period, January 2016 to June 2020.

\section{Results}

The accumulated gross value added is NOK -35 billion for the total fund-allocation strategy; almost all of this stems from the allocation strategy. For the environmental mandate, the accumulated value added from 2016 to 2020 is NOK 0.002 billion. Table 31 shows that the annualized average active 


\begin{tabular}{ccc|cc|cc}
\hline & \multicolumn{2}{l|}{ Fund allocation } & \multicolumn{2}{|c|}{ Environment } & \multicolumn{2}{c}{ Allocation } \\
\cline { 2 - 7 } Gross & Return & VA & Return & VA & Return & VA \\
Gro & -0.33 & 0.28 & 0.00 & -0.18 & -0.33 \\
\hline
\end{tabular}

Notes: Means annualized by multiplying the mean monthly return by 1200. Returns expressed in percent. Value added is in NOK billion. Sample period: 2013-2021.

Table 31: Fixed-income-fund-allocation realized active return

\begin{tabular}{lcc|cc|cc}
\hline & \multicolumn{2}{c|}{ Fund allocation } & \multicolumn{2}{c|}{ Environmental } & \multicolumn{2}{c}{ Allocation } \\
\cline { 2 - 7 } & Mean & Beta adjustment & Mean & Beta adjustment & Mean & Beta adjustment \\
\hline$\alpha$ & -0.18 & -0.16 & 0.28 & 0.31 & -0.18 & -0.16 \\
$p$-value & $(0.11)$ & $(0.12)$ & $(0.20)$ & $(0.19)$ & $(0.11)$ & $(0.12)$ \\
$\beta$ & .11 & .21 & .17 & .11 & .11 & .21 \\
& & 0.98 & & 0.99 & & 0.98 \\
$p$-value & & $(0.01)$ & & $(0.01)$ & & $(0.01)$ \\
$N$ & .12 & & .28 & & .12 \\
Adjusted $R^{2}$ & 105 & 105 & 54 & 54 & 105 & 105 \\
AR & & 0.99 & & 0.99 & & 0.99 \\
\hline
\end{tabular}

Notes: The table shows the mean annualized active return in the Mean column and the estimated annualized $\alpha$ and $\beta$ from Eq. (1) in the Beta adjustment column. Returns expressed in percent. Newey and West (1987) standard errors in parentheses. $N$ refers to the number of observations in the period, $R^{2}$ is the explanatory power from the regression, and $A R$ is the appraisal ratio. We denote by stars if $\alpha$ is significantly different from zero and if $\beta$ is significantly different from one. ${ }^{* * * / * * / *}$ indicates significance at the $1 \% / 5 \% / 10 \%$ level.

Table 32: Fixed-income-fund-allocation mean active returns with and without beta adjusting

return is $0.28 \%$ for the environmental mandate and $-0.18 \%$ for the allocation strategy. These numbers reflect that allocation bets have been challenging for the Fund. As seen in Table 32, even if the active allocation returns are negative, they are not statistically significant ( $p$-values above .11).

The factor analysis in Table 33 shows that $-0.10 \%$ of allocation's $-0.18 \%$ can be attributed to a negative exposure to TERM. Table 62 in Appendix $H$ shows that this is a significant negative exposure. After factor adjusting, the alphas remain insignificant.

\begin{tabular}{lrrr|rr|rr}
\hline & & \multicolumn{2}{c|}{ Fund allocation } & \multicolumn{2}{c|}{ Environment } & \multicolumn{2}{c}{ Allocation } \\
\cline { 3 - 8 } Factor & Mean & Est & Prod & Est & Prod & Est & Prod \\
\hline TERM & 3.58 & -0.03 & -0.10 & 0.00 & 0.00 & -0.03 & -0.10 \\
$D E F$ & 3.58 & 0.01 & 0.04 & 0.01 & 0.03 & 0.01 & 0.04 \\
\hline Factor sum & & & -0.06 & & 0.03 & & -0.06 \\
Gross $\alpha$ & & & -0.12 & & 0.25 & & -0.12 \\
Gross mean & & & -0.18 & & 0.28 & & -0.18 \\
\hline
\end{tabular}

Notes: The table shows the factor exposures. The Est column shows the beta estimates, and the Mean column shows the average return of the factors. The Prod column shows how much the Fund has gained or lost from the factor exposure, which is the product of the first two columns. Returns expressed in percent.

Table 33: Fixed-income-fund-allocation factor-exposure analysis 


\subsection{Summing up}

For active fixed-income strategies, most of the value added comes from enhanced indexing. Internal security selection also has a small positive contribution, while fund allocation has contributed negatively. Only few of the fixed-income substrategies have average active returns significantly different from zero. 


\section{Real-estate value creation}

In this section, we analyze the contribution of the real-estate portfolio to the Fund's risk and return since 2017. ${ }^{31}$ First, in Section 8.1, we introduce the real-estate portfolio, then we consider the performance of the total real-estate portfolio in Section 8.2, followed by the performance of the unlisted (Section 8.3) and listed (Section 8.4) real-estate portfolios. Section 8.5 discusses the financing model and, finally, we sum up in Section 8.6.

\subsection{Real-estate portfolio}

We learn about the history of NBIM's real-estate investments from NBIM (2020d). NBIM has invested in real estate since 2010. In the period 2010-2017, the maximum permissible allocation to real estate was $5 \%$ of the Fund's AUM. The Fund's real-estate investments concentrated on a few large cities in developed countries, such as London, and concentrated in three sectors, office, retail, and logistics. NBIM targeted joint ventures for unlisted-real-estate investments. The Fund invested in unlisted as well as listed real estate. They measured the performance of the Fund's real-estate investments against a broad real-estate benchmark, IPD Global Property Index (exclusive of Norway) (see Dahlquist and $\varnothing$ degaard, 2018). Furthermore, NBIM has indicated to us that, according to their mandate, the benchmark was formally the actual real-estate investments.

The Fund's current (since 2017) real-estate-investment practice (including the choice of benchmark) follows the advice from an external expert group's report on this topic (van Nieuwerburgh, Stanton and de Bever, 2015). The main change is the real-estate benchmarks. Currently, the Fund's benchmarks for its real-estate investments are internally created and consist of equity and fixed-income assets. NBIM uses separate benchmarks for the unlisted and listed-real-estate investments, whereas the total realestate benchmark is the aggregation of the two. We will discuss the choice of real-estate benchmarks further in Section 8.5. Now, the maximum permissible allocation to unlisted real estate is $7 \%$ of the Fund's AUM. NBIM has effectively used a $5 \%$ limit due to buffer needs and tracking-error limits (see NBIM, 2020d). NBIM has continued to focus on real-estate investments similar to those from before 2017, investing in both listed and unlisted real estate.

Since the MoF changed the real-estate benchmarking completely in 2017, it is natural (and in accordance with our mandate) to begin the analysis of the performance of the real-estate portfolio the same year. The real-estate returns before 2017 are included in the Fund's overall portfolio returns, but they are not treated here. For the listed-real-estate portfolio, we thereby have 57 monthly observations. The appraisal of the unlisted-real-estate portfolio occurs every quarter, so we consider quarterly returns for this investment strategy. This implies that we have even fewer observations for the unlisted-realestate portfolio, 19 quarterly observations. The total real-estate portfolio is the sum of the listed- and unlisted-real-estate portfolios, so we use quarterly observations for the total real-estate portfolio.

It is important to be cautious when interpreting the performance evaluation for the Fund's realestate portfolios, first because we only have few time-series observations covering less than five years. This implies that there is a high degree of uncertainty in all statistics. Second, the returns on the

\footnotetext{
${ }^{31}$ Real-estate investments are of current interest to academics as well as industry investors, which is evident from the recent special issue on real and private value assets in the Review of Financial Studies (Goetzmann, Spaenjers and van Nieuwerburgh, 2021).
} 


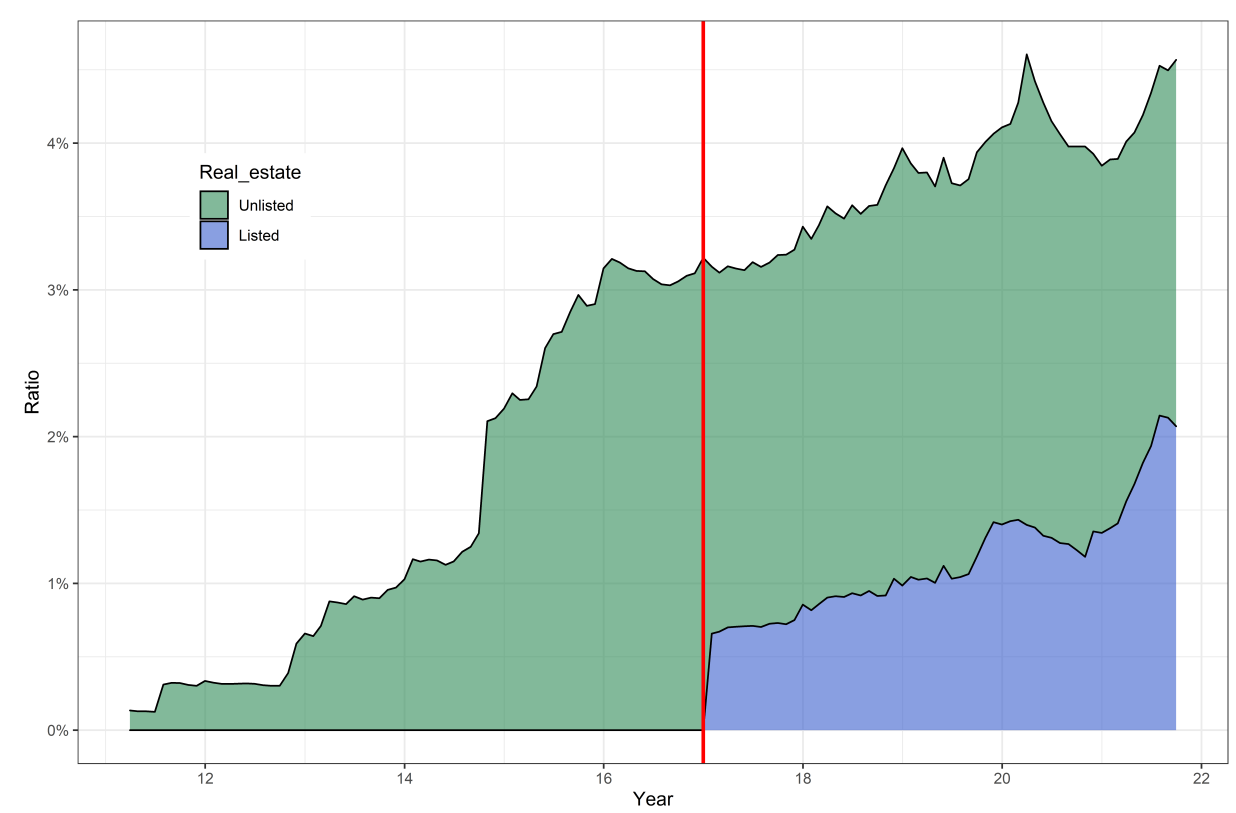

Fraction of the Fund in real estate. Red vertical line in 2017 indicates when the new funding model for real estate was launched and listed real estate was transferred from the equity portfolio to the real-estate portfolio.

Figure 24: Real-estate fraction of Fund AUM

unlisted-real-estate investments are smoothed due to the appraisal process and then by construction the same applies for the returns on the total real-estate investments. Third, the benchmarks given to us are not real-estate benchmarks, so they might not be relevant for performance evaluation. Due to these issues, the reader should put little emphasis on any specific numbers in the performance evaluation below. Therefore, we deemphasize the real-estate portfolios' active returns and value added by tabulating them in Appendix I.

Figure 32 in Appendix I shows the quarterly time series of the AUM in NOK billion for the total, unlisted-, and listed-real-estate portfolios. Figure 24 shows the corresponding development as a percentage of the Fund's AUM. The vertical red line marks the change in the benchmark in 2017. The AUM for the unlisted-real-estate portfolio is increasing steadily until the onset of the COVID crisis in 2020, after which there is a slight decline. The listed-real-estate portfolio has also decreased, but it then increased in 2021. Currently, the listed- and unlisted-real-estate portfolios are similar in size.

\subsection{Total portfolio}

Table 34 shows the descriptive statistics for the gross and net returns of the total real-estate portfolio. The portfolio annualized average gross return is $6.62 \%$ which is much lower than for the Fund's total portfolio during the same sample period $(10.73 \%)$. The standard deviation is also smaller than for the total portfolio ( $7.67 \%$ compared to $11.32 \%$ ). The low standard deviation is most likely caused by the smoothed returns. The Sharpe ratio for the gross return is 0.72 which is smaller than for the total portfolio (0.85). So, the total real-estate portfolio has lower returns and is less risky than the Fund's total portfolio. The average return and standard deviation are between the Fund's equity and fixed-income portfolios, while the Sharpe ratio is smaller than for equity and similar to fixed income. The costs of the total real-estate portfolio are larger than for the other investment strategies (Figure 5 in Section 2.3). 


\begin{tabular}{lcc}
\hline & Gross return Net return \\
\hline Mean (\%) & 6.62 & 6.50 \\
Std.dev. (\%) & 7.67 & 7.66 \\
Sharpe ratio & 0.72 & 0.71 \\
\hline
\end{tabular}

Notes: The table shows the annualized mean and standard deviation of the gross and net returns and Sharpe ratios. Returns expressed in percent. Sample period: 2017-2021.

Table 34: Total-real-estate total return and risk

Table 63 in Appendix I shows that the annualized average gross active return is negative at $-1.50 \%$ ( $p$-value of .64). ${ }^{32}$ The realized tracking error is very large $5.04 \%$. The average gross valued added per quarter of the total real-estate portfolio is NOK -1.63 billion.

We understand from our conversations with NBIM, as well as from NBIM (2020d), that the Fund also holds the real-estate portfolio to obtain diversification benefits compared to only holding equity and fixed income. Diversification benefits are available because the returns on the real-estate portfolio do not strongly correlate with the rest of the Fund's portfolio. ${ }^{33}$ Adding real-estate investments to the Fund's equity and fixed-income portfolios may reduce the Fund portfolio's overall risk by reducing its standard deviation. Due to the limited data available for the Fund's real-estate investments, we do not quantify the diversification benefits. But it should be expected that the diversification benefits are fairly small: The real-estate portfolio represents only about $5 \%$ of the Fund's AUM.

\subsection{Unlisted portfolio}

In this subsection, we describe the performance of the Fund's unlisted-real-estate portfolio. Table 35 shows the summary statistics for the performance of the Fund's unlisted-real-estate portfolio. Its average gross return is higher than for the total real-estate portfolio, but the standard deviation is lower. This results in a Sharpe ratio of 1.53, much higher than for the Fund's total portfolio. The standard deviation in Table 35 suggests low risk, but the low standard deviation probably results from the smoothed returns. The costs are larger than for the total real-estate portfolio.

\begin{tabular}{lcc}
\hline & Gross return Net return \\
\hline Mean (\%) & 7.04 & 6.88 \\
Std. dev. (\%) & 3.90 & 3.89 \\
Sharpe ratio & 1.53 & 1.49 \\
\hline
\end{tabular}

Notes: The table shows the mean and standard deviation of the annualized gross and net returns and the Sharpe ratio. Returns expressed in percent. Sample period: 2017-2021.

Table 35: Unlisted-real-estate total return and risk

Table 64 in Appendix I shows that the annualized gross and net mean active return is $-0.13 \%$ ( $p$-value of .96). The average quarterly gross value added is NOK -0.32 billion. The annualized realized

\footnotetext{
${ }^{32}$ The high average return on the benchmark relative to the real-estate portfolio could be caused by favorable market conditions for equity and fixed income during the sample period combined with unfavorable real-estate market conditions.

${ }^{33}$ The correlation coefficient for the quarterly returns of the total real-estate portfolio with the equity portfolio is .77 and .40 with fixed income.
} 
tracking error of the unlisted-real-estate portfolio is $6.22 \%$, even larger than for the total real-estate portfolio.

\subsection{Listed portfolio}

In this subsection, we describe the performance of the Fund's listed-real-estate portfolio. Table 36 shows the summary statistics for the listed-real-estate portfolio's performance. ${ }^{34}$ The average gross return is smaller for the unlisted- than for the listed-real-estate portfolio, but the standard deviation is much larger. This results in a low Sharpe ratio of 0.29 relative to the listed-real-estate portfolio, as well as to the Fund's total portfolio.

\begin{tabular}{lc}
\hline & Gross return \\
\hline Mean (\%) & 6.53 \\
Std. dev. (\%) & 18.66 \\
Sharpe ratio & 0.29 \\
\hline Notes: The table shows the mean \\
and standard deviation of the annu- \\
alized gross return and the Sharpe \\
ratio. Returns expressed in percent. \\
Sample period: 2017-2021.
\end{tabular}

Table 36: Listed-real-estate total return and risk

Table 65 in Appendix I shows that the annualized average gross active return is very large and negative at $-3.81 \%$ ( $p$-value of .45). The gross average monthly value added is NOK -0.46 billion. The annualized realized tracking error for the listed-real-estate portfolio is very large, $12.29 \%$. Even if there might be differences between the realized and NBIM's ex-ante tracking error, the numbers are large and "eat" a lot of the total tracking-error budget. Figure 33 in Appendix I presents a scatter plot of the monthly active returns against the benchmark returns. Notice the very low benchmark and active return in March 2020, the beginning of the COVID crisis. The active returns tend to be low when the benchmark is low. The listed-real-estate portfolio's active returns are highly volatile, mainly because the portfolio returns are highly volatile, much more so than those of the benchmark.

\subsection{Financing model for real-estate portfolio}

The Fund's financing model for its real-estate investments implies that the Fund buys real estate in a given country (say, the United Kingdom) by selling equity and fixed income from the same country issued in the same currency (British pounds), typically in the proportions of around $70 \%$ fixed income and 30\% equity (see NBIM, 2020d). Then, NBIM constructs its real-estate benchmarks to resemble the Fund's financing model.

The benchmarks are constructed from the Fund's overall benchmark given by the MoF by adjusting the weights in equity and fixed income. ${ }^{35}$ For the unlisted real estate, the weight in the benchmark fits the currency composition of the Fund's actual real-estate investments, while for the listed-real-estate

\footnotetext{
${ }^{34}$ We do not have the costs for the listed-real-estate portfolio. We understand from our conversations with NBIM that the costs are small relative to those of the unlisted-real-estate portfolio.

${ }^{35}$ The description of the real-estate benchmarks stem from conversations with NBIM.
} 
investments, it is the country weights that are adjusted. The equity share is typically higher for the listed- than the unlisted-real-estate benchmarks.

The benchmark returns differ considerably between the unlisted- and listed-real-estate portfolios, with an annualized average of $7.17 \%$ for unlisted compared to $10.35 \%$ for listed. The differences in the benchmark returns between the two portfolios is the main reason the average active return of the listed-real-estate portfolio is much lower (more negative) than for the unlisted-real-estate portfolio. So, if we were to evaluate the performance of the real-estate portfolios from their active returns, the listed-real-estate portfolio would seem to perform much worse than the unlisted.

As discussed in Section 2.2, one important property of benchmarks, according to Wermers (2011), is appropriateness. The real-estate benchmarks are appropriate in that they try to match the real estate's country, but the matching objects are from other asset classes. Thus, the Fund's benchmarks do not consider alternative real-estate investments' performance. Therefore, these benchmarks do not compare the Fund's real-estate investments to a similar investment universe. For this reason, we have placed little weight on the real-estate portfolios' active-return performance. It is interesting to compare the Fund's real-estate investments' performance directly with those of other real-estate investment managers. In NBIM's recent self-assessment (NBIM, 2021d), the Fund compares its unlisted-realestate investments against MSCI's IPD Global Property Index. After some adjustments (we have not checked them), the Fund finds that the unlisted-real-estate portfolio has yielded a lower return than the benchmark over the last five years. For the future, we encourage NBIM to consider reporting such analysis in more detail to the public.

\subsection{Summing up}

It is difficult to evaluate the performance of the Fund's real-estate portfolio with any precision for three reasons: the short sample period, the smoothed returns, and the inconsistent benchmarks.

The Sharpe ratio for the unlisted portfolio is larger than for the total portfolio, while it is smaller for the listed portfolio. The real-estate portfolio's costs are much larger than for its other investment strategies. The average gross returns of the unlisted and listed-real-estate portfolios are similar, whereas the average benchmark returns are much lower for the unlisted than for the listed portfolio. The average active returns for both the unlisted and listed portfolios are negative (but insignificant) and much smaller for the listed. The tracking error is large for both the unlisted and listed portfolios, and much higher than for the Fund's other investments.

The Fund has a fairly narrow focus in its real-estate portfolio, in that it is concentrated in a few large cities and a few sectors. This narrow strategy is reasonable because it helps reduce the costs and maintains local expertise. Over time, the Fund can gradually expand its focus. This benefit outweighs the potential diversification benefits from quickly expanding the unlisted-real-estate portfolio globally into many cities or sectors.

The Fund is very large, as are its real-estate holdings. Based on the previous research, we expect that the Fund has economies of scale with respect to the costs of its unlisted-real-estate investments. The previous literature shows that this is true for large pension funds, whereas this does not seem to be the case with respect to the returns (see Andonov, Kok and Eichholtz, 2013; Andonov, Eichholtz and Kok, 2015). Carlo, Eichholtz and Kok (2021) find that pension funds' investments in unlisted 
real estate have high costs. They find that European pension funds have average costs of $0.75 \%$ over their sample period, 1991-2018, which is both longer and earlier than our sample period. For the Fund's unlisted-real-estate portfolio, the average costs are $0.17 \%$ of the AUM, much lower than the pension funds' average. Figure 5 in Section 2.3 also shows that the costs for the unlisted real estate as a percentage are decreasing sharply over time, while Figure 32 in Appendix I shows that the AUM of unlisted real estate is increasing over time. Overall, the Fund seems to have economies of scale in its unlisted-real-estate investments. Still, the Fund might consider decreasing its investments in unlisted real estate in favor of listed real estate to further reduce its costs. We will come back to this suggestion and others related to real estate in Section 9.4. 


\section{Potential for value creation: Observations and suggestions}

This section discusses the Fund's potential for future value creation. We provide a list of observations based on our quantitative analysis in the previous sections and on our conversations with external experts, stakeholders, and NBIM and MoF representatives. In addition to a synthesis of the activereturn calculations and methodologies applied in previous sections, our observations include issues that may indirectly impact the Fund's potential to add value compared to its strategic benchmark. Based on these observations, we share a few key suggestions that could contribute to NBIM's future value creation.

\subsection{Evaluation of value creation}

Table 37 shows average annualized gross and net active returns and monthly value added for the Fund as a whole and separately for equity and fixed-income management. If an active return is positive and has a corresponding $p$-value below .05, we mark it with green. If it is positive and has a $p$-value above .05 , we give it a light-green background. Analogously, negative active returns are displayed in red or light-red. This table covers the full sample period (January 1998 to September 2021 except for equity, which begins in February 1998).

\begin{tabular}{lccc}
\hline & Fund & $\begin{array}{c}\text { Equity } \\
\text { management }\end{array}$ & $\begin{array}{c}\text { Fixed-income } \\
\text { management }\end{array}$ \\
\hline Gross active return & 0.28 & 0.47 & 0.17 \\
$p$-value & .21 & .01 & .71 \\
Net active return & 0.20 & 0.36 & 0.13 \\
$p$-value & .36 & .06 & .78 \\
\hline Gross value added & 0.80 & 0.77 & 0.13 \\
Net value added & 0.60 & 0.62 & 0.09 \\
\hline
\end{tabular}

Notes: The table summarizes the results from various other tables, showing the annualized means of the gross and net active returns and the gross and net value added. Returns expressed in percent. Value added is in NOK billion. Sample period: $1998-2021$.

Table 37: Fund summary of value creation

Since the start of the Fund, the annualized average net total return has been close to seven percent $(7.03 \%)$. Since the benchmark has shown an annualized return of $6.83 \%$, the average net active annualized return at the total Fund level is positive but small: $0.20 \%$. However, our analysis also shows that the corresponding $p$-value is high (.36), implying that the documented active returns at the total Fund level are not very likely to be different from zero. ${ }^{36}$

At the total equity-management level, net mean active returns are $0.36 \%$ with a $p$-value of .06 . In this setting without risk adjustment, equity management significantly creates value before costs: gross active returns are $0.47 \%$ ( $p$-value of .01). At the total fixed-income management level, net (gross) active returns are smaller: $0.13 \%(0.17 \%)$ with very high $p$-values.

The total accumulated value added for the full sample period is NOK 228 billion before and NOK 170 billion after costs. ${ }^{37}$ Furthermore, we find that almost all the value added for the total portfolio

\footnotetext{
${ }^{36}$ See Table 1 for more information on total returns and risks.

${ }^{37}$ With a NOK/USD exchange rate at 9, this is USD 25 billion before and USD 19 billion after costs.
} 


\begin{tabular}{|c|c|c|c|c|}
\hline & $\begin{array}{c}\text { Equity } \\
\text { management }\end{array}$ & $\begin{array}{l}\text { Fixed-income } \\
\text { management }\end{array}$ & $\begin{array}{l}\text { Real } \\
\text { estate }\end{array}$ & Fund \\
\hline Security selection & 1.27 & & & \\
\hline$p$-value & 0.04 & & & \\
\hline Internal & 0.93 & 0.36 & & \\
\hline$p$-value & .20 & .24 & & \\
\hline External & 2.15 & & & \\
\hline$p$-value & .00 & & & \\
\hline Enhanced indexing & 0.20 & 0.28 & & \\
\hline$p$-value & .00 & .00 & & \\
\hline Asset positioning & 0.13 & 0.25 & & \\
\hline$p$-value & .00 & .00 & & \\
\hline Lending & 0.07 & 0.03 & & \\
\hline$p$-value & .00 & .00 & & \\
\hline Fund allocation & -0.05 & -0.18 & & \\
\hline$p$-value & .66 & .11 & & \\
\hline Systematic factors & -0.04 & & & \\
\hline$p$-value & .59 & & & \\
\hline Environmental & 3.85 & 0.28 & & \\
\hline$p$-value & .08 & .17 & & \\
\hline Allocations & -0.05 & -0.18 & & \\
\hline$p$-value & .55 & .11 & & \\
\hline Unlisted real estate & & & -0.29 & \\
\hline$p$-value & & & .91 & \\
\hline Listed real estate & & & -3.85 & \\
\hline$p$-value & & & .48 & \\
\hline Total & 0.30 & 0.04 & -1.64 & 0.18 \\
\hline$p$-value & .11 & .78 & .56 & .14 \\
\hline
\end{tabular}

Notes: The table summarizes the results from various other tables, showing the annualized means of the net active returns. For enhanced indexing and fund allocation, we report gross active returns to avoid the allocation errors of the net returns. Returns expressed in percent. Value added is in NOK billion. Darker green indicates positive returns at or the $5 \%$ significance level, with lighter green for $p>.05$. Similarly, red indicates negative returns. Sample period: 2013-2021, except fixed income security selection (Oct 20142021), fixed income security lending (Nov 2015-2021), fixed income environmental (Jan 2016-Jun 2020) and real estate (2017-2021).

Table 38: Fund summary of substrategies' value creation

comes from the active equity management. ${ }^{38}$

Table 38 summarizes the active net returns and $p$-values of the substrategies for the recent sample period from January 2013 to September 2021. Studying the equity strategies results, we find some evidence of value-adding strategies. Security-selection strategies show positive active returns: $1.27 \%$ with a $p$-value of .04. The breakdown between internal and external security selection shows that this positive result can likely be attributed to external management strategies $(2.15 \%$ with a $p$-value of .00). Although internal security selection has a positive net active return of $0.93 \%$, again before risk-adjustment, the corresponding $p$-value (.20) shows that it is not statistically significant. The enhanced-indexing investment strategies (asset positioning and security lending) provide small (in

\footnotetext{
${ }^{38}$ Note that the sum of equity and fixed-income value added does not add to the Fund value added. This is mainly due to the negative value added for real estate. Even though real estate has only been around as an active strategy for five years, Table 63 shows that the negative active contribution has been substantial.
} 
total $0.20 \%$ ) but consistently positive alphas (with $p$-values close to zero). ${ }^{39}$ The third subcategory in equity strategies, fund allocation, is very difficult to assess as it is an amalgam of many diverse strategies. Nonetheless, based on our results, it is safe to conclude that fund-allocation strategies, at the total level, do not provide positive active returns.

Inspection of the fixed-income strategies shows a similar picture for enhanced-indexing strategies (asset positioning and security lending). Active returns are positive (0.28\%), and $p$-values are again close to zero. However, active returns of the security-selection and allocation strategies do not appear significantly different from zero (given the high $p$-values of .24 and .11, respectively).

Even if results for real estate are reported, we are very cautious about drawing any conclusions about performance given the issues discussed in Section 8. However, it is clear that given this benchmark setting, realized active returns are negative but insignificant.

In sum, our calculations without risk adjustment show that active returns at the total Fund level are small and most likely not different from zero. Some investment strategies seem to provide significantly positive active returns: external security selection (equity), asset positioning (equity and fixed income), and security lending (equity and fixed income).

The small active returns can be attributed to our finding that tracking errors are generally very low (with a negative trend, see Figure 10), both at the total Fund level and at the individual substrategy level. As discussed at the end of Section 3.2, it is not clear to us whether this is caused by constraints due to the Fund's size (see, for instance, Andonov et al., 2012, who show that it is very difficult for large pension funds to increase activeness in public markets without negatively impacting alphas, even for funds that are considerably smaller than the Fund), risk aversion by the NBIM's leadership (see, e.g., Cremers et al., 2019, who show that funds that stay close to the benchmark generally underperform), a lack of opportunities (as a result of mandate, capacity, or budget constraints), a combination of these factors, or something else entirely. Interestingly, in the context of equity markets, Dyck et al. (2013) find that benefits from active management are the highest in markets where potential deviations from fundamental values are likely to be the largest and potential competition lowest (such as emerging markets).

\section{Suggestions}

- We encourage the MoF to provide NBIM with a mandate that contains a clear active-return target. The target could serve as guidance for NBIM's strategic decision making regarding its active management. Further, it would give future review committees a better basis for their feedback.

- We advise the MoF to investigate why NBIM does not take full advantage of its tracking-error limit. The objective of this study would be to understand why this ex-ante limit is not reached, whether any operational impediments or structural barriers play a role, and whether and how they can be lifted, if relevant. We are especially interested in how the tracking error of successful strategies (e.g., equity asset positioning) can be increased. In addition, we suggest NBIM share its thoughts about its active risk position more explicitly and proactively with the public.

\footnotetext{
${ }^{39}$ The enhanced-indexing and fund-allocation strategies are displayed in terms of gross returns because the two have only small differences between gross and net results.
} 
- Since the Fund is very large, we also advise investigating the potential presence of (dis)economies of scale in NBIM's ability to achieve active returns. Large funds have substantial economies of scale regarding investment costs (Andonov et al., 2012), but they simultaneously have difficulties in beating equity benchmarks because of diseconomies of scale in achieving active returns (related to liquidity constraints, market impact, and other matters with a larger impact than the cost advantages). It would be interesting to investigate further which assets, strategies, and substrategies are most affected by these issues. For instance, it may be that the successful strategies (e.g., enhanced indexing) are most affected by liquidity constraints or limited opportunities.

- Using input from a thorough assessment of potential barriers and diseconomies of scale, we recommend considering allocating more active-risk budget to those strategies that have consistently outperformed, potentially at the expense of strategies that do not seem to add any value. This assessment could also result in an adjustment of the ex-ante tracking-error limit.

- We recommend further investigation of the underlying reasons for the consistent success (very high monthly hit ratios) of the enhanced-indexing strategies in equity and fixed income in the postcrisis period (2013-2021). This could include an investigation into potential risks that cannot be captured by the empirical section's methodologies (such as operational risk, counterparty risk for security lending, or asymmetric distributions of active returns).

\subsection{Risk-adjusted performance evaluation}

As we wrote in Section 3.4, it is a sheer impossible task to choose a risk model that encompasses the Fund's full set of investment strategies and substrategies and the underlying historical dynamics (such as recent benchmark changes, starting and stopping factor-tilting strategies, and investing outside the Fund's benchmark universe in various asset categories) in the period under investigation. The dynamic nature of the investment and benchmarking process would require a risk-adjustment procedure able to capture time-varying factor exposures (for instance, along the lines of Ferson and Schadt, 1996). Moreover, some of the ad hoc fund allocations (such as duration and beta plays in both directions, exposure to emerging-market fixed income, and investments in China A shares) would be very difficult to capture properly in any one model. It would require constructing a dynamic factor model that is specific for the structure and history of the Fund. We feel that this would not reduce but increase complexity and so would not contribute positively to NBIM's accountability to the MoF and the Norwegian public.

This notion explains our decision to use a simple CAPM framework as a base case for risk adjustment and the well-known and widely applied Fama and French (2015) factor model (the five-factor model extended with two additional factors for fixed income), also advocated by Dahlquist et al. (2015). This method gives us preliminary quantitative insights, but we are aware of potential model and parameter uncertainty with any of these performance-evaluation models.

First, the universes underlying these factors fully match neither the Fund's benchmark universes 
nor the Fund's portfolio universes. This will create a biased alpha estimate. ${ }^{40}$

In addition, as mentioned before, Akey et al. (2021) show that using Fama and French (2015) data in multifactor models is also not without measurement issues as factor returns differ substantially depending on when the data is accessed. The effects of these retroactive changes on active-performance measures can be large. The potential "noise" resulting from the vintage choice of the factors is much larger than a potential "signal" (the active return).

The above discussion shows that any factor model will be prone to omitted factors and other issues that could contribute to model and parameter uncertainty. Hence, we must be careful when interpreting any of the active returns (including those without risk adjustment). Therefore, we base our review not solely on quantitative, but also qualitative considerations, especially given the near-zero active-return numbers in Table 37.

The conclusion based on risk-adjusted results is that net alphas from these regressions are very small at the total Fund level and the full sample period: $0.06 \%$ with high $p$-values (see Tables 5 and 6 ). For the basic performance comparison with the benchmark, risk-adjusted alphas are also somewhat small at the equity management level: $0.25 \%$ (for both the CAPM, Table 10 and the Fama and French (2015) five-factor model, Table 11) with $p$-values larger than .10. At the fixed-income management level, alphas are again small and not significantly different from zero (see Tables 23 and 24). In general, alphas are higher for the last sample period (2017--2021).

The risk-adjusted alphas for substrategies are generally close to those in the basic comparison: Alphas for external security selection and enhanced indexing (asset positioning and security lending) for equity and fixed income are significantly different from zero. We also find that the mean active return of equity internal security selection decreases considerably after risk-adjustment (with an estimated beta equal to 1.05 , see Table 14).

\section{Suggestions}

- We advise evaluating NBIM's active management with simple, understandable, and widely accepted performance-evaluation models. For this reason, we apply three well-known methods to evaluate performance as a base for this review: Basic performance comparison (with standard deviations), CAPM-based risk adjustment, and a multifactor approach along the lines of Dahlquist et al. (2015).

- We deem the fine-tuning of performance-evaluation methods to the Fund's past and future context a sheer impossible task given the differences in portfolio and benchmark universes, time-variation in factor exposures, the complexity associated with benchmarks, historical benchmark changes in the mandate, and several changes in the management team.

- Instead, in our view, future review teams should apply more qualitative elements in their feedback process. That implies having more interviews and site visits and, at the substrategy level, a better understanding of the strengths and weaknesses of NBIM's current organization. That way,

\footnotetext{
${ }^{40}$ Other factors may explain realized returns. For example, Pastor et al. (2021a) and Pástor, Stambaugh and Taylor (2021b) propose a two-factor model with a green factor based on the return spread between environmentally friendly and unfriendly stocks. This factor also seems to explain the recent underperformance of value stocks in Pastor et al. (2021a): "Realized returns are a popular proxy for expected returns in the empirical asset pricing literature. However, high realized returns do not always indicate high expected returns, especially if they are realized over a relatively short period."
} 
more will be learned of relevant organizational and institutional factors impacting the potential for future value creation at the total Fund level and for individual assets.

- Finally, the MoF should consider updating Section 2.4 (paragraph 7) in NBIM's factor exposure mandate (Ministry of Finance, 2021). It is not clear how NBIM should act based on this statement. For example, to which factors should the Fund be exposed?

\subsection{A complex structure}

Despite its low active risk profile, NBIM's organization runs more than 200 investment strategies and substrategies. NBIM reports on these strategies to the outside world at a higher aggregation level, quite like Table 37 in our first observation in Section 9.1. As a review committee, we are the first to provide detailed insights on the key substrategies. Providing a full overview of all substrategies is not possible as the number of individual substrategies (and subsubstrategies below this level) is simply too large. In addition, the time commitment and resources that come with the review committee's mandate do not allow us to investigate all strategies in detail.

NBIM has been very helpful, delivering information and providing background on their investments (performance, costs, and other related information such as voting and engagement overviews). Moreover, we feel that NBIM generally reports transparently on its investments to the public. Nonetheless, it has been quite a challenge for us to understand the details of NBIM's investment organization, its key investment strategies, and especially its substrategies. We did not expect this to be the case, given the low tracking error versus the mandate at the total Fund level and the abundance of information available in the public domain.

NBIM reports on an aggregated level showing three distinct strategies: asset management (we name it enhanced indexing), security selection, and fund allocation, thereby spanning two asset classes and encompassing six key investment strategies. In addition, they document listed and unlisted-real-estate portfolios. Although the subdivision in Table 38 is quite straightforward, it also shows part of the complexity. For instance, the performance of the fund-allocation category is the result of several decisions made in the past or that are presently prevalent in the portfolio exposure. Examples are the impact of (past) factor strategies, the impact of real estate on the active performance, the impact of the MoF-mandated environmental portfolios, and "allocations," which is a container category of strategic, tactical, and other allocations: duration plays, beta plays, emerging-market fixed income, infrastructure investments, China A-share exposure, and so on. Another example is the environmentalstrategies mandate. Now it is labeled under the fund-allocation umbrella. It may fit better among the internal-security-selection strategies.

It is not always clear to us how the performance information NBIM reports to the public (see Tables 37 and 38) can be mapped to the Fund's investment departments and portfolio-management teams. Team responsibilities are not directly mapped to the cells in this matrix, which makes it very difficult for outsiders to discern which (sub)strategies performed well and, importantly, what can be learned from this for the future. It also complicates reporting to various stakeholders. In our view, the observed complexity reduces the possibility for outside stakeholders (including well-intentioned academics) to provide meaningful feedback.

As described in Section 2.2, we do not have full clarity on the internal benchmarks' construction 
for strategies and, especially, substrategies and the building blocks of these strategies in levels below (for instance the performance of sector teams). This lack of transparency will impact the possibility of meaningfully reviewing substrategies. The previous review committee also made this comment. An example is that the real-estate team currently has two benchmarks: one based on the funding/financing decision (see Section 8) and one that more resembles a public real-estate benchmark index.

Given that the received return and cost data (for portfolios and benchmarks) are internally and externally audited, we feel confident that the documented net active return of $0.20 \%$ (positive) is correct. ${ }^{41}$ We can therefore conclude that the observed total performance of the Fund resembles its benchmark closely. Similarly, we can make a good assessment of the Fund's performance at the equity and fixed-income management levels and their key substrategies. However, with the information available to us, it is difficult to investigate at more granular levels which parts of the organization are performing well and which are not. Given the observed complexity and many other confounding factors (mismatched portfolio and benchmark universes, lack of clarity about benchmarks in some instances, the impact of imposed benchmark changes, potential measurement issues because of tax reclaims, and simply the model and parameter uncertainty of models trying to capture the, potentially time-varying, risk of the total portfolio), we feel that we must be cautious about giving too much weight to any of the numbers presented. ${ }^{42}$

\section{Suggestions}

- We advise NBIM to better explain how investments can be mapped to teams and departments. More insight into this will help future review committees and the general public understand which parts of the organization perform well and which do not.

- We are the first review committee to investigate active returns at the substrategy level (Table 38). Future review committees could study in more detail why some of these strategies work well and some do not. For instance, is the consistently positive performance of asset positioning the result of a particular subsubstrategy (e.g., index-inclusion strategies) or is it a mix of different positive strategies? ${ }^{43}$ High hit ratios (see Table 49) make us curious regarding the benchmark design. It may also be that these strategies face tail risks uncaptured by our methodology (extreme "black swan" events). Better documentation of substrategies can rule out other circumstances' positive performance impact, which will also contribute to accountability to the Norwegian public.

- In a similar vein, we encourage NBIM to extend the sample period for which data is available for substrategies in equity and fixed income. That would provide stakeholders more insights into how active management performs in times of crisis (such as the burst of the dot.com bubble and the recent financial crisis and its aftermath). If that is not possible, we ask NBIM to provide a clear explanation to future review committees and to all stakeholders in general why this is not possible.

\footnotetext{
${ }^{41}$ We rely on high-quality internal and external auditing and assurance. We are not able to check the quality of the data provided, both because of time constraints and the complexity of the operations.

${ }^{42}$ NBIM kindly provided us with much detail on the withholding tax reclaim process and how it is integrated into the benchmarking adjustment. Given this information, we conclude that this information is integrated into the benchmarks correctly.

${ }^{43}$ NBIM (2020c) is a good start, but room remains for a more pedagogical description.
} 
- Similarly, the internal benchmarking process (especially for but not restricted to substrategies) is very unclear to us, as it was for Dahlquist and Ødegaard (2018). Again, more clarity about the benchmarking of active strategies will lead to more rigor in evaluating strategies and substrategies. Hence, we suggest that NBIM makes the benchmarking process more transparent to the public.

- The benchmarking process for real estate is particularly unclear to us and probably does not support the real-estate investment process. Trying to beat two benchmarks is a very unsatisfying assignment for all involved. More on this in the next subsection.

- Another issue we would like to raise is the allocation of costs to individual investment strategies or substrategies. We take the cost information that we received from NBIM as a given. However, it may be of interest to investigate further if costs are allocated properly to individual strategies and substrategies as, potentially, some strategies could be (implicitly) cross-subsidized by others. Having more information on costs for individual strategies, also back in time, will enhance the quality of the strategy and substrategy evaluation. For example, in our analysis of internalsecurity-selection strategies, we did not receive cost information for equity and fixed income separately. Detailed cost information (at a granular level) could also be made publicly available.

\subsection{Potential conflicts of interest in NBIM's mandate}

The total returns delivered by the Fund will be largely determined by the choice of the benchmark by the MoF. Since its inception in 1998, the Fund's annualized average net total return (after costs) is around $7 \%$, whereas the average active return (versus the benchmark) is around $0.20 \%$. These numbers show the relative importance of the strategic decision-making process versus the active-management process (Brinson et al., 1995).

NBIM's organization understandably seems to be focused on the key elements of the MoF's mandate: To deliver as large an active return as possible in predominantly public markets (equity, fixed income, and real estate) within the mandated risk limits. The mandate also states that NBIM should provide advice to the MoF in matters related to the Fund's strategic direction.

However, the strategic investment direction (including strategic asset allocation) is not determined by NBIM. Mandate updates and resulting benchmark changes will occur from time to time. Good examples are the inclusion of real estate and emerging-market fixed income in the benchmark and the subsequent removal of the same asset categories.

Changes in the Fund's investments due to changes in the benchmark regarding liquid, public assets (such as a more global exposure to equity instead of an overweight in Europe) are relatively easy to execute and associated with relatively low trading costs and organizational change. Benchmark changes that involve starting up or winding down illiquid investments are more complex, organizationally impactful, and expensive.

In general, any benchmark change will lead to portfolio reallocations and trading-strategy adjustments and, as a result, will be associated with additional (trading and other) costs. ${ }^{44}$ As mentioned above, these changes potentially also lead to organizational challenges. For example, expertise built up to manage (unlisted) real-estate portfolios had been hired since the inclusion of real estate in the

\footnotetext{
${ }^{44}$ Section 2.2 described the Fund's estimated cost for inflows and extraordinary benchmark changes and how we dealt with that in connection with the performance analysis.
} 
benchmark and the portfolio. Targeted human capital had been attracted to take on the management of the unlisted-real-estate portfolio. By formally removing real estate from the benchmark in 2017, the MoF indirectly signaled that NBIM will not be able to build up a sizable, long-term stake in unlisted real estate. From that moment onwards, the Fund's exposure to real-estate operations will be bound by the tracking error of the mandate at the total level.

This indirect signaling may impact NBIM's ability to attract and retain key personnel, especially in the context of the unlisted-real-estate and infrastructure team. Building up private-market teams usually takes many years, but if key personnel does not see the long-term viability of their contribution to the organization (as real estate is not part of the benchmark) and face career concerns, they may focus on short-term investment opportunities (see van Binsbergen, Han, Ruan and Xing, 2021) or leave in search of jobs in which they can use their skills, experience, and knowledge more fully. The same logic holds for other, more complex investments such as infrastructure and private equity. Setting up these investment teams requires a long horizon. ${ }^{45}$

Investing in certain private or complex asset categories not part of the Fund's benchmark also comes with another major challenge. We want to illustrate this using the real-estate context.

Despite the removal of real estate from the benchmark in 2017, NBIM was allowed to continue investing in unlisted and listed real estate (and encouraged to start up infrastructure investments). These investments would be interpreted as active deviations from the benchmark mentioned in the mandate. Going forward, these investments would compete for parts of the active risk budget with other investment strategies in the Fund. In our conversations with NBIM, we were told that real estate, despite its exclusion from the benchmark, continues to have a diversification role. ${ }^{46}$ We fully agree that adding real estate (as a mix of unlisted and listed) to the strategic mix will probably lead to lower risk for the portfolio (and a higher Sharpe ratio). However, the optimal allocation to real estate will most likely differ from the current allocation as this is bound by the mandate's tracking error. ${ }^{47}$

In our view, this context gives rise to potential conflicting objectives and inefficiencies in a few dimensions. NBIM, in fact, has two objectives.

- To invest in real estate as a diversifier (in the total return-risk space)

- To develop real-estate investment strategies as a source of active return versus the mandate's benchmark (in the active return-risk space)

Implicitly, this means that the Fund has two key objectives: Achieving the highest possible Sharpe ratio and achieving the highest possible $I R$. However, the Fund has just one instrument: the (realestate) investment portfolio. This construct seems to be at odds with the Tinbergen rule (transferred from macroeconomics to investments and paraphrased): For each objective, one needs to have one instrument (see Tinbergen, 1952). These potentially conflicting objectives could lead to organizational challenges for the Fund. From a Sharpe-ratio perspective, it may be wise to continue to invest in real estate, but it may be wise to deploy the organizational focus and tracking-error budget on other value-adding strategies from an $I R$ perspective, such as the successful enhanced-indexing strategies.

\footnotetext{
${ }^{45}$ This is also in line with one of the recommendations from Døskeland and Stromberg (2018) should NBIM enter private-equity investments.

${ }^{46}$ This is consistent with the description of the Fund's real-estate investment strategy in NBIM (2020d).

${ }^{47}$ Note that liquidity, related to the Fund's size, also has an impact on the optimal allocation. There may be an upper limit on the real-estate investments.
} 


\section{Suggestions}

Although the definition of NBIM's mandate and the associated benchmark composition is only indirectly part of our mandate, we suggest evaluating NBIM's current mandate along the following lines.

- Clarity about NBIM's mandate and its value-creation potential is key for all parties involved. If real estate is indeed expected to be in the portfolio for strategic reasons, it also needs to be represented in the strategic benchmark. That way, NBIM can attract and retain human capital able to deliver on this strategic objective. The resulting real-estate portfolio could be (as it is now) a mix of listed- and unlisted-real-estate investments. As the team grows, the portfolio would also be even more diversified across the globe and, as such, contribute more to the diversification objective (and hence increased Sharpe ratio).

- If the MoF's decision to remove real estate from the benchmark still stands, we would argue that NBIM should consider gradually winding down the unlisted-real-estate investments. As time passes, it will most likely become increasingly difficult to attract and retain the required human capital to manage and monitor these private-market portfolios professionally, with a potential negative impact on the portfolio's performance.

- Note that removing the unlisted-real-estate portfolio would have a small impact on total portfolio characteristics as the portion invested in unlisted real estate $(2.5 \%)$ is relatively small, while the size of the team to run such a complex operation is relatively large; complexity is also much larger than in public investments, which also impacts NBIM's control and compliance departments more than proportionally, and similarly, the potential to deliver high-quality accountability to the public.

- If the unlisted real estate will remain out of the benchmark, it may still make sense to continue to invest in listed real estate, as it can be easily globally diversified and managed by a relatively small team. Potentially, the listed-real-estate allocation could be part of the strategic benchmark (using an appropriate global listed-real-estate benchmark, which would also make the benchmarking process more transparent and coherent). The strategic weight could be a function of the size and depth of these markets. Alternatively, the Fund's benchmark could exclude all real-estate investments, and NBIM could invest in listed real estate merely through its positions in the equity benchmark (as real estate is a small sector in the MSCI world index). It could then be considered as an active sector bet, as it is now.

- In principle, the same logic holds for infrastructure investments. Infrastructure investments are very complicated investments in which every single investment has different risk and return characteristics and specific governance challenges. This complex context requires the setup of a seasoned infrastructure team that can adequately select and monitor these investments. We would argue that not having infrastructure in the Fund's strategic mandate will lead to the same issues as in the case of unlisted real estate.

- There may be a few other (but minor) related issues in the public-investments space. For instance, the Fund has positions in China A shares (previously not in the benchmark) and emerging-market fixed income, and runs environmental mandates, which are not part of the benchmark (but are mandated by MoF). This is an example of the Fund's investment universe not overlapping with its benchmark universe, as discussed in Section 2.2 and also by Wermers (2011). This difference 
will continue to lead to questions about the source of active returns and related risk-adjustment procedures. A potential solution is regularly calibrating and updating equity benchmarks and reporting on them. Note, a tension always exists between active portfolio managers and those who evaluate them regarding benchmark choice. If the universe of equity or assets beyond the benchmark is high, opportunities abound, by construction, to beat that benchmark (given a skill level). In our opinion, benchmarks must reflect the relevant and investable assets available in the public-market space. ${ }^{48}$

- In sum, our suggestions contribute to creating a clearer mandate for NBIM, which is in the long-term interest of the Norwegian people (legitimacy), the MoF (accountability), and NBIM itself (focus on achieving key objectives).

\subsection{Active ownership and responsible investments}

In this observation, we explain why decisions in NBIM's mandate on how, and with what level of intensity, to execute active ownership strategies (including the recent focus on climate change), which may impact the Fund's potential (positive and negative) to provide active returns versus the benchmark in its mandate.

A review of NBIM's stewardship and active-ownership policies and implementation is not an explicit part of our mandate. However, we argue that further integrating ESG (environmental, social, and governance) information into the Fund's investment strategy could impact NBIM's potential to conduct active management strategies. Increased and intensified efforts in active ownership strategies as well as more explicit integration of sustainability-related information into the portfolio-management process (which could have various incarnations, such as divestments, integration of ESG-information into the portfolio-management and selection process, and impact investments) may require more budget to prepare, execute, and monitor these activities. It will also require more knowledge and experience in the domain of responsible investments, and it may be associated with potential changes in benchmarks and the mandate (such as shifting to benchmarks that already consider climate change when determining index constituents). These developments may require a different focus in the investment strategy. As such, they could compete with resources now deployed in other investment strategies.

NBIM's current activities in the field of responsible investments are well documented in its 2020 responsible-investments report (NBIM, 2020j). This report shows that NBIM takes its role as a responsible asset owner very seriously. In 2020, NBIM voted on $98 \%$ of the shareholder meetings of portfolio companies. Moreover, NBIM had 2,877 company meetings and 650 written communications with companies on various matters related to responsible investments.

The responsible-investments report also shows that NBIM divests from companies for ethical or sustainability reasons (see NBIM, 2020j, p. 82, Divestments). In 2020, NBIM excluded 15 companies, revoked the exclusion of three companies, and placed a further four companies under observation. ${ }^{49}$

\footnotetext{
${ }^{48}$ This is similar to what van Binsbergen and Koijen (2018) recommend regarding the fixed-income benchmark. One could argue that, from the perspective of the portfolio manager, the call option (possibility to get outperformance) will be less valuable when the number of investable opportunities outside the benchmark decreases.

${ }^{49}$ Until 2015, the MoF made decisions on exclusion and observation of individual companies, based on recommendations from the Council on Ethics for the Fund. From 2015, the MoF has delegated these decisions to the Norges Bank executive board based on recommendations from the Council on Ethics (except for coal exclusions, which are identified by Norges Bank). When the executive board decides to exclude a company, it informs the MoF and the MoF decides the date when the company is removed from the benchmark.
} 
Next to the 15 ethical exclusions, NBIM also excludes 106 companies that produce certain types of weapons, tobacco, coal, or use coal for power production. Furthermore, 38 companies are currently excluded because of "an unacceptable risk of them contributing to or being responsible for particularly serious violations of fundamental ethical norms." The benchmark accounts for these exclusions.

Next to these exclusions, NBIM also divested from 32 companies following assessments of ESG risks (314 companies since 2012). The report further states (page 86) that "the names of companies subject to risk-based divestments are not disclosed, but NBIM does publish a list of its holdings on its website each year" (NBIM, 2020j, p. 86). Note that the Fund's benchmark does not account for these divestments. Finally, the responsible-investments report also shows close to NOK 100 billion invested in in-house environmental investments in 2020.

In March 2021, the MoF asked NBIM to assess and present alternative ways of addressing financial climate risk and climate-related investment opportunities in the Fund's management (letter only in Norwegian Minstry of Finance, 2021). In a reply (July 2021), NBIM (2021a) sketches alternative ways of addressing climate risk within the current mandate. Subsequently, they discuss implementation paths for which the current mandate needs to be amended. In its response, NBIM states the belief that there are no grounds to suspect that climate risk is systematically mispriced. In addition, they state that "major changes to the principles underlying the Fund's investment strategy should be made with caution."

In contrast to these beliefs, NBIM also states in the letter that their portfolio managers consider climate risk and climate-related investment opportunities before deciding to invest in individual companies. They add: "Such adjustments require proximity to, and familiarity with the markets, and their implementation should therefore be delegated to Norges Bank as it is today. Through our contributions to standard setting, clear expectations, dialogue with companies and voting, we will seek to ensure that the companies in our portfolio are well-equipped for the low-carbon transition."

Almost in parallel, the MoF appointed a climate-risk expert group chaired by Martin Skancke on "Climate risk and the Government Pension Fund Global" (Skancke et al., 2021). This expert group presented its report in August 2021. The group advised that NBIM's current management mandate should better reflect the importance of climate risk. They propose that NBIM establish a set of principles for the measurement and management of climate risk. Moreover, they advise that NBIM should be guided by a "long-term target of zero emissions of GHG from the companies in the investment portfolio, in line with international climate agreements to which Norway has acceded." They agree with the current investment strategy that is characterized by the broadest possible diversification as this is the first line of defense for a large fund such as the Fund. At the same time, they advise that "responsible investment and active ownership should be strengthened and the requirements for measuring, managing and reporting climate risk increased."

The climate-risk expert group expects that the Fund, through a prominent deployment of active ownership tools (see Box 7.4 in their report), can test and potentially influence the robustness of business models of the companies in which the Fund has invested. Active ownership may also contribute to

\footnotetext{
${ }^{50}$ Targeting a nonfinancial objective (net-zero GHG emissions) seems to be at odds with the statement made by NBIM: "Our motivation for responsible investment is to achieve the highest possible return with moderate risk. Companies' activities have a considerable impact on society and the environment around them. Over time, this could affect their profitability and so the Fund's return. We therefore consider both governance and sustainability issues and publish clear expectations of companies in the portfolio." https://www.nbim.no/en/the-fund/responsible-investment/
} 
companies channeling more capital to profitable projects in the transition to a low-carbon economy. Active ownership may, according to the expert group, also strengthen financial markets' ability to price climate risks (through better reporting). They also add that failed engagement may eventually lead to divestment from companies. ${ }^{51}$

Based on the above, we analyze how an increased focus on active ownership strategies (including climate change), could impact NBIM's potential to achieve active returns. We rewrite this into two key categories: different objectives, potentially leading to conflicts of interest, and conditions for a successful implementation of active-ownership strategies.

\section{Different objectives, potentially leading to conflicting goals}

First, targeting companies through active ownership strategies (engagement, voting, threat of divestments, and more) has the objective to let companies perform better, and as such is targeted at total returns (in contrast to active returns). If NBIM engages with a chemical company to upgrade its environmental-management system to encourage the company to be better prepared for future legislation changes, this may (if the engagement is successful), have a positive impact on the company's stock price in the long run (see Dimson, Karakaş and Li, 2015). This is especially the case when engagements are conducted in coordination with other institutional investors (see Dimson, Karakaş and Li, 2021). If the engagement is successful, all investors in that company, including those who did not engage, will profit from the rise in the stock price. This shows that engagement mainly targets companies with upside potential on ESG topics (and potentially even beyond).

Now, suppose that NBIM has invested in this chemical company, but with a weight lower than the benchmark weight (for instance, because the security selection team has doubts on the viability of its general business model). Successfully engaging with this company will increase the return of the Fund's portfolio (ceteris paribus), but the active return versus the benchmark will be negatively affected as the weight versus the benchmark is negative: if the company performs well, it hurts alpha. Similar examples of potentially conflicting goals can be framed for other active-ownership strategies such as filing shareholder proposals, starting class-action lawsuits, and proxy voting.

This example shows that the objective to intensify and extend the active ownership effort may be at odds with the objective to harvest active returns. It also raises the question of who decides which objective is prioritized in which context. In essence, this is another example of having one instrument (the Fund's investment portfolio) and two objectives (active-ownership impact and active return).

The climate-risk expert group's statement that NBIM should be guided by international climate agreements to which Norway has acceded raises another conflict of interest. Focusing on reaching long-term zero-emission targets may be at odds with fully using the potential to harvest active returns. For instance, it may be that, in the eyes of portfolio managers, fossil fuel companies are undervalued (when looked through a long-term lens). Hence, they propose a substantial overweight in (some of) these companies. That proposal could be inconsistent with a long-term zero-emission target. Again, this example shows that there are potential conflicts of interest. NBIM's mandate should give guidance on which decisions are to be prioritized. Again, NBIM would have two objectives (zero-emission targets

\footnotetext{
${ }^{51}$ In December 2021, NBIM released two letters to MoF. The first comments on the Skancke report (NBIM, 2021f), while the second describes its experience with a climate criterion (NBIM, 2021b).
} 
and active returns or put differently: nonpecuniary and pecuniary targets) and just one instrument (the Fund's investment portfolio).

\section{Conditions for a successful implementation of active-ownership strategies}

Intensifying and extending active ownership along the lines advocated by the climate-risk expert group could imply deploying many additional resources: Attracting additional human capital to execute these strategies, setting up or extending systems to monitor and report progress to the public, participating in collaborative vehicles, and potentially also to hiring additional external services to support the execution. This development will materially affect the Fund's budget. If this budget partially needs to be financed by redeploying resources in the current budget, it may affect resources spent on other (active-management) activities.

Credibly and successfully engaging with company management on material ESG issues requires the ability to engage in conversations with company management, sector-specific knowledge of business models, knowledge of (sector-specific and beyond) ESG material issues, and knowledge of activeownership tools.

This shows that the execution of these strategies requires an investment in or redeployment of human capital. In this context, we appreciate NBIM's experience in engaging with companies. The combined knowledge and expertise of the governance and internal-security-selection teams ensure credible access to company management, seniority, and experience in engaging with companies on all matters (including but not restricted to climate change). We are not sure, though, whether the task related to increased and intensified active ownership in the whole portfolio, as proposed by the climate-risk expert group, can be executed with the current team's capacity. The team that runs the (relatively small) environmental mandates could interact with and support the team responsible for active-ownership strategies more than before. This requires an extended coordination effort among the portfolio-management and active-ownership teams. ${ }^{52}$

Active-ownership strategies require that those who execute the strategy have a long-horizon mindset and a greater focus than before on material issues in the ESG space. The same logic holds for an increased emphasis on adequate climate-risk reporting by companies. Currently, many of the activities in enhanced indexing and asset positioning (equity and fixed income) are short-term oriented. Portfolio managers in this domain most likely will have less experience in dealing with long-term topics related to climate risk and similar matters. The internal-security-selection team seems to have a long-term (sector-specific) focus when determining the selection of stocks in the portfolio, but it is not fully clear to us how much weight ESG-related risk and opportunities (including climate) get in the decision making. The environmental-mandates team's focus is probably fully aligned with the objectives of the extended engagement effort. In our conversation with a representative of the environmental team, we learned that the team regularly exchanges information on companies with the security-selection team. However, since the environmental mandates strictly invest in a selection of high-performing ESG companies, they will not be able to comment on all stocks in the portfolio (especially those with considerable ESG upside potential).

\footnotetext{
${ }^{52}$ In our view, increasing and intensifying active ownership will be more successful if NBIM joins additional collaborative frameworks with similar goals as described in the climate-risk expert group (such as Climate Action 100+) but this may be restricted by Norway's sovereignty.
} 
We also add the notion that the active-management teams focus on a selection of the stocks in the Fund's portfolio, and active ownership strategy involves interacting with other stocks in the portfolio. The active-management teams are unlikely to have the same insight on these companies as those that are part of the most prominent active positions. Finally, it is not clear to us how external managers could be aligned with the ownership strategies and whether this could lead to potential execution problems. Creating a culture that contributes to successful active ownership strategies will be an important task for NBIM's leadership, while at the same time running the investment portfolios.

When the MoF decides how to integrate the climate-risk expert group's recommendations in the mandate, it should be clear how the different objectives will be prioritized, which targets should be achieved, and how incentive schemes are compatible with these priorities. The prioritization also involves deciding how much effort to put into the different ways that active ownership can be executed. Moreover, the Fund's mandate should be structured to avoid or minimize conflicting priorities.

\section{Suggestions}

Based on this analysis, we offer four suggestions.

- The MoF should provide clarity in NBIM's mandate regarding objectives and prioritization of active-ownership strategies, as well as clarity on what is delegated to NBIM and what is contained in its mandate. Especially in the case that an additional objective is defined (e.g., long-term zero-emission targets for the companies in the Fund's portfolio), clarity on the governance and objectives of the active-ownership process is crucial.

- The MoF should provide clarity on NBIM's budget: Will extra resources spent on activeownership strategies lead to an increase in NBIM's budget, or will NBIM have to (partially) redeploy resources?

- We agree with the climate-risk committee (Skancke et al., 2021) and suggest that the MoF consider integrating the environmental mandate into the general investment process (people and portfolios).

- If the MoF decides to increase and intensify active ownership (on climate risk), we advise NBIM to create a human-capital strategy that supports this direction. This will involve ensuring that employees executing the active-ownership strategies have the necessary long-term mindset, supporting the exchange of relevant ESG information, and fostering a company culture embracing the increased focus on ESG issues. In our conversation with NBIM, we have learned that the environmental-mandates team (with a relatively small mandate) does exchange information and knowledge on environmental issues with other active-management teams within NBIM. Moreover, the internal-security-selection team can deploy their experience and skills in active-ownership strategies. However, these positive externalities may be offset, at least to some extent, by potential conflicts between active-ownership objectives and active-management aspirations. 


\section{Concluding comments}

At the start of this report, our executive summary provides a full overview of the context, the mandate, our approach, and our key findings and interpretations of these findings. In this section, we summarize our key observations and suggestions.

\section{Key observations}

- The performance of the Fund at the total level is slightly higher than its benchmark $(0.20 \%)$. However, this positive active return is not statistically significant. Some substrategies at lower aggregation levels seem to provide significantly positive active returns, such as enhanced indexing (both equity and fixed income) and external equity security selection.

- Risk-adjusting the active return series does not lead to materially new insights on NBIM's performance. Results are very similar when compared to the basic performance analysis.

- The Fund's key attributes on risk and return show that it is, in essence, an (enhanced) index fund. Nonetheless, the Fund is managed in a very complex way. This complexity implies that we can evaluate properly at the total Fund, equity, and fixed-income level, and we are also able to give feedback at the key substrategy level. But better understanding why some substrategies work and others do not requires more granular information on individual substrategies.

- The current benchmark gives rise to some potential conflicts of interest as some objectives target active returns (trying to achieve a positive alpha) and others target total returns (diversification of the whole portfolio). A future MoF decision to add net-zero-emissions targets to the portfolio would add more conflicts of interest to the mandate.

- Currently, across the globe, many developments in active ownership and responsible investments are arising. This could impact the potential and direction for active management going forward.

\section{Key suggestions}

- We encourage the MoF to provide NBIM with a mandate with a clear active-return target. The target could serve as guidance for NBIM's strategic decision making regarding its active management. Further, it would give future review committees a better basis for their feedback. We further urge the MoF to investigate why NBIM does not take full advantage of its trackingerror limit. The objective of this study would be to understand why this ex-ante limit is not reached, whether any operational impediments or structural barriers play a role, and whether and how they can be lifted, if relevant.

- We suggest that NBIM improves the communication with external stakeholders on the Fund's active strategies and substrategies, giving access to more granular information on the internal benchmarking process and the cost allocation at the level of strategies, substrategies, and subsubstrategies. This may also involve providing more background on the active-risk appetite.

- We encourage the MoF to reduce the role of factor models in evaluating the Fund's performance and, instead, put more focus and emphasis on the qualitative assessments of the Fund's organization that investigate such modern aspects as governance quality, long-term mindset, and creating the appropriate culture for a successful execution of the strategy. We advise future 
review committees to evaluate NBIM's active management with simple, understandable, and widely accepted performance-evaluation models.

- If the MoF confirms the removal of real estate from NBIM's benchmark, we seriously doubt the long-term viability of NBIM managing unlisted-real-estate investments. If unlisted real estate is deemed important for diversification purposes by the MoF, we advise restoring real estate to the benchmark.

- We urge the MoF to provide clarity in the mandate on the objectives and prioritization of active ownership strategies, as well as on what parts of this prioritization are NBIM's purview versus which are prescribed in the mandate. 


\section{References}

Akepanidtaworn, K., Mascio, R. D., Imas, A. and Schmidt, L. (2021), Selling Fast and Buying Slow: Heuristics and Trading Performance of Institutional Investors, Working paper, National Bureau of Economic Research.

URL: http: //www.nber.org/papers/w29076

Akey, P., Robertson, A. and Simutin, M. (2021), Noisy Factors, Technical report, University of Toronto.

URL: https: //ssrn.com/abstract=3930228

Andonov, A., Bauer, R. and Cremers, M. (2012), Can Large Pension Funds Beat the Market? Asset Allocation, Market Timing, Security Selection, and the Limits of Liquidity, Technical report, Network for Studies on Pensions, Aging, and Retirement.

URL: https: //ssrn. com/abstract=2214931

Andonov, A., Eichholtz, P. and Kok, N. (2015), 'Intermediated Investment Management in Private Markets: Evidence from Pension Fund Investments in Real Estate', Journal of Financial Markets 22, 73-103.

Andonov, A., Kok, N. and Eichholtz, P. (2013), 'A Global Perspective on Pension Fund Investments in Real Estate', Journal of Portfolio Management 39(5), 32-42.

Ang, A. (2014), Asset Management: A Systematic Approach to Factor Investing, Oxford University Press.

Ang, A., Brandt, M. W. and Denison, D. F. (2014), Review of the Active Management of the Norwegian Government Pension Fund Global, Technical report, Norwegian Ministry of Finance.

URL: https://www.regjeringen. no/globalassets/upload/fin/statens-pensjonsfond/ eksterne-rapporter-og-brev/2014/angbrandtdenison_2014.pdf

Ang, A., Chen, L., Gates, M. and Henderson, P. D. (2021), 'Index + Factors + Alpha', Financial Analysts Journal pp. 1-20.

Ang, A., Goetzmann, W. N. and Schaefer, S. M. (2009), Evaluation of active management of the Norwegian Government Pension Fund Global, Technical report, Norwegian Ministry of Finance.

URL: https://www.regjeringen. no/globalassets/upload/fin/statens-pensjonsfond/ eksterne-rapporter-og-brev/ags-report.pdf

Antón, M., Cohen, R. B. and Polk, C. (2021), Best Ideas, Technical report, Social Science Research Network.

URL: https: //ssrn.com/abstract_id=1364827

Arnott, R., Harvey, C. R., Kalesnik, V. and Linnainmaa, J. (2019), 'Alice's Adventures in Factorland: Three Blunders that Plague Factor Investing', Journal of Portfolio Management 45(4), 18-36.

Bai, J., Bali, T. G. and Wen, Q. (2019), 'Common Risk Factors in the Cross-Section of Corporate Bond Returns', Journal of Financial Economics 131(3), 619-642.

Baltussen, G., Swinkels, L. and Van Vliet, P. (2021), 'Global Factor Premiums', Journal of Financial Economics 142(3), 1128-1154.

Barber, B. M. and Odean, T. (2013), The Behavior of Individual Investors, in 'Handbook of the Economics of Finance', Vol. 2, Elsevier, pp. 1533-1570.

Bartram, S. M., Lohre, H., Pope, P. F. and Ranganathan, A. (2021), 'Navigating the Factor Zoo around the World: An Institutional Investor Perspective', Journal of Business Economics pp. 1-49.

Berk, J. B. and Green, R. C. (2004), 'Mutual Fund Flows and Performance in Rational Markets', Journal of Political Economy 112(6), 1269-1295. 
Berk, J. B. and van Binsbergen, J. H. (2015), 'Measuring Skill in the Mutual Fund Industry', Journal of Financial Economics 118(1), 1-20.

Berk, J. B., Van Binsbergen, J. H. and Miller, M. (2020), 'Mutual Funds: Skill and Performance', Journal of Portfolio Management 46(5), 17-31.

Bessembinder, H. (2018), 'Do Stocks Outperform Treasury Bills?', Journal of Financial Economics 129(3), 440-457.

Bessembinder, H. (2021), 'Wealth Creation in the US Public Stock Markets 1926-2019', Journal of Investing 30(3), 47-61.

Black, F. (1986), 'Noise', Journal of Finance 41(3), 528-543.

Brinson, G. P., Hood, L. R. and Beebower, G. L. (1995), 'Determinants of Portfolio Performance', Financial Analysts Journal 51(1), 133-138.

Brooks, J., Palhares, D. and Richardson, S. (2018), 'Style Investing in Fixed Income', Journal of Portfolio Management 44(4), 127-139.

Busse, J. A., Goyal, A. and Wahal, S. (2010), 'Performance and Persistence in Institutional Investment Management', Journal of Finance 65(2), 765-790.

Carhart, M. M. (1997), 'On Persistence in Mutual Fund Performance', Journal of Finance 52(1), 57-82.

Carlo, A., Eichholtz, P. and Kok, N. (2021), 'Three Decades of Global Institutional Investment in Commercial Real Estate', Journal of Portfolio Management 47(10), 25-40.

CEM (2019), Investment Cost Effectiveness Analysis, Discussion note, Norwegian Ministry of Finance.

URL: https: // www. regjeringen. no/ contentassets/ c8dadbf763e44aaab1678f0f4c7b5226/ cem-ibs-2019-government-pension-fund-global.pdf

Chambers, D., Dimson, E. and Ilmanen, A. (2012), 'The Norway Model', Journal of Portfolio Management 38(2), 67-81.

Chambers, D., Dimson, E. and Ilmanen, A. (2021), 'The Norway model in perspective', Journal of Portfolio Management 47(5), 178-187.

Chen, H., Noronha, G. and Singal, V. (2006), 'Index Changes and Losses to Index Fund Investors', Financial Analysts Journal 62(4), 31-47.

Chen, Y., Ferson, W. and Peters, H. (2010), 'Measuring the Timing Ability and Performance of Bond Mutual Funds', Journal of Financial Economics 98(1), 72-89.

Cochrane, J. (2011), 'Presidential Address: Discount Rates', Journal of Finance 66(4), 1047-1108.

Cremers, K. M., Fulkerson, J. A. and Riley, T. B. (2019), 'Challenging the Conventional Wisdom on Active Management: A Review of the Past 20 years of Academic Literature on Actively Managed Mutual Funds', Financial Analysts Journal 75(4), 8-35.

Cremers, K. M. and Petajisto, A. (2009), 'How Active Is Your Fund Manager? A New Measure that Predicts Performance', Review of Financial Studies 22(9), 3329-3365.

Cremers, M. and Pareek, A. (2016), 'Patient Capital Outperformance: The Investment Skill of High Active Share Managers Who Trade Infrequently', Journal of Financial Economics 122(2), 288-306.

Dahlquist, M. and Ødegaard, B. A. (2018), A Review of Norges Bank's Active Management of the Government Pension Fund Global, Technical report, Norwegian Ministry of Finance.

URL: https: //www. regjeringen. no/ contentassets/7fb88d9696a34ea6a0cd9225628711a9/ review_dahlquistodegaard_2018.pdf 
Dahlquist, M., Polk, C., Priestley, R. and Ødegaard, B. A. (2015), Norges Bank's Expert Group on Principles for Risk Adjustment of Performance Figures - Final Report, Technical report, Norges Bank Investment Management.

URL: https: // www. nbim. no/ contentassets/f04b97db0 e704572bfbda10525a6a3fc/ expert_ group_final_report_nov_2015. pdf

Daniel, K., Grinblatt, M., Titman, S. and Wermers, R. (1997), 'Measuring Mutual Fund Performance with Characteristic-Based Benchmarks', Journal of Finance 52(3), 1035-1058.

Dellva, W. L., DeMaskey, A. L. and Smith, C. A. (2001), 'Selectivity and Market Timing Performance of Fidelity Sector Mutual Funds', Financial Review 36(1), 39-54.

Dimson, E., Karakaş, O. and Li, X. (2015), 'Active Ownership', Review of Financial Studies 28(12), 3225-3268.

Dimson, E., Karakaş, O. and Li, X. (2021), Coordinated Engagements, Technical report, Social Science Research Network.

URL: https: //ssrn. com/abstract_ $i d=3209072$

Dyakov, T., Jiang, H. and Verbeek, M. (2019), 'Trade Less and Exit Overcrowded Markets: Lessons from International Mutual Funds*', Review of Finance 24(3), 677-731.

Dyck, A., Lins, K. V. and Pomorski, L. (2013), 'Does Active Management Pay? New International Evidence', Review of Asset Pricing Studies 3(2), 200-228.

Døskeland, T. M. and Stromberg (2018), Evaluating Investments in Unlisted Equity for the Norwegian Government Pension Fund Global (GPFG), Technical report, Norwegian Ministry of Finance.

URL: https: // www. regjeringen. no/ contentassets/7fb88d969ba34ea6a0cd9225b28711a9/ evaluating_doskelandstromberg_05042018.pdf

Elton, E. J. and Gruber, M. J. (2020), 'A Review of the Performance Measurement of Long-Term Mutual Funds', Financial Analysts Journal 76(3), 22-37.

Elton, E. J., Gruber, M. J. and Souza, A. d. (2021), 'Are Enhanced Index Funds Enhanced?', European Financial Management (Advanced online publication).

Fama, E. F. (1970), 'Efficient Capital Markets: A Review of Theory and Empirical Work', Journal of Finance 25(2), 383-417.

Fama, E. F. and French, K. R. (1993), 'Common Risk Factors in the Returns on Stocks and Bonds', Journal of Financial Economics 33(1), 3-56.

Fama, E. F. and French, K. R. (2010), 'Luck versus Skill in the Cross-Section of Mutual Fund Returns', Journal of Finance 65(5), 1915-1947.

Fama, E. F. and French, K. R. (2015), 'A Five-Factor Asset Pricing Model', Journal of Financial Economics 116(1), 1-22.

Fama, E. and French, K. (1992), 'The Cross-section of Expected Stock Returns', Journal of Finance 47(2), 427-465.

Feng, G., Giglio, S. and Xiu, D. (2020), 'Taming the Factor Zoo: A Test of New Factors', Journal of Finance 75(3), 1327-1370.

Ferson, W. E. and Schadt, R. W. (1996), 'Measuring Fund Strategy and Performance in Changing Economic Conditions', Journal of Finance 51(2), 425-461.

Frazzini, A. and Pedersen, L. H. (2014), 'Betting against Beta', Journal of Financial Economics 111(1), 1-25. 
French, K. R. (2008), 'Presidential Address: The Cost of Active Investing', Journal of Finance 63(4), 1537-1573.

Fulkerson, J. A. (2013), 'Is Timing Everything? The Value of Mutual Fund Manager Trades', Financial Management 42(2), 243-261.

Gârleanu, N. and Pedersen, L. H. (2018), 'Efficiently Inefficient Markets for Assets and Asset Management', Journal of Finance 73(4), 1663-1712.

Gerakos, J., Linnainmaa, J. T. and Morse, A. (2021), 'Asset Managers: Institutional Performance and Factor Exposures', Journal of Finance 76(4), 2035-2075.

Goetzmann, W. N., Spaenjers, C. and van Nieuwerburgh, S. (2021), 'Real and Private-Value Assets'. URL: http: //www. nber. org/papers/w28580

Goodwin, T. H. (1998), 'The Information Ratio', Financial Analysts Journal 54(4), 34-43.

Goyal, A. and Wahal, S. (2008), 'The Selection and Termination of Investment Management Firms by Plan Sponsors', Journal of Finance 63(4), 1805-1847.

Grinold, R. C. (1989), 'The Fundamental Law of Active Management', Journal of Portfolio Management 15(3), 30-37.

Grinold, R. C. and Kahn, R. N. (2000), Active Portfolio Management, McGraw Hill New York.

Grossman, S. J. and Stiglitz, J. E. (1980), 'On the Impossibility of Informationally Efficient Markets', American Economic Review 70(3), 393-408.

Hamaui, A. and Jaffard, P. (2021), Chasing the Beta, Losing the Alpha, Technical report, Social Science Research Network.

URL: https: //ssrn. com/abstract_ $i d=3816944$

Harvey, C. R. and Liu, Y. (2020), 'False (and Missed) Discoveries in Financial Economics', Journal of Finance 75(5), 2503-2553.

Henriksson, R. D. and Merton, R. C. (1981), 'On Market Timing and Investment Performance. II. Statistical Procedures for Evaluating Forecasting Skills', Journal of Business pp. 513-533.

Hou, K., Mo, H., Xue, C. and Zhang, L. (2019), 'Which Factors?', Review of Finance 23(1), 1-35.

Jenkinson, T., Jones, H. and Martinez, J. V. (2016), 'Picking Winners? Investment Consultants' Recommendations of Fund Managers', Journal of Finance 71(5), 2333-2370.

Jensen, M. C. (1968), 'The Performance of Mutual Funds in the Period 1945-1964', Journal of Finance $\mathbf{2 3}(2), 389-416$.

Kacperczyk, M., Nieuwerburgh, S. V. and Veldkamp, L. (2014), 'Time-Varying Fund Manager Skill', Journal of Finance 69(4), 1455-1484.

Lewellen, J. (2011), 'Institutional Investors and the Limits of Arbitrage', Journal of Financial Economics 102(1), 62-80.

Li, F., Chow, T.-M., Pickard, A. and Garg, Y. (2019), 'Transaction Costs of Factor-Investing Strategies', Financial Analysts Journal 75(2), 62-78.

Lo, A. W. (2002), 'The Statistics of Sharpe ratios', Financial Analysts Journal 58(4), 36-52.

Markowitz, H. (1952), 'Portfolio Selection', Journal of Finance 7, 77-91. 
Ministry of Finance (2021), GPFG's Investment Mandate, Technical report, Norwegian Ministry of Finance.

URL: https: //www. regjeringen. no/ contentassets/9d68c55c272c41e99f0bf45d24397d8c/ gpfg_mandate_2021.08.24.pdf

Minstry of Finance (2021), Evaluation of Climate Risk, Technical report, Norwegian Ministry of Finance.

URL: https: // www. regjeringen. no/ contentassets/05c6e37b84624545a3aaf4276cd79acb/ 2021-03-05-brev-spu.pdf

Moneta, F. (2015), 'Measuring Bond Mutual Fund Performance with Portfolio Characteristics', Journal of Empirical Finance 33, 223-242.

Natter, M., Rohleder, M. and Wilkens, M. (2021), 'Maturity-Matched Bond Fund Performance', Financial Analysts Journal 77(2), 83-96.

NBIM (2014), Benchmark Design for an Active Investment Process, Discussion note, Norges Bank Investment Management.

URL: $\quad$ https://www.nbim.no/globalassets/documents/dicussion-paper/2014/nbim_ discussionnotes_6-14.pdf/

NBIM (2017), Review of Norges Bank's management of the Government Pension Fund Global, Letter to Ministry of Finance, Norges Bank Investment Management.

URL: Ittps://www.nbim.no/en/publications/submissions-to-ministry/2017/ review-of-norges-banks-management-of-the-government-pension-fund-global/

NBIM (2020a), Factor and Risk-Adjusted Return 2020, Technical report, Norges Bank Investment Management.

URL: $\quad h t t p s: / / w w w . n b i m . n o / c o n t e n t a s s e t s / f d 871 d 2 a 4 e 2 d 4 c 1 a b 9 d 3 d 66 c 98 f a 6 b a 1 /$ factor-and-risk-adjusted-return. pdf

NBIM (2020b), Government Pension Fund Global Annual Report 2020, Technical report, Norges Bank Investment Management.

URL: $\quad h t t p s: / / w w w . n b i m . n o /$ contentassets/fd871d2a4e2d4c1ab9d3d66c98fabba1/ annual-report_2020_government-pension-fund-global_web

NBIM (2020c), Investing in Equities, Technical report, Norges Bank Investment Management.

URL: $\quad h t t p s: / / w w w . n b i m . n o / c o n t e n t a s s e t s / 3 c 31 b f 0882 e 445 b 38$ ef45a1377cc0513/ investing-in-equities_government-pension-fund-global_web_final.pdf

NBIM (2020d), Investing in Real Estate, Technical report, Norges Bank Investment Management.

URL: https://www.nbim.no/contentassets/5e6fa39689564c33654935780416172d/ investing-in-real-estate_the-10-year-history.pdf

NBIM (2020e), Investing Responsibly, Technical report, Norges Bank Investment Management.

URL: https: //www. nbim.no/contentassets/aee68d3bc8e145c8bc5c5636c1bafe5b/ investing-responsibly_government-pension-fund-global_web. pdf

NBIM (2020f), Investing with a Mandate, Technical report, Norges Bank Investment Management.

URL: $\quad h t t p s: / / w w w . n b i m . n o / c o n t e n t a s s e t s / c d 563 b 586 f e 34 c e 2 b f e a 30 d f 4 c 0 a 75 d b /$ investing-with-a-mandate_government-pension-fund-global_web. pdf

NBIM (2020g), Investing with Company Insight, Technical report, Norges Bank Investment Management.

URL: $\quad h t t p s: / / w w w . n b i m . n o / c o n t e n t a s s e t s / a a 58 b 0677 f e 54 a b 4 a c c d d 36 a d 48154 b 3 /$ investing-with-company-insight_web. pdf 
NBIM (2020h), Investing with External Managers, Technical report, Norges Bank Investment Management.

URL: $\quad h t t p s: / / w w w . n b i m . n o / c o n t e n t a s s e t s / 62 a 6 c 07 f b 01641$ e3a6ab7fe27f23de69/ investing-with-external-managers_the-20-year-history.pdf

NBIM (2020i), NBIM Performance Results GIPS Report 2020, Technical report, Norges Bank Investment Management.

URL: $\quad$ https: // www. nbim.no/ contentassets/98750345a7e641558d820573c52e4a8e/ gips-report-2020.pdf

NBIM (2020j), Responsible Investment 2020, Technical report, Norges Bank Investment Management. URL: $\quad h t t p s: / / w w w . n b i m . n o /$ contentassets/fefoe2802b3f423ba2e514cfde127rdr/ government-pension-fund-global_2020_responsible-investment_web. pdf

NBIM (2021a), Climate Risk in the Government Pension Fund Global, Letter to Ministry of Finance, Norges Bank Investment Management.

URL: Ittps://www.nbim.no/en/publications/submissions-to-ministry/2020/ climate-risk-in-the-government-pension-fund-global/

NBIM (2021b), Government Pension Fund Global - Experience of the Climate Criterion, Letter to Ministry of Finance, Norges Bank Investment Management.

URL: $\quad h t t p s: / / w w w . n b i m . n o /$ contentassets/3fdc0db6dee04ddba6c5\%c17d5ac3adb/ government-pension-fund-global--experience-of-the-climate-criterion.pdf

NBIM (2021c), Investing in Fixed Income, Technical report, Norges Bank Investment Management.

URL: $\quad h t t p s: / / w w w . n b i m . n o /$ contentassets/721089d167ba4b6597f099cdc761673f/ investing-in-fixed-income_web. pdf

NBIM (2021d), Review of the management of the Government Pension Fund Global, Letter to Ministry of Finance, Norges Bank Investment Management.

URL: $\quad h t t p s: / / w w w . n b i m . n o /$ contentassets/d624c8017f2c4c2787b699e80db3c8a5/ review-of-the-management-of-the-government-pension-fund-global.pdf

NBIM (2021e), Strategy 2021-2022, Technical report, Norges Bank Investment Management.

URL: $\quad h t t p s: / / w w w . n b i m . n o / c o n t e n t a s s e t s / 579978 c 29 f b a 46839 d 505 b e 5 c 5 f 19950 /$ nbim-strategy-2021_2022_eng_web. pdf

NBIM (2021f), Work on Climate Risk in the Government Pension Fund Global, Letter to Ministry of Finance, Norges Bank Investment Management.

URL: $\quad h t t p s: / / w w w . n b i m . n o / c o n t e n t a s s e t s / c 4353362 c 1904 b 8800387 e 2485 a e 4681 /$ work-on-climate-risk-in-the-government-pension-fund-global.pdf

Newey, W. K. and West, K. D. (1987), 'A Simple, Positive Semi-Definite, Heteroskedasticity and Autocorrelation Consistent Covariance Matrix', Econometrica 55(3), 703-708.

Novy-Marx, R. and Velikov, M. (2016), 'A Taxonomy of Anomalies and Their Trading Costs', Review of Financial Studies 29(1), 104-147.

Pástor, L., Stambaugh, R. F. and Taylor, L. A. (2015), 'Scale and Skill in Active Management', Journal of Financial Economics 116(1), 23-45.

Pastor, L., Stambaugh, R. F. and Taylor, L. A. (2021a), Dissecting Green Returns, Technical report, National Bureau of Economic Research.

URL: http: // www. nber. org/papers/w28940

Pástor, L., Stambaugh, R. F. and Taylor, L. A. (2021b), 'Sustainable Investing in Equilibrium', Journal of Financial Economics 142(2), 550-571.

Pedersen, L. H. (2015), Efficiently Inefficient, Princeton University Press. 
Pedersen, L. H. (2018), 'Sharpening the Arithmetic of Active Management', Financial Analysts Journal 74(1), 21-36.

Sensoy, B. A. (2009), 'Performance Evaluation and Self-Designated Benchmark Indexes in the Mutual Fund Industry', Journal of Financial Economics 92(1), 25-39.

Sharpe, W. F. (1981), 'Decentralized Investment Management', Journal of Finance 36(2), 217-234.

Sharpe, W. F. (1991), 'The Arithmetic of Active Management', Financial Analysts Journal 47(1), 7-9.

Sharpe, W. F. (1992), 'Asset Allocation: Management Style and Performance Measurement', Journal of Portfolio Management 18(2), 7-19.

Skancke, M., Halvorsen, K., Hanstad, T. B. and Thornburn, K. (2021), Climate Risk and the Government Pension Fund Global, Technical report, Norwegian Ministry of Finance.

URL: https: // www. regjeringen. no/ contentassets/ fb49a0e957324d7caadb625c6ec4490c/ en-gb/pdfs/r-0655-e-klimarisiko-og-oljefondet.pdf

Tinbergen, J. (1952), On the Theory of Economic Policy, North-Holland Publishing Company, Amsterdam.

Treynor, J. L. and Black, F. (1973), 'How to Use Security Analysis to Improve Portfolio Selection', Journal of Business 46(1), 66-86.

Treynor, J. and Mazuy, K. (1966), 'Can Mutual Funds Outguess the Market', Harvard Business Review 44(4), 131-136.

van Binsbergen, J. H., Brandt, M. W. and Koijen, R. S. J. (2008), 'Optimal Decentralized Investment Management', Journal of Finance 63(4), 1849-1895.

van Binsbergen, J. H., Han, J., Ruan, H. and Xing, R. (2021), An Equilibrium Model of Career Concerns, Investment Horizons, and Mutual Fund Value Added, Technical report, Social Science Research Network.

URL: https: //ssrn. com/abstract_id=3920863

van Binsbergen, J. H. and Koijen, R. (2018), Benchmarking Global Fixed Income Portfolios, Technical report, Norwegian Ministry of Finance.

URL: https: //www. regjeringen. no/ contentassets/7fb88d969ba34ea6a0cd9225628711a9/ 2018_benchmarking_fixed_income_gpfg.pdf

van Nieuwerburgh, S., Stanton, R. and de Bever, L. (2015), A Review of Real Estate and Infrastructure Investments by the Norwegian Government Pension Fund Global (GPFG), Technical report, Norwegian Ministry of Finance.

URL: https: // www. regjeringen. no/ contentassets/f353169233704a55b3af6b0b36fb3129/ ekspertrapport_eiendom_infrastruktur. pdf

Wasserstein, R. L. and Lazar, N. A. (2016), 'The ASA Statement on $p$-Values: Context, Process, and Purpose', American Statistician 70(2), 129-133.

Wermers, R. (2000), 'Mutual Fund Performance: An Empirical Decomposition into Stock-Picking Talent, Style, Transactions Costs, and Expenses', Journal of Finance 55(4), 1655-1695.

Wermers, R. (2011), 'Performance Measurement of Mutual Funds, Hedge Funds, and Institutional Accounts', Annual Review of Financial Economics 3(1), 537-574.

Wermers, R. (2021), 'Active Investing and the Efficiency of Security Markets', Journal of Investment Management 19(1).

URL: https: // joim.com/downloads/active-investing-and-the-efficiency-of-security- I markets/ 


\section{Appendices}

\section{A Mandate}

Mandate for the expert group reviewing Norges Bank's active management of the GPFG

The expert group shall, by 3 January 2022, prepare a public report reviewing Norges Bank's active management of the Government Pension Fund Global (GPFG). The report shall include the following:

1. A review of Norges Bank's historical financial performance in the management of the GPFG, including:

- Analysis of risk and return relative to relevant benchmarks for the Fund overall and for the equity and fixed-income portfolio, respectively.

- Performance and attribution analysis of Norges Bank's main investment strategies, including significant substrategies.

- Analysis of risk-adjusted performance of the above-mentioned portfolios and strategies, including a discussion of the appropriate choice of methodology for risk-adjustment and relevant risk factors for a fund with GPFG's characteristics. Recognised, leading methods should be applied.

2. An assessment of the results from Norges Bank's real-estate strategy, including:

- Analysis of the real-estate portfolio's contribution to the Fund's risk and return since 2017. The analysis should consider both the total real-estate portfolio and respective listed and unlisted portfolios. It should also include the effect from Norges Bank's chosen financing model.

3. A qualitative assessment of Norges Bank's current main investment strategies and significant substrategies in the management of the GPFG. The assessment shall include discussions of

- theoretical and empirical foundations,

- known risk and return characteristics,

- appropriate evaluation horizons,

- scalability.

4. An assessment of Norges Bank's potential to obtain excess returns in the management of the GPFG relative to the current strategic benchmark, given the Fund distinctive characteristics as large, state owned and with a long investment horizon. 


\section{B Data}

\section{Returns and AUM for the Fund}

Some of the data series for the Fund are available at NBIM's homepage. Others we obtained directly from NBIM.

For returns at the highest portfolio levels, see https://www.nbim.no/en/publications/reports/ 2021/half-year-report-2021/. However, the latest three months are not available there and come to us directly from NBIM.

The returns for the main investment strategies are available at https://www.nbim.no/en/publications/ reports/2020/annual-report-2020/ but not for every substrategy. The data available online covers only to 2020. The strategies not available online and the updated data series come to us directly from NBIM.

For details about how NBIM calculates returns, see https://www.nbim.no/en/the-fund/returns/ methodology-for-the-calculation-of-returns/ and the latest GICS report (NBIM, 2020i).

AUM information is not publicly available for any of the Fund's strategies and so comes directly from NBIM.

\section{Factor variables}

As a base case, we use the seven-factor model with the following factors:

- MKT: Market, the return on a world market portfolio minus the US one-month T-bill rate

- $S M B$ : Small minus big, the return on a small stock portfolio minus the return on a big stock portfolio.

- $H M L$ : High minus low, the return on a value portfolio minus the return on a growth portfolio.

- $R M W$ : Robust minus weak, the return on a robust-operating-profitability portfolio minus the return on a weak-operating-profitability portfolio. Often labeled a quality factor.

- CMA: Conservative minus aggressive, the return on a conservative investment portfolio minus the return on an aggressive investment portfolio. Also, often labeled a quality factor.

- TERM: Term premium, the return on a global index with Treasuries maturing in more than ten years minus the return on a global Treasury index maturing in one to three years.

- DEF : Duration adjusted default premium, the return on a portfolio of corporate bonds minus the return on a portfolio of Treasury bonds. The maturities are over ten years and adjusted for different duration.

\section{Factors from Kenneth French' data library}

We obtained five factors, $M K T, S M B, H M L, R M W$, and $C M A$, on November 7, 2021, from Kenneth French' data library: http://mba.tuck.dartmouth. edu/pages/faculty/ken.french/Data Library/f-f_5developed.html. As shown in Akey et al. (2021), these factors change with factor vintage.

\section{Factors from NBIM}

We obtained two factors from NBIM, TERM and DEF. These factors are used and further described in NBIM's Factor and risk-adjusted report (NBIM, 2020a).

\section{Currency conversion}

Dahlquist and Ødegaard (2018) report their results in the Fund's currency basket (CB), while NBIM (2020a) reports their results in USD. CB is a weighted basket of currencies in the Fund's benchmark. Since NBIM delivers return data in both $R_{t}^{\mathrm{CB}}$ and $R_{t}^{\mathrm{USD}}$, we "back out" the currency depreciation rate at time $t\left(X_{t}\right)$ using the following identity.

$$
1+X_{t}=\frac{1+R_{t}^{C B}}{1+R_{t}^{U S D}}
$$




\section{Appendix for Section 2}

\section{Valid benchmark properties}

The properties of a valid benchmark (see Section 2.2):

- Unambiguous: The names and weights of component securities should be known (this rules out unknown derived benchmarks, such as those based on the arbitrage-pricing-theory factors).

- Tradable: It should be available as a passive investment alternative for the manager.

- Measurable: It must be possible to compute a valid return on the benchmark periodically. This might not be possible for benchmarks with illiquid assets.

- Appropriate: The benchmark must reflect the manager's style.

- Reflective of current investment opinions: A manager should be able to form an opinion on the expected rate of return on the benchmark.

- Specified in advance: It should give the manager a passive alternative ahead of time, to make clear the measuring tape. (Wermers, 2011)

\section{Additional figures and tables: Fund portfolio}

- Figure 25

- Table 39

- Figure 26

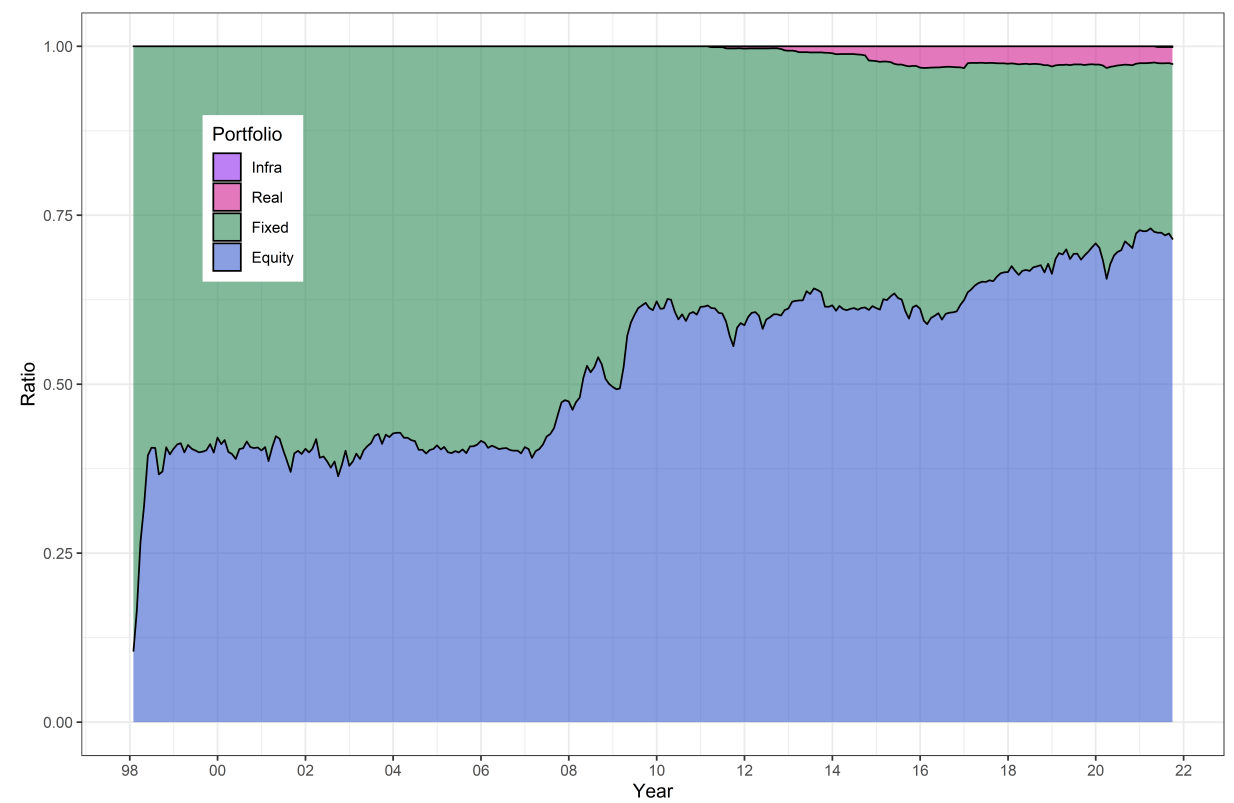

Figure 25: Fund asset allocation 


\begin{tabular}{|c|c|c|c|c|c|c|c|c|c|}
\hline \multirow[b]{2}{*}{ Return } & \multicolumn{3}{|c|}{ Fund portfolio } & \multicolumn{3}{|c|}{ Equity portfolio } & \multicolumn{3}{|c|}{ Fixed-income portfolio } \\
\hline & Gross & Net & Bench & Gross & Net & Bench & Gross & Net & Bench \\
\hline & \multicolumn{9}{|c|}{ Full sample (1998-Sep 2021) } \\
\hline Geo. mean & 6.31 & 6.23 & 6.06 & 6.90 & 6.79 & 6.51 & 4.48 & 4.43 & 4.32 \\
\hline Arit. mean & 6.63 & 6.55 & 6.35 & 8.00 & 7.89 & 7.57 & 4.53 & 4.49 & 4.37 \\
\hline Std. dev. & 8.00 & 8.00 & 7.68 & 14.71 & 14.71 & 14.43 & 3.33 & 3.33 & 3.22 \\
\hline Sharpe ratio & 0.62 & 0.61 & 0.61 & 0.43 & 0.42 & 0.41 & 0.86 & 0.84 & 0.83 \\
\hline \multirow[t]{2}{*}{$N$} & 285 & 285 & 285 & 284 & 284 & 284 & 285 & 285 & 285 \\
\hline & \multicolumn{9}{|c|}{ Latest sample (2017-Sep 2021) } \\
\hline Geo. mean & 9.31 & 9.26 & 9.05 & 11.67 & 11.61 & 11.45 & 3.44 & 3.41 & 3.18 \\
\hline Arit. mean & 9.78 & 9.73 & 9.51 & 12.64 & 12.58 & 12.39 & 3.49 & 3.46 & 3.23 \\
\hline Std. dev. & 9.75 & 9.75 & 9.66 & 13.92 & 13.92 & 13.74 & 3.12 & 3.12 & 3.17 \\
\hline Sharpe ratio & 0.97 & 0.96 & 0.95 & 0.88 & 0.88 & 0.88 & 1.01 & 1.00 & 0.91 \\
\hline$N$ & 57 & 57 & 57 & 57 & 57 & 57 & 57 & 57 & 57 \\
\hline
\end{tabular}

Notes: The table shows statistics for the gross, net and benchmark returns for the total Fund portfolio, the equity portfolio, and the fixed-income portfolio. The table shows the annualized geometric mean, arithmetic mean, standard deviation, Sharpe ratio, and number of observations, $N$. All returns are expressed as percentages of the Fund's currency basket.

Table 39: Total returns and risk (Fund's currency basket)

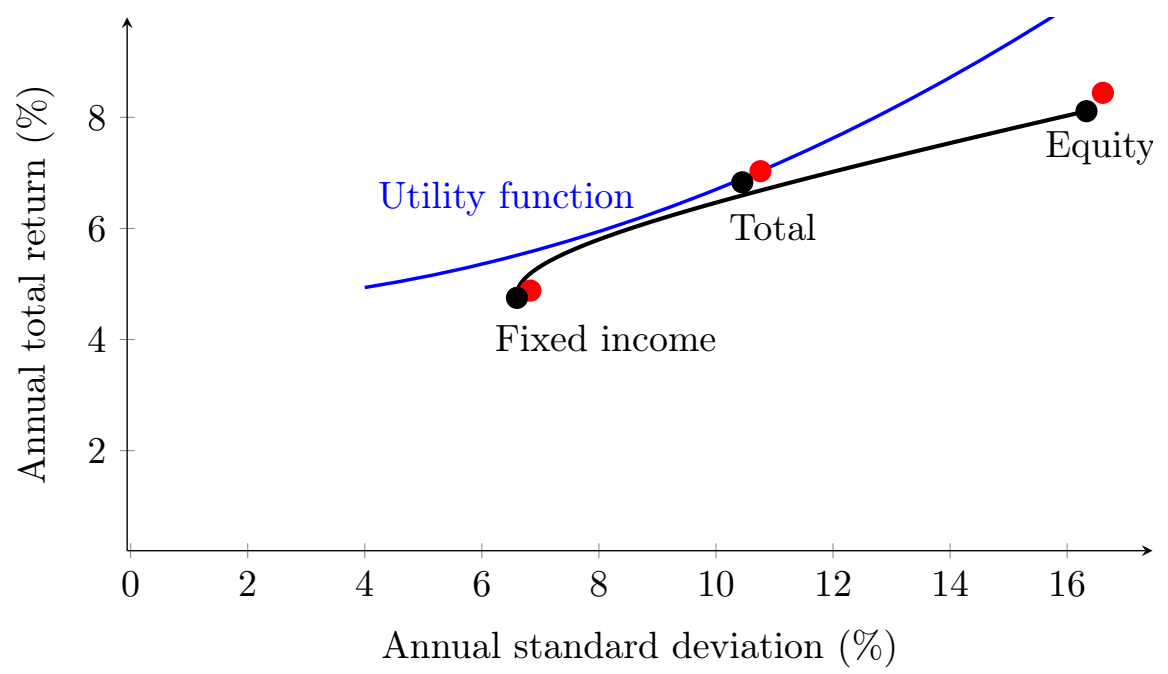

Total return and risk for Fund portfolios after cost (red dots) and benchmark portfolios (black dots). Sample period: Jan 1998-Sep 2021.

Figure 26: Total return-risk space 


\section{Appendix for Section 3}

\section{Additional tables}

- Table 40

- Table 41

- Table 42

\begin{tabular}{lcc|cc}
\hline & \multicolumn{2}{c|}{ Full sample (1998-Sep 2021) } & \multicolumn{2}{c}{ Latest sample (2017-Sep 2021) } \\
\cline { 2 - 5 } & Return & VA & Return & VA \\
\hline Gross & 0.27 & 0.78 & 0.27 & 2.28 \\
Costs & 0.08 & 0.20 & 0.05 & 0.41 \\
Net & 0.19 & 0.58 & 0.22 & 1.87 \\
\hline
\end{tabular}

Notes: The table shows the costs and the gross and net mean annualized active returns and value added. Returns expressed in percent and value added in NOK billion. Returns in the Fund's currency basket.

Table 40: Fund realized mean active returns (Fund's currency basket)

\begin{tabular}{lcc|cc}
\hline & \multicolumn{2}{c|}{ Full sample $(1998-$ Sep 2021) } & \multicolumn{2}{c}{ Latest sample $(2017-$ Sep 2021) } \\
\cline { 2 - 5 } & Gross & Net & Gross & Net \\
\hline Mean & 0.27 & 0.19 & $0.27^{*}$ & 0.22 \\
& $(0.23)$ & $(0.22)$ & $(0.14)$ & $(0.14)$ \\
$p$-value & .23 & .39 & .06 & .13 \\
$N$ & 285 & 285 & 57 & 57 \\
\hline
\end{tabular}

Notes: The table shows the mean annualized active return percent. Newey and West (1987) standard errors in parentheses. $N$ refers to the number of observations in the period. We denote by stars if the mean is significantly different from zero. ${ }^{* * * / * * / *}$ indicates significance at the $1 \% / 5 \% / 10 \%$ level.

Table 41: Fund mean active returns (Fund's currency basket)

\begin{tabular}{lcc|cc}
\hline & \multicolumn{2}{c|}{ Full sample (1998-Sep 2021) } & \multicolumn{2}{c}{ Latest sample (2017-Sep 2021) } \\
\cline { 2 - 5 } & Gross return & Net return & Gross return & Net return \\
\hline$\alpha$ & 0.14 & 0.06 & 0.19 & 0.14 \\
& $(0.21)$ & $(0.21)$ & $(0.13)$ & $(0.13)$ \\
$p$-value & .51 & .78 & .13 & .27 \\
$\beta$ & $1.03^{* * *}$ & $1.03^{* * *}$ & $1.01^{* * *}$ & $1.01^{* * *}$ \\
& $(0.01)$ & $(0.01)$ & $(0.003)$ & $(0.003)$ \\
$p$-value & .01 & .01 & .01 & .01 \\
$N$ & 285 & 285 & 57 & 57 \\
$R^{2}$ & 1.00 & 1.00 & 1.00 & 1.00 \\
\hline
\end{tabular}

Notes: The table shows the estimated $\alpha$ and $\beta$ from Eq. (1) for the gross and net returns in the full and latest sample periods. Returns expressed in percentage terms. Newey and West (1987) standard errors in parentheses. $N$ is the number of observations in the period, and $R^{2}$ is the explanatory power from the regression. We denote by stars if $\alpha$ is significantly different from zero and if $\beta$ is significantly different from one. ${ }^{* * * / * * / *}$ indicates significance at the 1\%/5\%/10\% level.

Table 42: Fund beta-adjusted active returns (Fund's currency basket) 


\section{E Appendix for Section 4}

\section{Additional figures and tables: Equity portfolio}

- Table 43

- Figure 27

- Figure 28

\begin{tabular}{lcc|cc}
\hline & \multicolumn{2}{c|}{ Full sample (1998-Sep 2021) } & \multicolumn{2}{c}{ Latest sample (2017-Sep 2021) } \\
\cline { 2 - 5 } & Gross return & Net return & Gross return & Net return \\
\hline Mean & 0.47 & 0.36 & 0.40 & 0.34 \\
Std. dev. & 0.73 & 0.73 & 0.37 & 0.37 \\
IR & 0.64 & 0.49 & 0.64 & 0.49 \\
Hit ratio & 0.65 & 0.63 & 0.72 & 0.70 \\
AC(1) & 0.17 & 0.17 & 0.06 & 0.06 \\
Skewness & -0.65 & -0.69 & -0.58 & -0.57 \\
Exc. kurtosis & 9.23 & 9.31 & -0.11 & -0.12 \\
$N$ & 285 & 285 & 57 & 57 \\
\hline
\end{tabular}

Notes: The table shows descriptive statistics for the gross annualized active returns in percent. IR refers to the mean divided by the standard deviation. Hit ratio is the fraction of months with a positive outcome. $\mathrm{AC}(1)$ refers to the first-order autocorrelation. $N$ is the number of observations in the period.

Table 43: Equity summary statistics of active returns

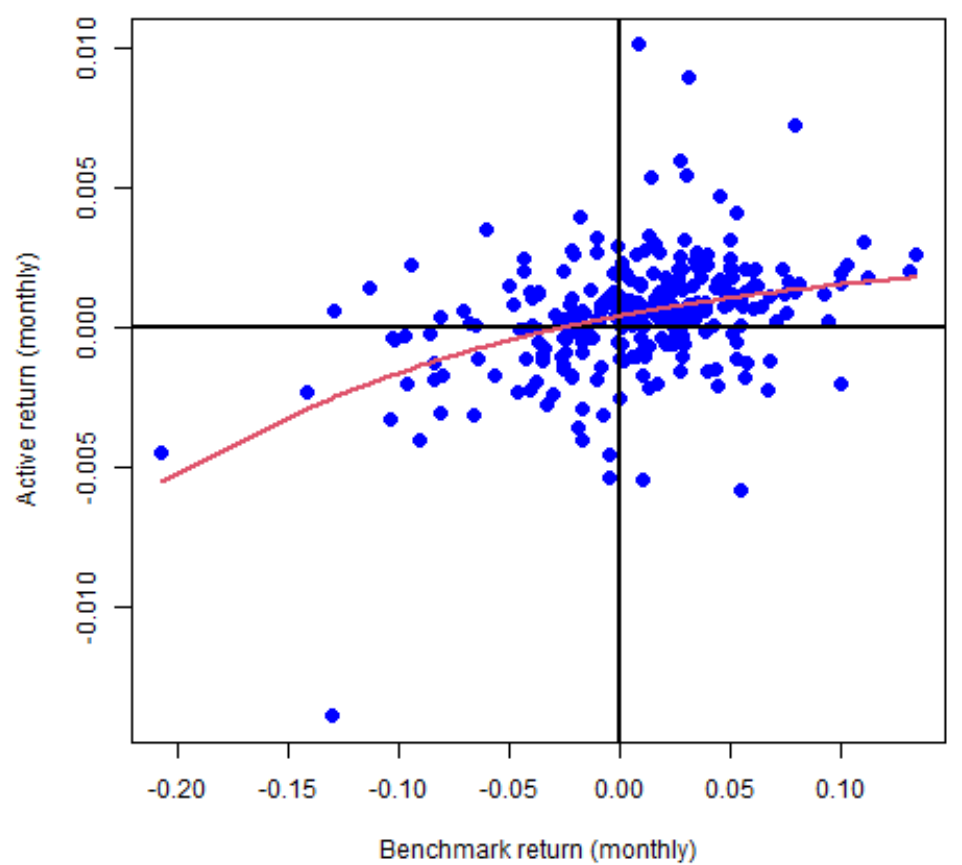

Figure 27: Equity active returns against benchmark returns 


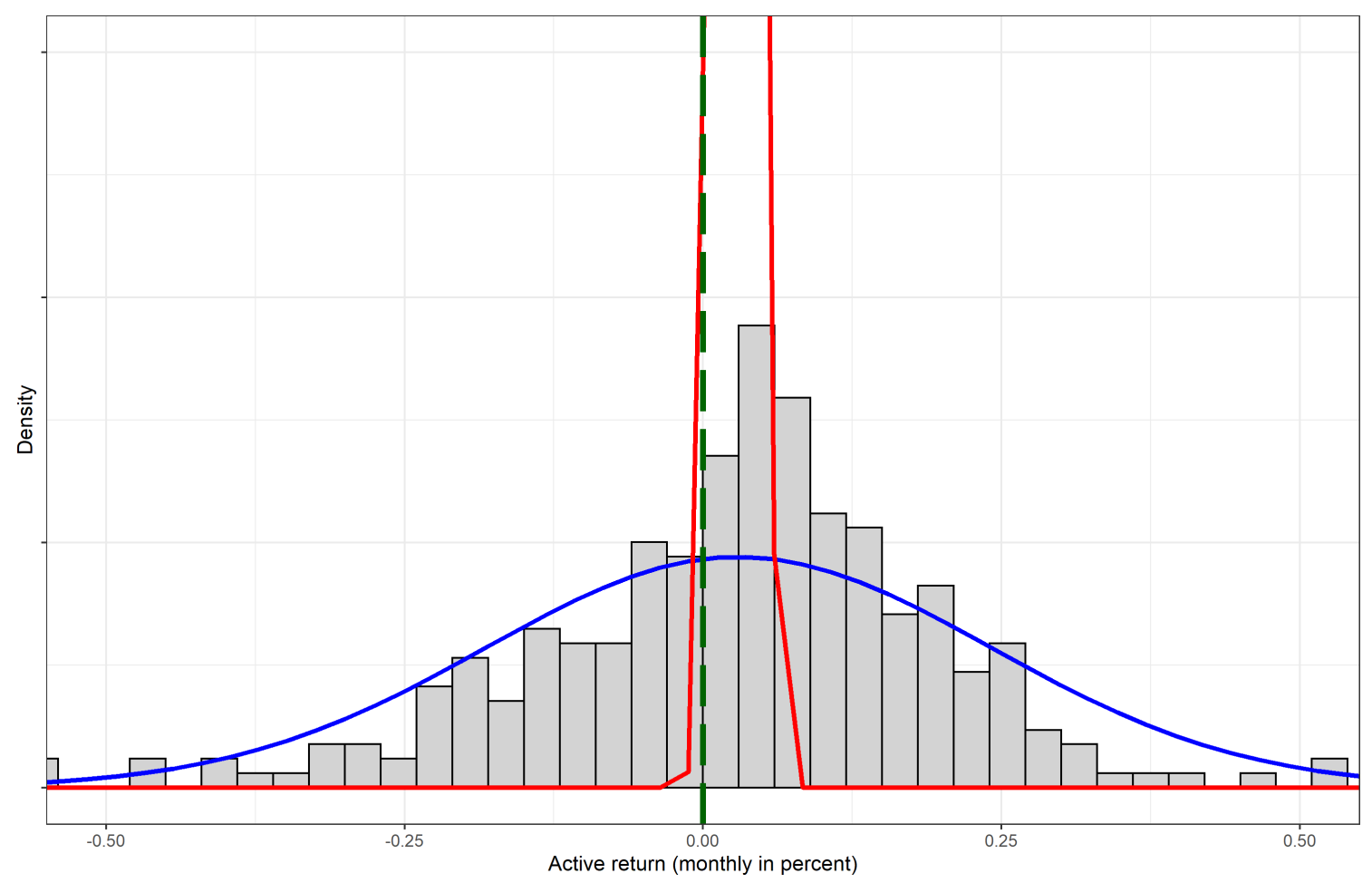

Figure 28: Histogram of the equity net active returns 


\section{F Appendix for Section 5}

\section{Additional tables: Equity subportfolios}

- Table 44

- Table 45

\begin{tabular}{|c|c|c|c|c|c|c|c|c|c|c|c|}
\hline Return & Equity & $\begin{array}{l}\text { Security } \\
\text { selection }\end{array}$ & Internal & External & Enhanced & Position & Lending & $\begin{array}{c}\text { Fund } \\
\text { allocation }\end{array}$ & Factors & Environment & Allocation \\
\hline \multicolumn{12}{|c|}{ 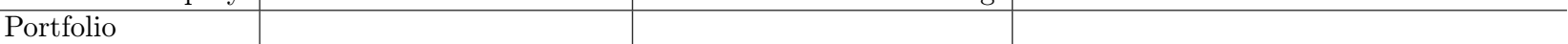 } \\
\hline Mean & 11.05 & 9.87 & 10.70 & 8.04 & 11.23 & 11.22 & 10.75 & 10.63 & 10.61 & 14.82 & 10.63 \\
\hline Std. dev. & 13.79 & 15.83 & 16.31 & 16.07 & 13.44 & 13.43 & 13.59 & 13.69 & 13.67 & 15.01 & 13.62 \\
\hline Sharpe & 0.76 & 0.58 & 0.62 & 0.46 & 0.79 & 0.79 & 0.75 & 0.73 & 0.73 & 0.95 & 0.74 \\
\hline \multicolumn{12}{|c|}{ Benchmark } \\
\hline Mean & 10.68 & 8.45 & 9.72 & 5.44 & 11.04 & 11.09 & 10.68 & 10.68 & 10.65 & 10.97 & 10.68 \\
\hline Std. dev. & 13.59 & 15.21 & 15.50 & 15.97 & 13.45 & 13.43 & 13.59 & 13.59 & 13.61 & 13.85 & 13.59 \\
\hline Sharpe & 0.77 & 0.51 & 0.59 & 0.30 & 0.77 & 0.78 & 0.74 & 0.74 & 0.74 & 0.75 & 0.74 \\
\hline$N$ & 105 & 105 & 105 & 105 & 105 & 105 & 105 & 105 & 105 & 105 & 105 \\
\hline
\end{tabular}

Notes: The table shows the mean and standard deviation of the annualized absolute returns and the Sharpe ratio. Returns expresed in percent for USD.

Table 44: Equity-strategies total return and risk

\begin{tabular}{|c|c|c|c|c|c|c|c|c|c|c|c|}
\hline Return & Equity & $\begin{array}{l}\text { Security } \\
\text { selection }\end{array}$ & Internal & External & Enhanced & Position & Lending & $\begin{array}{c}\text { Fund } \\
\text { allocation }\end{array}$ & Factors & Environment & Allocation \\
\hline Portfolio & & & & & & & & & & & \\
\hline Mean & 12.11 & 10.89 & 11.74 & 9.03 & 12.31 & 12.30 & 11.82 & 11.70 & 11.68 & 15.88 & 11.70 \\
\hline Std. dev. & 12.32 & 14.21 & 14.84 & 14.24 & 12.01 & 12.00 & 12.11 & 12.20 & 12.18 & 13.54 & 12.13 \\
\hline Sharpe & 0.83 & 0.63 & 0.66 & 0.50 & 0.87 & 0.87 & 0.82 & 0.80 & 0.80 & 1.03 & 0.81 \\
\hline \multicolumn{12}{|l|}{ Benchmark } \\
\hline Mean & 11.75 & 9.47 & 10.76 & 6.42 & 12.12 & 12.17 & 11.75 & 11.75 & 11.72 & 12.05 & 11.75 \\
\hline Std. dev. & 12.11 & 13.54 & 13.97 & 14.10 & 12.02 & 12.00 & 12.11 & 12.11 & 12.13 & 12.50 & 12.11 \\
\hline Sharpe & 0.84 & 0.56 & 0.63 & 0.32 & 0.85 & 0.85 & 0.81 & 0.81 & 0.81 & 0.81 & 0.81 \\
\hline$N$ & 105 & 105 & 105 & 105 & 105 & 105 & 105 & 105 & 105 & 105 & 105 \\
\hline
\end{tabular}

Notes: The table shows the mean and standard deviation of the annualized absolute returns and the Sharpe ratio. Returns are in percent for the Fund's currency basket.

Table 45: Equity-strategies total returns and risk (Fund's currency basket) 


\section{Additional tables: Equity security selection}

- Table 46

- Table 47

- Table 48

\begin{tabular}{lcc|cc|cc}
\hline & \multicolumn{2}{c|}{ Security selection } & \multicolumn{2}{c|}{ Internal } & \multicolumn{2}{c}{ External } \\
\cline { 2 - 7 } & Gross return & Net return & Gross return & Net return & Gross return Net return \\
\hline Mean & 1.42 & 1.27 & 0.98 & 0.93 & 2.60 & 2.15 \\
Std. dev. & 1.50 & 1.50 & 1.82 & 1.82 & 1.97 & 1.96 \\
IR & 0.95 & 0.84 & 0.54 & 0.51 & 1.32 & 1.09 \\
Hit ratio & 0.65 & 0.65 & 0.64 & 0.64 & 0.67 & 0.66 \\
AC(1) & 0.18 & 0.18 & 0.19 & 0.19 & -0.01 & -0.01 \\
Skewness & -0.50 & -0.50 & -0.43 & -0.43 & -0.23 & -0.24 \\
Exc. kurtosis & 0.22 & 0.21 & 0.19 & 0.19 & -0.09 & -0.05 \\
$N$ & 105 & 105 & 105 & 105 & 105 & 105 \\
\hline
\end{tabular}

Notes: The table shows descriptive statistics for the gross and net annualized active returns in percent. IR refers to the mean divided by the standard deviation. Hit ratio is the fraction of months with a positive outcome. $\mathrm{AC}(1)$ refers to the first-order autocorrelation. $N$ is the number of observations in the period. Sample period: $2013-2021$.

Table 46: Equity-security-selection summary statistics of active returns

\begin{tabular}{lcc|cc|cc}
\hline & \multicolumn{2}{c|}{ Security selection } & \multicolumn{2}{c|}{ Internal } & \multicolumn{2}{c}{ External } \\
\cline { 2 - 6 } & Mean & Beta adjustment & Mean & Beta adjustment & Mean & Beta adjustment \\
\hline$\alpha$ & $1.42^{* *}$ & $1.14^{*}$ & 0.98 & 0.56 & $2.60^{* * *}$ & $2.61^{* * *}$ \\
$p$-value & $(0.61)$ & $(0.60)$ & $(0.72)$ & $(0.76)$ & $(0.63)$ & $(0.64)$ \\
$\beta$ & .02 & .06 & .18 & .47 & .0001 & .0001 \\
& & $1.04^{* * *}$ & & $1.05^{* * *}$ & & 1.00 \\
$p$-value & & $(0.01)$ & & $(0.01)$ & $.0 .02)$ \\
$N$ & & .00 & & .00 & 105 & .97 \\
Adjusted $R^{2}$ & 105 & 105 & 105 & 105 & & 0.98 \\
\hline
\end{tabular}

Notes: The table shows the mean annualized active return in the Mean column and the estimated annualized $\alpha$ and $\beta$ from Eq. (1) in the Beta adjustment column. Returns expressed in percent. Newey and West (1987) standard errors in parentheses. $R^{2}$ is the explanatory power from the regression. We denote by stars if $\alpha$ is significantly different from zero and if $\beta$ is significantly different from one. ${ }^{* * * / * * / *}$ indicates significance at the $1 \% / 5 \% / 10 \%$ level.

Table 47: Equity-security-selection gross active returns and gross beta-adjusted active returns 


\begin{tabular}{lc|cc}
\hline & Security selection & Internal & External \\
\hline$\alpha$ & 0.74 & 0.43 & $1.60^{* * *}$ \\
$p$-value & $(0.49)$ & $(0.68)$ & $(0.54)$ \\
$M K T$ & .13 & .53 & .004 \\
& $0.04^{* * *}$ & $0.05^{* * *}$ & 0.02 \\
$p$-value & $(0.01)$ & $(0.01)$ & $(0.02)$ \\
$S M B$ & .0001 & .001 & .15 \\
& $0.05^{* *}$ & 0.04 & $0.07^{*}$ \\
$p$-value & $(0.02)$ & $(0.03)$ & $(0.04)$ \\
$H M L$ & .02 & .23 & .10 \\
& 0.01 & 0.02 & -0.02 \\
$p$-value & $(0.02)$ & $(0.03)$ & $(0.04)$ \\
$R M W$ & .56 & .39 & .54 \\
$p$-value & -0.04 & -0.07 & -0.01 \\
$C M A$ & $(0.04)$ & $(0.04)$ & $(0.06)$ \\
& .27 & .14 & .93 \\
$p$-value & $-0.13^{* * *}$ & $-0.13 * *$ & $-0.10 *$ \\
$N$ & $(0.04)$ & $(0.05)$ & $(0.06)$ \\
Adjusted $R^{2}$ & .003 & .01 & .09 \\
\hline
\end{tabular}

Notes: The table shows the estimated coefficients from the factor model in Eq. (3). $\alpha$ is annualized. Returns expressed in percent. Newey and West (1987) standard errors in parentheses. $N$ refers to the number of observations in the period, and $R^{2}$ is the explanatory power from the regression. We denote by stars if $\alpha$ or the factors are significantly different from zero. ${ }^{* * * / * * / *}$ indicates significance at the $1 \% / 5 \% / 10 \%$ level.

Table 48: Equity-security-selection factor model risk adjustment 


\section{Additional tables: Equity enhanced indexing}

- Table 49

- Table 50

- Table 51

\begin{tabular}{lc|cc}
\hline & $\begin{array}{c}\text { Enhanced } \\
\text { Gross return }\end{array}$ & $\begin{array}{c}\text { Positioning } \\
\text { Gross return }\end{array}$ & $\begin{array}{c}\text { Security. lending } \\
\text { Gross return }\end{array}$ \\
\hline Mean & 0.20 & 0.13 & 0.07 \\
Std. dev. & 0.11 & 0.10 & 0.01 \\
IR & 1.84 & 1.37 & 6.60 \\
Hit ratio & 0.73 & 0.70 & 1.00 \\
AC(1) & 0.25 & 0.24 & 0.54 \\
Skewness & -0.11 & -0.03 & 2.58 \\
Exc. kurtosis & 0.26 & 0.30 & 7.78 \\
$N$ & 105 & 105 & 105 \\
\hline
\end{tabular}

Notes: The table shows descriptive statistics for the gross annualized active returns. Returns expressed in percent. IR refers to the mean divided by the standard deviation. Hit ratio is the fraction of months with a positive outcome. $\mathrm{AC}(1)$ refers to the first-order autocorrelation. $N$ is the number of observations in the period. Sample period: 2013-2021.

Table 49: Equity-enhanced-indexing summary statistics of active returns

\begin{tabular}{lc|cc}
\hline & Enhanced indexing & Asset positioning & Lending \\
\hline$\alpha$ & $0.21^{* * *}$ & $0.13^{* * *}$ & $0.07^{* * *}$ \\
$p$-value & $(0.05)$ & $(0.04)$ & $(0.01)$ \\
$M K T$ & .0001 & .002 & .00 \\
& -0.0001 & 0.0001 & 0.0000 \\
$p$-value & $(0.001)$ & $(0.001)$ & $(0.0001)$ \\
$S M B$ & .89 & .88 & .69 \\
& -0.001 & 0.0003 & -0.0002 \\
$p$-value & $(0.002)$ & $(0.002)$ & $(0.0002)$ \\
$H M L$ & .68 & .88 & .32 \\
$p$-value & 0.0002 & -0.0004 & 0.0001 \\
$R M W$ & $(0.002)$ & $(0.002)$ & $(0.0002)$ \\
& .93 & .83 & .59 \\
$p$-value & 0.004 & 0.004 & -0.0001 \\
$C M A$ & $(0.003)$ & $(0.004)$ & $(0.0003)$ \\
& .26 & .28 & .80 \\
$p$-value & $0.01^{* *}$ & $0.01^{* *}$ & 0.0000 \\
$N$ & $(0.003)$ & $(0.003)$ & $(0.0003)$ \\
Adjusted $R^{2}$ & .02 & .02 & .95 \\
\hline
\end{tabular}

Notes: The table shows the estimated coefficients from the factor model in Eq (3). $\alpha$ is annualized. Returns are expressed in percentage terms. Newey and West (1987) standard errors are provided in parentheses. $R^{2}$ is the explanatory power from the regression and $A R$ is the appraisal ratio. We denote by stars if $\alpha$ or the factors are significantly different from zero. ${ }^{* * * / * * / *}$ indicates significance at the $1 \% / 5 \% / 10 \%$ level.

Table 50: Equity-enhanced-indexing factor model risk adjustment 


\begin{tabular}{lrrr|rr|rr}
\hline & & \multicolumn{2}{c|}{ Enhanced } & \multicolumn{2}{c|}{ Positioning } & \multicolumn{2}{c}{ Lending } \\
\cline { 3 - 8 } Factor & Mean & Est & Prod & Est & Prod & Est & Prod \\
\hline$M K T$ & 11.65 & 0.00 & 0.00 & 0.00 & 0.00 & 0.00 & 0.00 \\
$S M B$ & -0.62 & 0.00 & 0.00 & 0.00 & 0.00 & 0.00 & 0.00 \\
$H M L$ & -4.62 & 0.00 & 0.00 & 0.00 & 0.00 & 0.00 & 0.00 \\
$R M W$ & 3.39 & 0.00 & 0.01 & 0.00 & 0.01 & 0.00 & 0.00 \\
$C M A$ & -2.39 & 0.01 & -0.02 & 0.01 & -0.02 & 0.00 & 0.00 \\
\hline Factor sum & & & -0.01 & & 0.00 & & 0.00 \\
Gross $\alpha$ & & & 0.21 & & 0.13 & & 0.07 \\
Gross mean & & & 0.20 & & 0.13 & & 0.07 \\
\hline
\end{tabular}

Notes: The table shows the factor exposures. In the Est column we show the beta estimates and in the Mean column we show the average return of the factors. In the Prod column, we show how much the Fund has gained or lost from the factor exposure which is the product of the first two columns. Returns are expressed in percentage terms. Sample period: $2013-2021$.

Table 51: Equity-enhanced-indexing factor-exposure analysis 


\section{Additional tables: Equity fund allocation}

- Table 52

- Table 53

\begin{tabular}{lc|ccc}
\hline & Fund allocation & Factors & Environment & Allocation \\
\cline { 2 - 5 } & Gross return & Gross return & Gross return & Gross return \\
\hline Mean & -0.05 & -0.04 & 3.85 & -0.05 \\
Std. dev. & 0.25 & 0.14 & 5.38 & 0.20 \\
IR & -0.18 & -0.25 & 0.72 & -0.24 \\
Hit ratio & 0.49 & 0.45 & 0.60 & 0.51 \\
AC $(1)$ & 0.12 & 0.21 & 0.18 & 0.08 \\
Skewness & -0.11 & -0.66 & 0.29 & -0.14 \\
Exc. kurtosis & -0.03 & 2.84 & 1.65 & 0.64 \\
$N$ & 105 & 105 & 105 & 105 \\
\hline
\end{tabular}

Notes: The table shows descriptive statistics for the gross annualized active returns. Returns expressed in percent. IR refers to the mean divided by the standard deviation. Hit ratio is the fraction of months with a positive outcomes. AC(1) refers to the firstorder autocorrelation. $N$ is the number of observations in the period. Sample period: 2013-2021.

Table 52: Equity-fund-allocation summary statistics of active returns

\begin{tabular}{lc|ccc}
\hline & Fund allocation & Factors & Environment & Allocation \\
\hline$\alpha$ & -0.06 & -0.02 & $3.83^{*}$ & -0.08 \\
$p$-value & $(0.08)$ & $(0.02)$ & $(1.99)$ & $(0.08)$ \\
$M K T$ & .46 & .39 & .06 & .30 \\
& $0.004^{* * *}$ & $0.003^{* * *}$ & 0.07 & 0.001 \\
$p$-value & $(0.001)$ & $(0.001)$ & $(0.04)$ & $(0.001)$ \\
$S M B$ & .003 & .0000 & .14 & .31 \\
& $0.01^{* *}$ & $0.01^{* * *}$ & 0.001 & 0.003 \\
$p$-value & $(0.01)$ & $(0.002)$ & $(0.12)$ & $(0.004)$ \\
$H M L$ & .02 & .00 & 1.00 & .45 \\
$p$-value & $0.01^{* * *}$ & $0.01^{* * *}$ & $-0.35 * * *$ & 0.001 \\
$R M W$ & $(0.004)$ & $(0.001)$ & $(0.13)$ & $(0.003)$ \\
& .01 & .00 & .01 & .85 \\
$p$-value & -0.002 & $0.005^{* *}$ & $-0.59 * * *$ & -0.0004 \\
$C M A$ & $(0.01)$ & $(0.002)$ & $(0.19)$ & $(0.01)$ \\
& .78 & .02 & .002 & .94 \\
$p$-value & $-0.01 *$ & -0.002 & 0.16 & -0.01 \\
$N$ & $(0.01)$ & $(0.002)$ & $(0.24)$ & $(0.01)$ \\
Adjusted $R^{2}$ & .10 & .44 & .51 & .05 \\
\hline
\end{tabular}

Notes: The table shows the estimated coefficients from the factor model in Eq. (3). $\alpha$ is annualized. Returns expressed in percent. Newey and West (1987) standard errors in parentheses. $N$ refers to the number of observations in the period, and $R^{2}$ is the explanatory power from the regression. We denote by stars if $\alpha$ or factors are significantly different from zero. ${ }^{* * * / * * * *}$ indicates significance at the $1 \% / 5 \% / 10 \%$ level.

Table 53: Equity-fund-allocation factor-model risk adjustment 


\section{G Appendix for Section 6}

\section{Additional figures and tables: fixed-income portfolio}

- Table 54

- Figure 29

- Figure 30

\begin{tabular}{lcc|cc}
\hline & \multicolumn{2}{c|}{ Full sample (1998-Sep 2021) } & \multicolumn{2}{c}{ Latest sample (2017-Sep 2021) } \\
\cline { 2 - 5 } & Gross return & Net return & Gross return & Net return \\
\hline Mean & 0.17 & 0.13 & 0.24 & 0.21 \\
Std. dev. & 0.95 & 0.95 & 0.30 & 0.30 \\
IR & 0.18 & 0.13 & 0.18 & 0.13 \\
Hit ratio & 0.61 & 0.59 & 0.63 & 0.61 \\
AC(1) & 0.67 & 0.67 & 0.14 & 0.14 \\
Skewness & -0.35 & -0.37 & -0.31 & -0.31 \\
Exc. kurtosis & 18.96 & 18.98 & 1.87 & 1.87 \\
$N$ & 285 & 285 & 57 & 57 \\
\hline
\end{tabular}

Notes: The table shows descriptive statistics for the gross annualized active returns in percent. IR refers to the mean divided by the standard deviation. Hit ratio is the fraction of months with a positive outcome. $\mathrm{AC}(1)$ refers to the first-order autocorrelation. $N$ the number of observations in the period.

Table 54: Fixed-income summary statistics of active returns

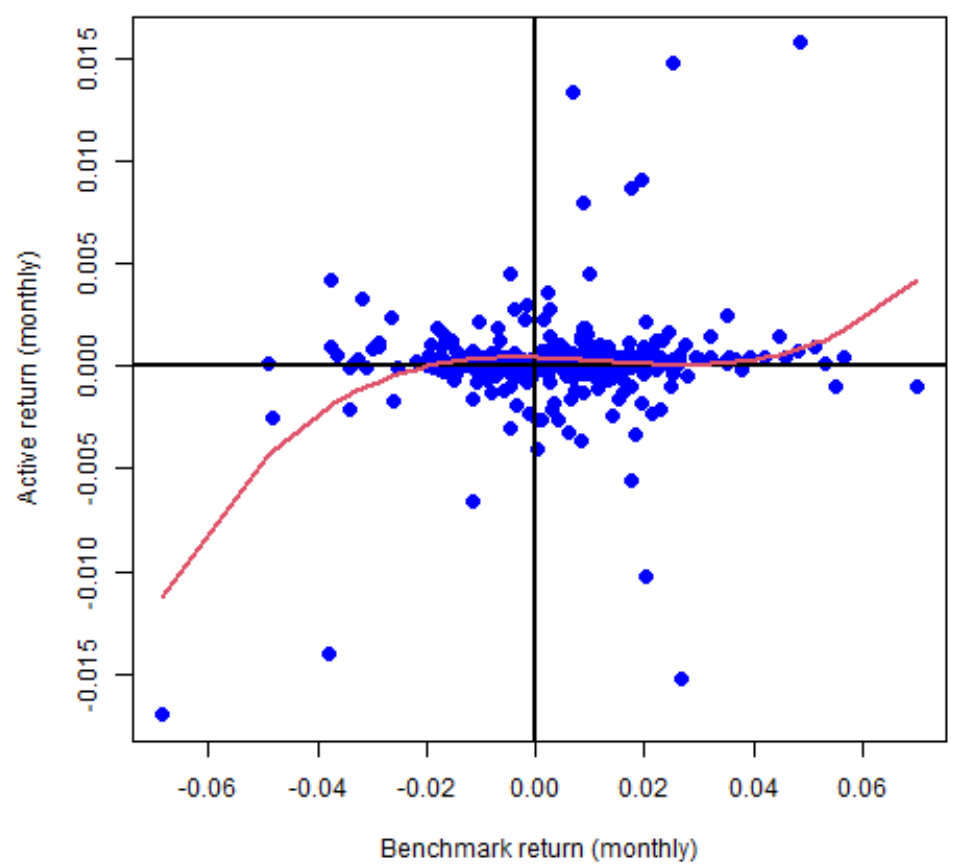

Figure 29: Fixed-income active returns against benchmark returns 


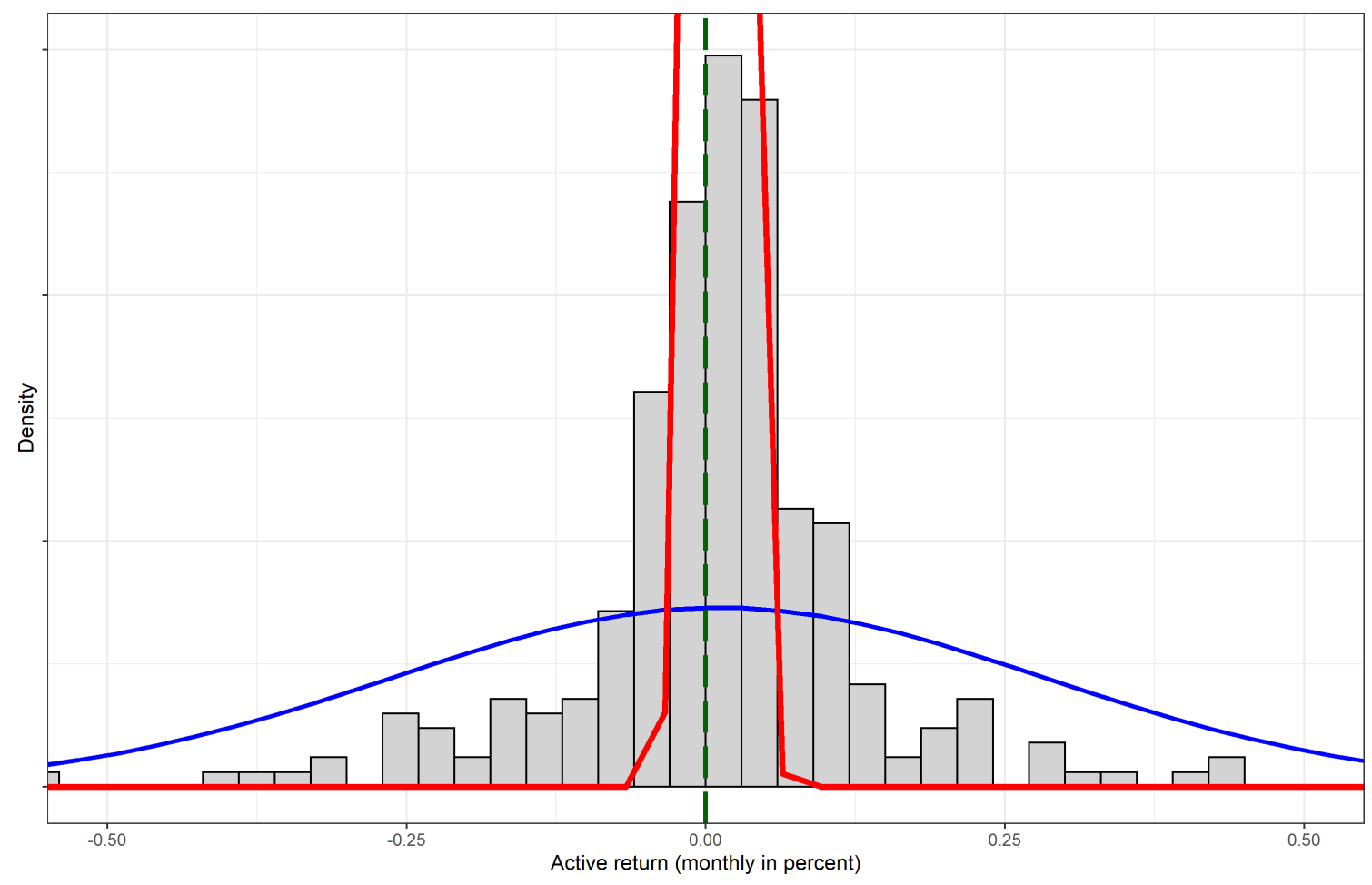

Figure 30: Histogram of the fixed-income net active returns 
H Appendix for Section 7

Additional figures and tables: Fixed-income subportfolios

- Table 55

- Figure 31

\begin{tabular}{|c|c|c|c|c|c|c|c|c|c|}
\hline Return & Fixed & $\begin{array}{l}\text { Security } \\
\text { selection }\end{array}$ & Internal & Enhanced & Position & Lending & $\begin{array}{c}\text { Fund } \\
\text { allocation }\end{array}$ & Environment & Allocation \\
\hline Portfolio & & & & & & & & & \\
\hline Mean & 2.03 & 3.80 & 3.80 & 1.64 & 1.61 & 3.44 & 1.78 & 3.97 & 1.78 \\
\hline Std. dev. & 4.80 & 5.30 & 5.30 & 4.76 & 4.76 & 5.05 & 4.81 & 6.71 & 4.81 \\
\hline Sharpe & 0.29 & 0.60 & 0.60 & 0.22 & 0.21 & 0.56 & 0.24 & 0.50 & 0.24 \\
\hline Benchmark & & & & & & & & & \\
\hline Mean & 1.96 & 3.39 & 3.39 & 1.36 & 1.36 & 3.41 & 1.96 & 3.68 & 1.96 \\
\hline Std. dev. & 4.86 & 5.62 & 5.62 & 4.73 & 4.73 & 5.05 & 4.86 & 6.77 & 4.86 \\
\hline Sharpe & 0.29 & 0.49 & 0.49 & 0.16 & 0.16 & 0.55 & 0.28 & 0.45 & 0.28 \\
\hline$N$ & 105 & 84 & 84 & 105 & 105 & 71 & 105 & 54 & 105 \\
\hline
\end{tabular}

Notes: The table shows the mean and standard deviation of the annualized total returns and the Sharpe ratio. Returns expressed in percent.

Table 55: Fixed-income strategies total return and risk

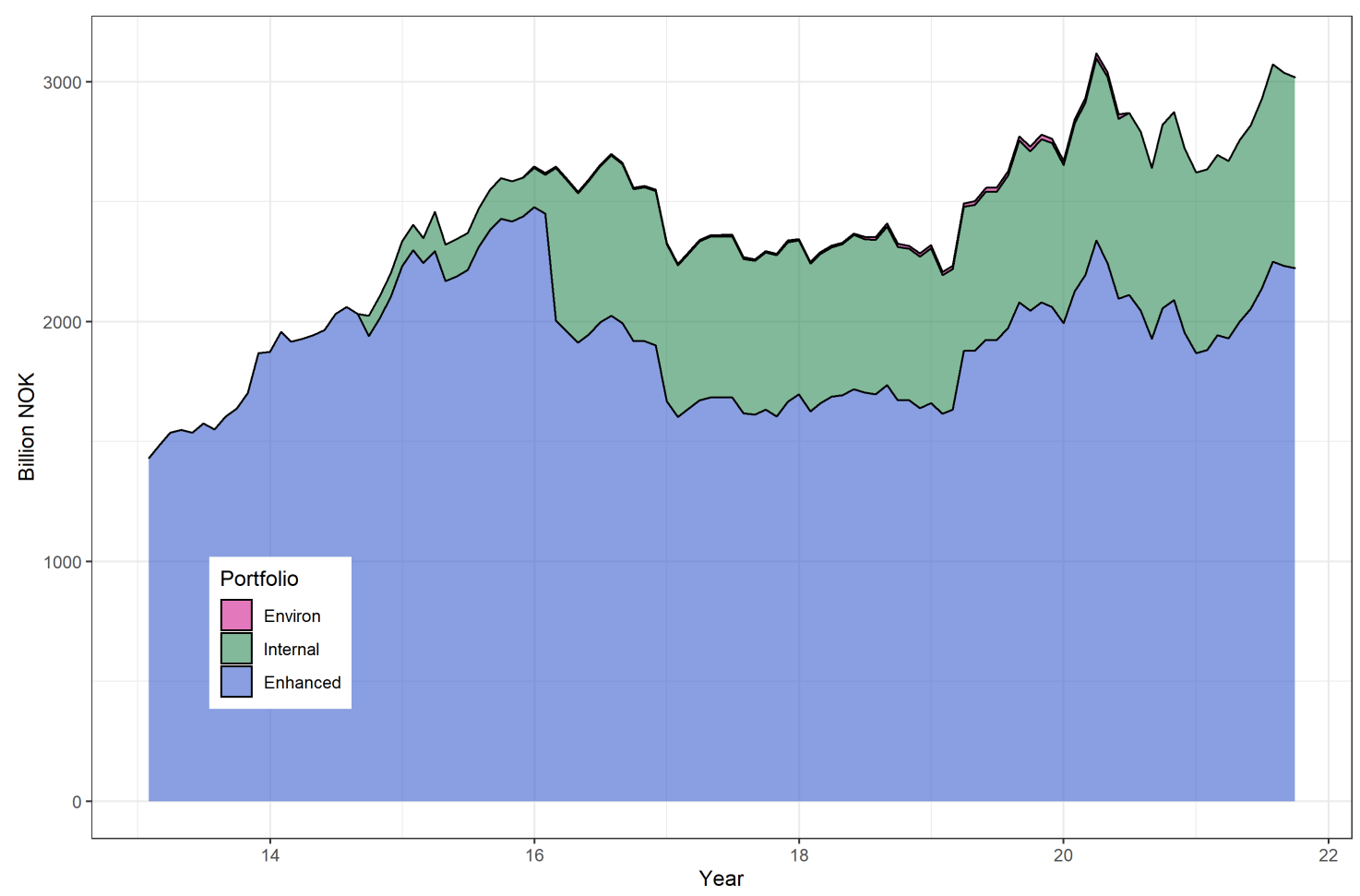

Figure 31: Fixed-income strategies AUM 
Additional figures and tables: Fixed-income security selection

- Table 56

- Table 57

\begin{tabular}{lcc}
\hline & \multicolumn{2}{c}{ Internal security selection } \\
\cline { 2 - 3 } & Gross return & Net return \\
\hline Mean & 0.41 & 0.36 \\
Std. dev. & 0.88 & 0.88 \\
IR & 0.47 & 0.41 \\
Hit ratio & 0.46 & 0.43 \\
AC(1) & 0.03 & 0.03 \\
Skewness & 2.62 & 2.61 \\
Exc. kurtosis & 11.51 & 11.52 \\
$N$ & 84 & 84 \\
\hline
\end{tabular}

Notes: The table shows descriptive statistics for the gross annualized active returns in percent. IR refers to the mean divided by the standard deviation. Hit ratio is the fraction of months with a positive outcome. AC(1) refers to the first-order autocorrelation. $N$ is the number of observations in the period. Sample period: $2014-2021$.

Table 56: Fixed-income-security-selection summary statistics of active returns

\begin{tabular}{lc}
\hline & Internal security selection \\
\hline$\alpha$ & $0.55^{* *}$ \\
& $(0.26)$ \\
-value & .04 \\
$T E R M$ & 0.01 \\
$p$-value & $(0.01)$ \\
$D E F$ & .12 \\
& $-0.07^{* * *}$ \\
$p$-value & $(0.01)$ \\
$N$ & .00 \\
Adjusted $R^{2}$ & 84 \\
\hline
\end{tabular}

Notes: The table shows the estimated coefficients from the factor model in Eq. (4). $\alpha$ is annualized. Returns expressed in percent. Newey and West (1987) standard errors in parentheses. $N$ refers to the number of observations in the pe$\operatorname{riod}$, and $R^{2}$ is the explanatory power from the regression. We denote by stars if $\alpha$ or the factors are significantly different from zero. ${ }^{* * * / * * / *}$ indicates significance at the 1\%/5\%/10\% level.

Table 57: Fixed-income-security-selection factor model risk adjustment 


\section{Additional figures and tables: fixed-income enhanced indexing}

The sample period for the fixed-income security lending begins in November 2015.

- Table 58

- Table 59

- Table 60

\begin{tabular}{lc|cc}
\hline & Enhanced & Positioning & Security lending \\
\cline { 2 - 4 } & Gross return & Gross return & Gross return \\
\hline Mean & 0.28 & 0.25 & 0.03 \\
Std. dev. & 0.16 & 0.16 & 0.01 \\
IR & 1.79 & 1.57 & 6.03 \\
Hit ratio & 0.74 & 0.73 & 0.99 \\
AC(1) & 0.00 & 0.00 & 0.76 \\
Skewness & -0.45 & -0.35 & 0.22 \\
Exc. kurtosis & 3.08 & 3.16 & -0.82 \\
$N$ & 105 & 105 & 71 \\
\hline
\end{tabular}

Notes: The table shows descriptive statistics for the gross annualized active returns in percent. IR refers to the mean divided by the standard deviation. Hit ratio is the fraction of months with a positive outcome. AC(1) refers to the first-order autocorrelation. $N$ is the number of observations in the period. Sample period: 2013-2021, except for security lending (2015-2021).

Table 58: Fixed-income-enhanced-indexing summary statistics of active returns

\begin{tabular}{lc|cc}
\hline & Enhanced & Positioning & Lending \\
\hline$\alpha$ & $0.28^{* * *}$ & $0.24^{* * *}$ & $0.03^{* * *}$ \\
& $(0.05)$ & $(0.05)$ & $(0.01)$ \\
$p$-value & .00 & .0000 & .0000 \\
$T E R M$ & $-0.01^{* *}$ & $-0.01^{* *}$ & 0.0000 \\
& $(0.003)$ & $(0.003)$ & $(0.0001)$ \\
$p$-value & .03 & .03 & .79 \\
$D E F$ & $0.01^{*}$ & $0.01^{*}$ & 0.0000 \\
$p$-value & $(0.004)$ & $(0.004)$ & $(0.0001)$ \\
$N$ & .08 & .07 & .90 \\
Adjusted $R^{2}$ & 105 & 105 & 71 \\
\hline
\end{tabular}

Notes: The table shows the estimated coefficients from the factor model in Eq. (4). $\alpha$ is annualized. Returns expressed in percent. Newey and West (1987) standard errors in parentheses. $N$ refers to the number of observations in the period, and $R^{2}$ is the explanatory power from the regression. We denote by stars if $\alpha$ or the factors are significantly different from zero. ${ }^{* * * / * * / *}$ indicates significance at the $1 \% / 5 \% / 10 \%$ level.

Table 59: Fixed-income-enhanced-indexing factor model risk adjustment 


\begin{tabular}{lrrrrr|rr}
\hline & & \multicolumn{2}{c|}{ Enhanced } & \multicolumn{2}{c|}{ Positioning } & \multicolumn{2}{c}{ Lending } \\
\cline { 3 - 8 } Factor & Mean & Est & Prod & \multicolumn{1}{c}{ Est } & Prod & Est & Prod \\
\hline TERM & 3.58 & -0.01 & -0.03 & -0.01 & -0.02 & 0.00 & 0.00 \\
$D E F$ & 3.58 & 0.01 & 0.03 & 0.01 & 0.03 & 0.00 & 0.00 \\
\hline Factor sum & & & 0.00 & & 0.00 & & 0.00 \\
Gross $\alpha$ & & & 0.28 & & 0.24 & & 0.03 \\
Gross mean & & & 0.28 & & 0.25 & & 0.03 \\
\hline
\end{tabular}

Notes: The table shows the factor exposures. The Est column shows the beta estimates, and the Mean column shows the average return of the factors. The Prod column shows how much the Fund has gained or lost from the factor exposure, which is the product of the first two columns. Returns expressed in percent.

Table 60: Fixed-income-enhanced-indexing factor-exposure analysis 


\section{Additional figures and tables: fixed-income fund allocation}

The sample period for the environmental strategy covers the period January 2016 to June 2020.

- Table 61

- Table 62

\begin{tabular}{lc|cc}
\hline & Fund allocation & Environment & Allocation \\
\cline { 2 - 4 } & Gross return & Gross return & Gross return \\
\hline Mean & -0.18 & 0.28 & -0.18 \\
Std. dev. & 0.38 & 0.51 & 0.38 \\
IR & -0.47 & 0.56 & -0.47 \\
Hit ratio & 0.50 & 0.59 & 0.50 \\
AC $(1)$ & 0.05 & -0.14 & 0.05 \\
Skewness & -0.47 & -0.17 & -0.47 \\
Exc. kurtosis & 0.67 & 1.50 & 0.67 \\
$N$ & 105 & 54 & 105 \\
\hline
\end{tabular}

Notes: The table shows descriptive statistics for the gross annualized active returns in percent. IR refers to the mean divided by the standard deviation. Hit ratio is the fraction of months with a positive outcome. $\mathrm{AC}(1)$ refers to the first-order autocorrelation. $N$ is the number of observations in the period. Sample period: 2013-2021.

Table 61: Fixed-income-fund-allocation summary statistics of active returns

\begin{tabular}{lc|cc}
\hline & Fund allocation & Environmental & Allocation \\
\hline$\alpha$ & -0.12 & 0.25 & -0.12 \\
& $(0.12)$ & $(0.18)$ & $(0.12)$ \\
$p$-value & .34 & .17 & .34 \\
$T E R M$ & $-0.03^{* * *}$ & 0.0004 & $-0.03^{* * *}$ \\
& $(0.01)$ & $(0.01)$ & $(0.01)$ \\
$p$-value & .0001 & .98 & .0001 \\
$D E F$ & $0.01^{* * *}$ & 0.01 & $0.01^{* * *}$ \\
& $(0.003)$ & $(0.01)$ & $(0.003)$ \\
$p$-value & .002 & .15 & .003 \\
$N$ & 105 & 54 & 105 \\
Adjusted $R^{2}$ & 0.23 & -0.02 & 0.23 \\
\hline
\end{tabular}

Notes: The table shows the estimated coefficients from the factor model in Eq. (4). $\alpha$ is annualized. Returns expressed in percent. Newey and West (1987) standard errors in parentheses. $N$ refers to the number of observations in the period, and $R^{2}$ is the explanatory power from the regression. We denote by stars if $\alpha$ or the factors are significantly different from zero. ${ }^{* * * / * * / *}$ indicates significance at the $1 \% / 5 \% / 10 \%$ level.

Table 62: Fixed-income-fund-allocation factor model risk adjustment 


\section{Appendix for Section 8}

\section{Additional figures and tables: Real-estate portfolio}

- Figure 32

- Table 63

- Table 64

- Table 65

- Figure 33

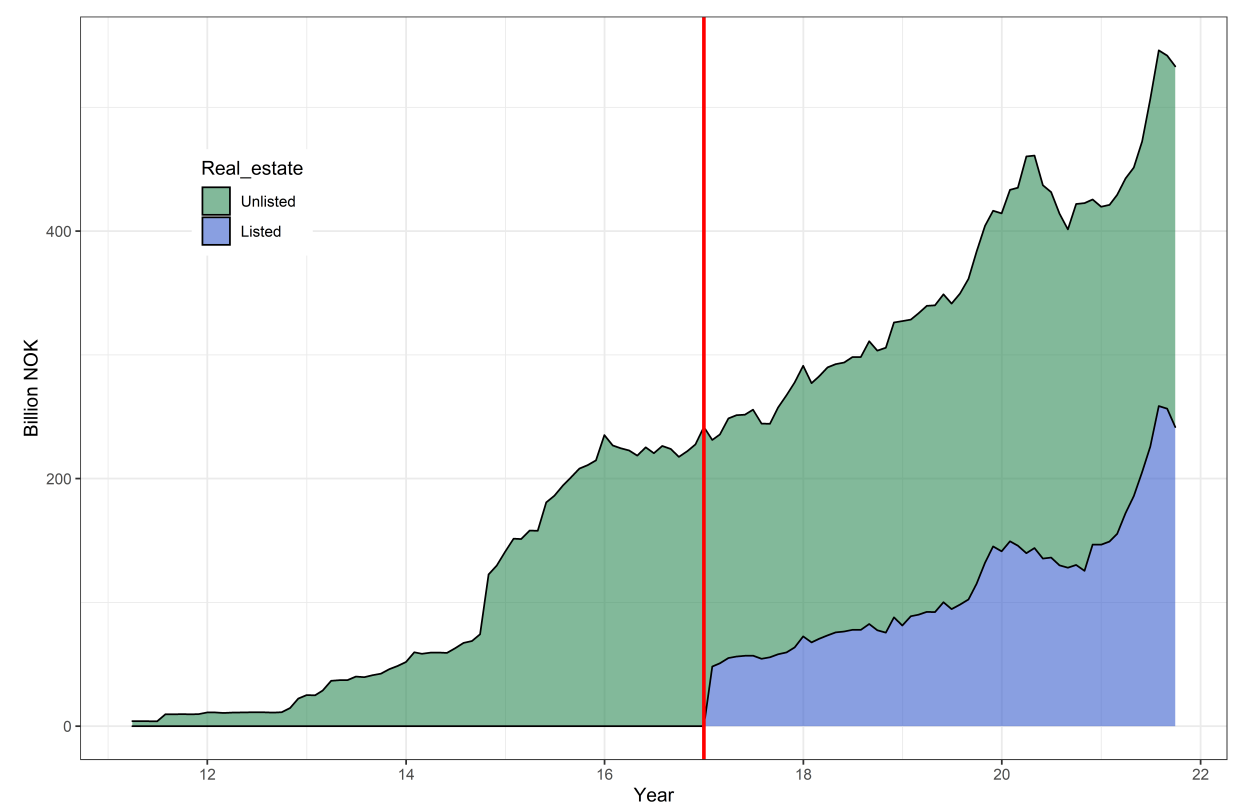

AUM (in billion NOK) in real estate. Red vertical line indicates new financing model from 2017.

Figure 32: Real-estate AUM

\begin{tabular}{lrcrrrc}
\hline & $\begin{array}{c}\text { Portfolio } \\
\text { gross }\end{array}$ & Benchmark & Gross & & & \\
& 6.62 & 8.11 & -1.50 & -1.61 & -1.63 & -1.72 \\
\hline Mean & 7.67 & 8.28 & 5.04 & 5.03 & & \\
Std. dev./TE & 0.72 & 0.85 & -0.30 & -0.32 & & \\
Sharpe ratio/IR & & & 3.23 & 3.22 & & \\
Standard error & & & 0.64 & 0.62 & & \\
$p$-value & 19 & 19 & 19 & 19 & 19 & 19 \\
$N$ & & & &
\end{tabular}

Notes: The table shows statistics for the annualized returns and quarterly gross value added. Quarterly returns are annualized by multiplying by 4 . Returns expressed in percent. Mean value added is in quarterly NOK billion. $N$ refers to the number of observations in the period. Sample period 2017-2021.

Table 63: Total-real-estate summary statistics 


\begin{tabular}{lccrccc}
\hline & $\begin{array}{c}\text { Portfolio } \\
\text { gross }\end{array}$ & Benchmark & $\begin{array}{r}\text { Gross } \\
\text { active }\end{array}$ & Net active & Gross VA & Net VA \\
\hline Mean & 7.04 & 7.17 & -0.13 & -0.29 & -0.32 & -0.42 \\
Std. dev./TE & 3.90 & 6.97 & 6.22 & 6.21 & & \\
Sharpe ratio/IR & 1.53 & 0.87 & -0.02 & -0.05 & & \\
Standard error & & & 2.44 & 2.43 & & \\
$p$-value & & & 0.96 & 0.91 & & \\
$N$ & 19 & 19 & 19 & 19 & 19 & 19 \\
\hline
\end{tabular}

Notes: The table shows statistics for the annualized returns and quarterly gross value added. Quarterly returns (\%) are annualized by multiplying by 4 . Mean value added is in quarterly NOK billion. Newey and West (1987) standard errors. $N$ refers to the number of observations in the period. Sample period: $2017-2021$.

Table 64: Unlisted-real-estate summary statistics

\begin{tabular}{|c|c|c|c|c|}
\hline & $\begin{array}{l}\text { Portfolio } \\
\text { gross }\end{array}$ & Benchmark & $\begin{array}{l}\text { Gross } \\
\text { active }\end{array}$ & Gross VA \\
\hline Mean & 6.53 & 10.35 & -3.81 & -0.46 \\
\hline Std. dev./TE & 18.66 & 9.77 & 12.29 & \\
\hline Sharpe ratio/IR & 0.29 & 0.95 & -0.31 & \\
\hline Standard error & & & 5.08 & \\
\hline$p$-value & & & 0.45 & \\
\hline$N$ & 57 & 57 & 57 & 57 \\
\hline
\end{tabular}

Notes: The table shows statistics for the annualized returns and monthly gross value added. Returns expressed in percent. Value added is in NOK billion. Newey and West (1987) standard errors. $N$ refers to the number of observations in the period. Sample period: $2017-2021$.

Table 65: Listed-real-estate summary statistics

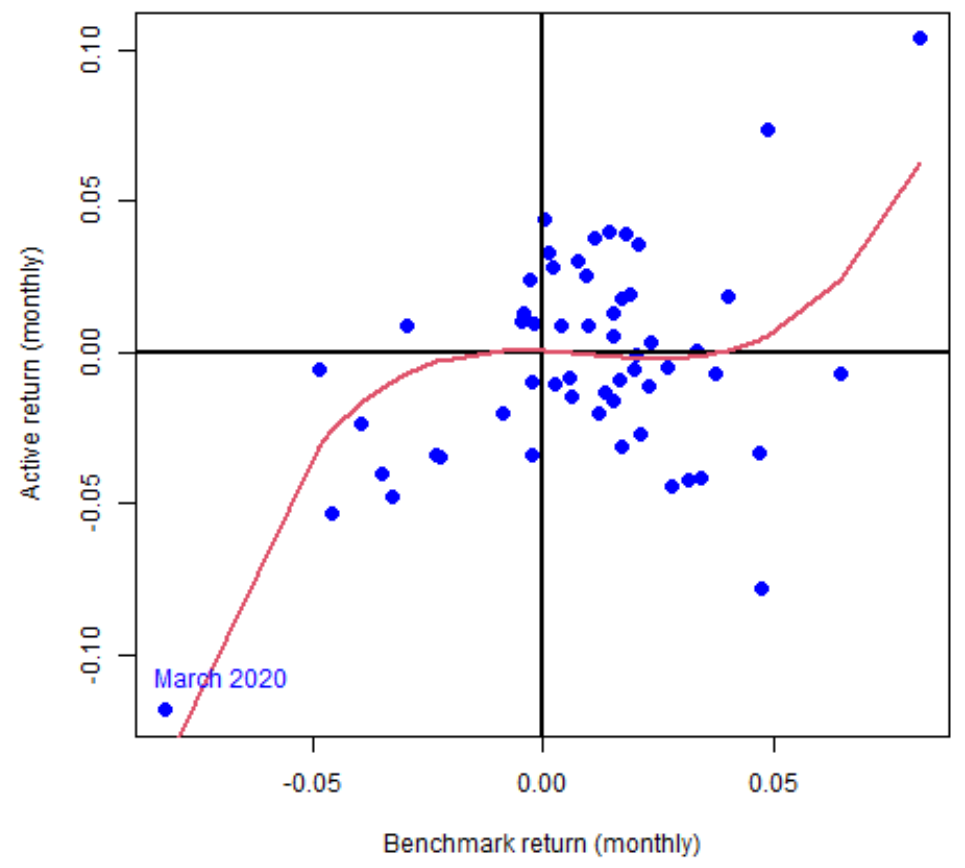

Figure 33: Listed-real-estate active returns against benchmark returns 